NBS MONOGRAPH 66 I.S. G.A.

Electron Optical Studies

Of Low-Pressure Gases AECEIYED

AUG 3003

U. S. DEPRTRE:

GASTERM RESTEZ

LBBDRATARY A.R.

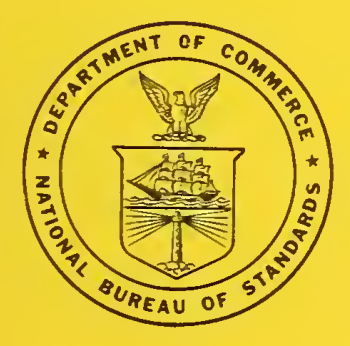

U.S. DEPARTMENT OF COMMERCE

NATIONAL BUREAU OF STANDARDS 


\section{THE NATIONAL BUREAU OF STANDARDS}

\section{Functions and Activities}

The functions of the National Bureau of Standards are set forth in the Act of Congress, March 3, 1901, as amended by Congress in Public Law 619, 1950. These include the development and maintenance of the national standards of measurement and the provision of means and methods for making measurements consistent with these standards; the determination of physical constants and properties of materials; the development of methods and instruments for testing materials, devices, and structures; advisory services to government agencies on scientific and technical problems; invention and development of devices to serve special needs of the Government; and the development of standard practices, codes, and specifications. The work includes basic and applied research, development, engineering, instrumentation, testing, evaluation, calibration services, and various consultation and information services. Research projects are also performed for other government agencies when the work relates to and supplements the basic program of the Bureau or when the Bureau's unique competence is required. The scope of activities is suggested by the listing of divisions and sections on the inside of the back cover.

\section{Publications}

The results of the Bureau's research are published either in the Bureau's own series of publications or in the journals of professional and scientific societies. The Bureau itself publishes three periodicals available from the Government Printing Office: The Journal of Research, published in four separate sections, presents complete scientific and technical papers; the Technical News Bulletin presents summary and preliminary reports on work in progress; and Basic Radio Propagation Predictions provides data for determining the best frequencies to use for radio communications throughout the world. There are also five series of nonperiodical publications: Monographs, Applied Mathematics Series, Handbooks, Miscellaneous Publications, and Technical Notes.

A complete listing of the Bureau's publications can be found in National Bureau of Standards Circular 460, Publications of the National Bureau of Standards, 1901 to June 1947 (\$1.25), and the Supplement to National Bureau of Standards Circular 460, July 1947 to June 1957 (\$1.50), and Miscellaneous Publication 240, July 1957 to June 1960 (includes Titles of Papers Published in Outside Journals 1950 to 1959) (\$2.25); available from the Superintendent of Documents, Government Printing Office, Washington, D.C. 20402 


\section{Electron Optical Studies of Low-Pressure Gases}

L. Marton, David C. Schubert, and S. R. Mielczarek

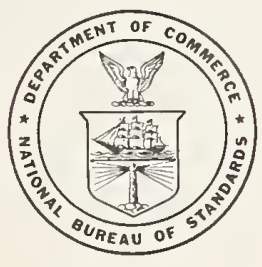

National Bureau of Standards Monograph 66 Issued August 16, 1963 
Library of Congress Catalog Card Number: 63-60061 


\section{Preface}

This project had its origin in a proposal which Dr. L. Marton, the principal inrestigator on the project, made to the Office of Naral Research in June 1954. The proposal was based on earlier experiments on electron optical schlieren methods which had been carried out under Dr. Marton's direction in the Electron Physics Section of the National Bureau of Standards.

Realizing the importance of this net approach to lotr-pressure research, the Office of Naral Research actirated the project on February 1, 1955. Since that time, a number of people besides the principal inrestigator hare worked on the project. Chief among these rere Dr. Darid C. Schubert, who serred as project leader throughout the history of the project, and Stanley R. Mielczarek. Space does not permit mention of all the other members of the Electron Physics Section who assisted in the work from time to time, but recognition must be giren to the contribution of John Kneisly III, who designed the Cassegrainian system used to provide the synchronizing light pulses in the stroboscopic operation. In addition, we thank August B. Dauses and Lytle E. Mann of the Electron Physics Section instrument shop, whose careful, patient work made the experimental part of the program possible. Our thanks are also due to the rarious personnel of the Office of Naral Research and especially to Ralph Cooper who serred as Contract Officer during inost of the life of the project and whose interest in the project and understanding of the difficulties inrolved made the task much more pleasant than it might otherwise hare been.

John A. Simpson, Chief

Electron Physics Section 


\section{Contents}

Preface

1. The importance and significance of low-pressure gas studies

2. Existing techniques for mapping low-pressure gas flow

3. The principles of electron optical schlieren

4. Realization of the electron optical schlieren method

4.1. Electron optics

a. Optics of the beam-generating section

b. Optics of the test section......

c. Optics of the detection section.-.-

5. Gas kinetics

5.1. Beam source

5.2. Chopped beams.

6. Stroboscopic mechanisms _...

6.1. Optics

6.2. Electronics.

7. High-vacuum section
Page

1

2

3

6

6

6

8

11

12

13

14

14

16

16

8. Intermediate-vacuum section

Page

9. Vacuum pump system

10. Auxiliary equipment

11. Experimental results 21

12. Interpretation of the data_._._._._._. 21

12.1. Scanning of the negatives_........ 22

12.2. Computation of velocity distributions _ 24

12.3. Reliability of the iteration process_-_- 25

13. Appendix

13.1. The photographic camera_._._._._. 27

13.2. Earth's field neutralization_________ 29

13.3. Variation of the compensating field with position along the $z$-axis

13.4. Relative positions of the chopper disks

13.5. Computation of the initial distribution function .........................

13.6. Simplified approximate computation of the molecular density distribution --

14. References 


\title{
Electron Optical Studies of Low-Pressure Gases
}

\author{
L. Marton, David C. Schubert, and S. R. Mielczarek
}

\begin{abstract}
This document is the final report of research carried on in the Electron Physies Section of the National Bureau of Standards during the period from February 1, 1955 to March of 1962 in developing an electron optical method for the visualization of low-pressure gas flow. The goal of this work was to develop and demonstrate the suitability of electron optical techniques for recording the spatial distribution of gas molecules at fixed times with sufficient accuracy that the velocity distribution could be derived. The work concentrated on the properties of ehopped molecular beams of cadmium at extremely low pressures where the densities of the particles in the molecular beam pulse correspond to a pressure of less than 10-7 Torr. The project was successful. During the course of this program, equipment was built, methods of operation were developed, the theory of the electron optical schlieren was developed in some detail, and ways of efficiently converting the data obtained into velocity distributions of the gas molecule were investigated.
\end{abstract}

\section{The Importance and Significance of Low-Pressure Gas Studies}

Low-pressure gas studies are concerned with the motions of gas molecules under conditions such that the mean-free paths of the molecules are significantly larger than the mean interaction distances, and therefore the equations of continuum aerodynamics are no longer applicable. In the limit of approaching zero pressure, gas molecules behave entirely independently $[1,2],{ }^{1}$ moving in straight lines except when they strike solid or liquid surfaces where they may be absorbed or reflected and may exchange momentum and energy with the surface.

Early in the history of the gas flow problem, researchers assumed that molecules were reflected from a solid surface with an angular distribution proportional to the cosine of the angle between the reflected beam and the normal to the surface [3], and that the velocity distribution corresponded to the temperature of the reflecting surface [4]. Measurements of heat transfer to surfaces, however, showed evidence that the temperature of gas molecules lay somewhere between the temperature of the incident gas atoms and that of the surface. On this empirical basis, the concept of the accommodation coefficient $\alpha$ was defined as the ratio between the effective temperature change imposed upon the gas molecules striking the surface and the initial temperature difference between the solid surface and the gas [5]. Thus,

$$
\alpha_{T}=\frac{T_{r}-T_{i}}{T_{s}-T_{i}}
$$

where $T_{i}, T_{r}$, and $T_{s}$ are the temperatures of the incident beam, the reflected beam, and the surface, respectively. When the so-called cosine law distribution for reflected gas molecules was used to predict the tangential forces on the boundary surfaces in gas viscosity experiments, the predicted forces consistently exceeded the measured forces. To reconcile theory with experiment, it was assumed that the reflected molecules retain a portion of their mean incident momentum components tangent to the surface. This empirical

1 Figures in brackets indicate the literature references at the end of this Monograph. property is expressed quantitatively by the viscous slip coefficient, defined as

$$
\eta=\bar{P}_{r} / \bar{P}_{i}
$$

where $\bar{P}_{i}$ and $\bar{P}_{r}$ are the average components of particle momentum tangent to the surface before and after reflection.

These are the relationships which should be applied to the boundary conditions of ordinary problems in continuum aerodynamics. One can expect that both the viscous slip and accommodation coefficients will be functions of the particular gas, the surface material, and the condition of the surface. Unfortunately, existing data concerning these coefficients are rather scanty and somewhat conflicting [6]. Moreover, calculations of microscopic interactions of atoms impinging on surfaces are still incomplete $[7,8]$.

Without dependable knowledge of the boundary conditions on model surfaces in a wind tunnel, one may expect that difficulty arises in relating accurately the observed wind tunnel phenomena to the basic physical properties of the flow. Determination of these coefficients for a single substance suitable for use in a wind tunnel would be considered a major breakthrough [9]. These coefficients are readily computed from velocity distributions of reflected molecules before and after impact with a solid surface. Availability of experimental velocity distribution data for reflected atoms would also be a powerful tool fo: investigating the physics of surfare interactions, which are now incompletely understood.

In the interaction of the upper atmosphere witi missiles and satellites (a field of study which came into prominence during the course of this project), conditions approaching the limit of low-density How exist. The mean-free path of the molecules becomes large in comparison with the dimensions of the vehicle. Consequently, continuum effects are absent and transfer of energy and momentum between the gas and the vehicle reduces to singleparticle interaction. The computation of heating and drag effects at high altitudes thus depends decisively upon knowledge of molecular velocity distributions after reflection [10]. 
Besides these two more obvious uses for such velocily field chata, there are useful applications in confinum aerodynanics, for at a short distance from a sharp leading edge in hypersonic flow the rarefied state may occur locally, although the greater part of the gas stream is much more dense [11]. Whether conditions such as these are of interest from the pure phrsic's standpoint or for the n101* applied needs of aerodynamies does not matter. There exists a great need for methods of determining precisely the velocity distribution of gas atoms. The problem of obtaining these data interested a large number of investigators in the past and undoubtedly will continue to do so in the future. It is probably worthwbile at this point to review some of the methods that have been applied in the past and to show where they fail to give the necessary precision or sensitivity. One nuay hope-by means of the electron optical schlieren techinique-to obtain more dependable clata which are valid in the lowest pressure regions.

\section{Existing Techniques for Mapping Low-Pressure Gas Flow}

The methods of mapping gas flow are naturally divided into two groups, those useful at gas pressures of around $\mu$ atm and those that have been dereloped for pressures below $\mu$. In some cases the same method has been extended by careful work to cover both regions.

One method, highly developed recently, for work at around atmospheric pressure is the hot wire detector $[12,13]$. In this technique the hot wire detector measures the temperature of a wire with known geometry and heating power supported in the gas stream. The gas stream cools the wire, which in a manner similar to a Pirani thermocouple vacuum gage gives evidence as to the conditions of flow at that point. Unfortunately, the response is really that of a flowmeter. It measures a combination of pressure and velocity. With careful design, the response can be made surprisingly rapid. Fluctuation frequencies up to $10^{5} / \mathrm{sec}$ have been measured in this way at atmospheric pressure [14]. At low pressures, however, the response is correspondingly more sluggish, and it is thus unsuited to velocity measurements at low pressures. It has been highly developed over the years and remains the standard wind tunnel tool of today.

In the higher pressure range, perhaps the most elegant, most used, and most accurate method of mapping gas flow is the optical interferometer [15]. In this case, light is passed through the gas; differences in optical path length show up as light and dark fringes in an interferometric display. The space between each two successive dark fringes corresponds to one wavelength in path difference. This method maps an entire flow in two dimensions, since the optical path length is a function of the integral of density along the path. It is not useful in the case of a truly three-dimensional flow, but then only point-by-point techniques are. Moreover, being a continuum device, it fails from lack of sensitivity when the refractive inclex of the rarefied gas becomes indistinguishable from that of free space. At the present time, with the present equipment, the interferometer is too insensitive for studies of rarefied gases. However, in the range where it is useful it remains perhaps the best of all the current methods.

Another optical method which shares many of the advantages and the disadvantages of the optical interferometer is that of optical schlie- ren [16]. This method also makes use of the continuum properties of the gas. A light beam is passed through the flow and observed in such a manner that the prismatic effects of gradient of density are observed. The word "schlieren" is a German expression meaning "striation," and it describes the early application of the method to observe striations in the flow patterns of heated gases. The fact that the density gradients (rather than the density itself') are observed has both advantages and disadvantages. For sharply changing flows the gradient of density is somewhat easier to detect than the density itself but, on the other hand, if one is interested in mapping the entire flow, the gradient is one step further from the desired results than in the case of the interferometric method. Although highly developed, the optical schlieren method fails in the low-density range, as does the optical interferometer, because the index of refraction approaches unity throughout the region of gas flow. This method, however, does serve as the inspiration for the electron optical schlieren method and the theory will be developed in some detail in a later section.

In view of the importance of the problem, it is not surprising that many efforts have been made to develop techniques which are workable at much lower pressures. One general method, which although tedious and awkward in application is frequently used, is the capture of individual molecules or groups of molecules as they arrive at a target normal to the direction of flow. Several variations have been tried, all of them with some success. First in this class is the condensation target $[1,17]$. The beam material is collected on a target which may be at a low temperature or react chemically with the atomic species. With careful development techniques $[18,19]$, or Geiger counters, and radioactive tracing [20,21], it is possible to get extremely high sensitivity, detecting the arrival of only a few or of a few thousand atoms. It is a slow, cumbersome method not much used and certainly not easily adaptable for velocity determination.

The arrival of atoms can also be detected by the surface ionization gage $[22,23]$. This technique makes use of the fact that atoms striking a hot tungstelı wire may be ionized if any of the work functions of the tungsten exceed the ionization potential of the atom. Using tungsten, it is 
possible to get nearly 100 percent efficiency of detection for the alkali metals. This method can be adapted to give time resolution and will serve as a point-by-point detector to map a field of flow. Again, it is a point-by-point detector, and hence it is a time-consuming, difficult problem to map a flow of any extent. But it can be and has been done.

A detector which offers some promise is the so-called electron bombardment detector [24]. In this case, the molecular beam is bombarded with electrons which ionize about one atom in $10^{4}$. The ions pass through a mass spectrograph, and in this manner the effect of most background gases can be removed. With the use of time-resolved mass spectrometry, this method could also give time-resolved information. However, again the difficulties inherent in such a method make it seem rather unattractive.

A more obvious and straightforward approach is to adapt the techniques used in continuum aerodynamics to the low-pressure region by using radiation which interacts more strongly with the gas atoms. In this effort, the absorption of ultraviolet light and of X-radiation has been used [25, 26]. At great expense and great effort, this method can be made to extend the range of the usual optical techniques several orders of magnitude lower. This technique requires, however, the use of either reflecting or quartz transmission optics, and in the long run seems to be rather limited.

Another technique which has been tried makes use of the afterglow [27] of nitrogen (following RF excitation at a point upstream from the test area). This afterglow is sufficiently bright to be detected and gives a response proportional to the gas density. This ingenious technique suffers from the fact that now one is dealing with an excited and partially ionized gas whose properties differ from those of the neutral beams one started out to study. In many cases the focus of interest is on just those properties which are different in the neutral molecule from what they are in an excited molecule or ion, and so again this technique has its limitations.

In the search for radiation which interacts more strongly with the gas atoms, the interaction of electrons with atoms was not overlooked. The direct application of this effect yields the method which is known as attenuation by scattering [28]. This method, which has been used to measure the pressure variations behind shock waves, employs a thin, pencil-shaped electron beam which passes through the gas and out a small aperture. Electrons scattered to a sufficient angle miss the hole and thus reduce the current measure. Careful current measurements therefore give a measure of gas density along the beams, and if an area is swept out by the pencil, then the flow characteristics over this area may be deduced. This method may, in fact, be considered a special case of the electron optical schlieren method, and is called bright field schlieren. It suffers from the intrinsic difficulty that one is attempting to measure a small increment of scattered current in the presence of the large unscattered current. Moreover, in the simple form it is a point-by-point method and hence the task of mapping a gas flow of any extent is rather tedious. However, it does lead us to the electron optical schlieren method, which is the principal concern of this report.

Historically, the first electron optical schlieren work was done by Boersch [29]. He used it to photograph carbon tetrachloride vapor jet at a pressure of about 1 Torr. Later, the method was used by L. Marton and his collaborators to observe small electric and magnetic fields of crystals [30, 31] and space charge in the cutoff magnetron [32]. Also, the first stroboscopic observations were made in connection with this latter work.

\section{The Principles of Electron Optical Schlieren}

All the methods of detecting gas flow by means of electron beams depend upon the fact that electrons are scattered by gas molecules. This scattering has been recognized as a minor annoyance in electronic devices from the earliest days of the electron optical art. The total cross section for scattering falls in the neighborhood of $10^{-15}$ $\mathrm{cm}^{2}$. It is worth noting that the existence of the total cross section implies that some of the electrons passing through a moderätely rarefied aggregation of gas molecules will not be perturbed in any way by the presence of the molecules. This result contrasts sharply with the results that occur when an electron beam passes through a cloud of charged particles. The charged particles produce a macroscopic field which affects every electron trajectory [33]. For low, uniform gas densities the total probability that an electron will be scattered is given by

$$
P_{s}=\sigma l N
$$

where $\sigma$ is the scattering cross section, $l$ is the electron path length, and $N$ is the number of molecules per unit volume. A rule-of-thumb measure of the total scattering probability is the fact that at 1 Torr and zero degrees Celcius the electron mean-free path is about $0.3 \mathrm{~mm}$ [34]. Thus, whenever the product of pressure in Torr and the electron path length through the gas is greater than $0.3 \mathrm{~mm}^{2}$, the electrons undergo on the average more than one collision passing through the gas. In this case, almost every electron carries some information in its altered trajectory. Devising the most efficient methods for gathering and interpreting this wealth of data constitutes a major challenge which we must decline in order to concentrate on the study of extremely rarefied gases.

If the effect of the pressure of the gas under study is lowered several orders of magnitude, that is, to $10^{-4} \mathrm{~mm}$ or below, the mean-free path of the electron through the gas is raised to the 
order of meter's. In most practical systems, then, the majority of the incident electrons must pass through the region of rarcfied gas without undergoing any scattering whatsoever. Study of the undeflected electrons cannot provide any information not already available from the study of the much less numerous scattered electrons. Even with idealized equipment in which the

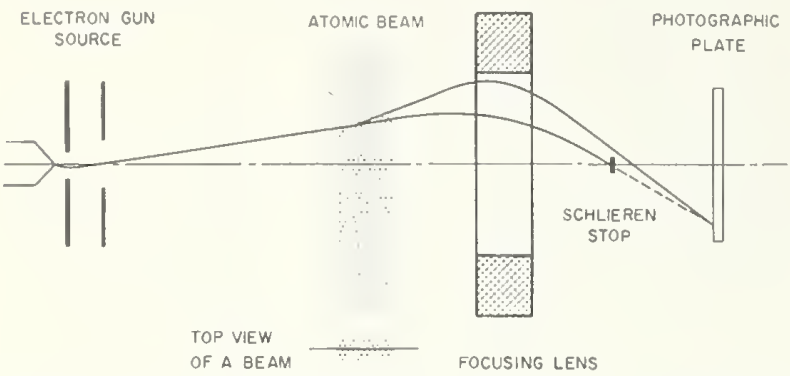

Figure 1. The basic electron optical schlieren technique employed in this experiment.

An electron bcam produced by the gun on the left is directed to cross the path of a molecular beam propagating in a dircetion perpendicular to the path of a molecular beam propagating in a dircetion perpendicular to the clectron bcam. In this interaction region, some ( 1 percent at $10^{-4}$ orr ) of the
electrons will be scattered through small angles by the gas molecules. The electron lcns is adjusted to focus the primary beam of electrons onto a small stop. Somc of the electrons whose paths have been altered will miss this stop and pass on to be registered on a photographic plate. The eleetron lens is arranged so that the mid-plane of the molecular beam is conjugate to that of the photographie plate thus producing an image in locus. The density at a given point on the image is proportional to the average gas particle density along the electron trajectory. electron beam intensity fluctuations and the variation of the detector efficiency are at a minimum, statistical mechanics requires that the presence of useless electrons represent noise to the detcctor and tends to mask the data [35]. It is clearly useful to have a discriminator which eliminates all electrons which have not been scattered at least once in the test region, while allowing the scattered electrons to pass without interference. The classic dark-field schlieren arrangement fills this requirement.

If a beam of electrons or of light consisting of precisely parallel rays or of rays diverging from a point source, as illustrated in figure 1 , is allowed to pass through an ideal converging lens, all of the rays must pass through a common point, the focus, after going through the lens. A solid stop of infinitesimal dimension located at this common point will capture or reflect all undisturbed rays passing through the lens. Any perturbation of a ray before it reaches the lens so that the position and slope of the ray that enters the lens does not coincide with the position and slope of any of the rays in the original bundle will cause the perturbed ray to miss the stop. The magnitude of the smallest perturbation which will cause a ray to miss the stop is proportional to the ratio of the stop diameter to the focal length. The minimum usable stop diameter in turn depends upon deviation of the beam from the idealized properties and upon the lens aberrations.

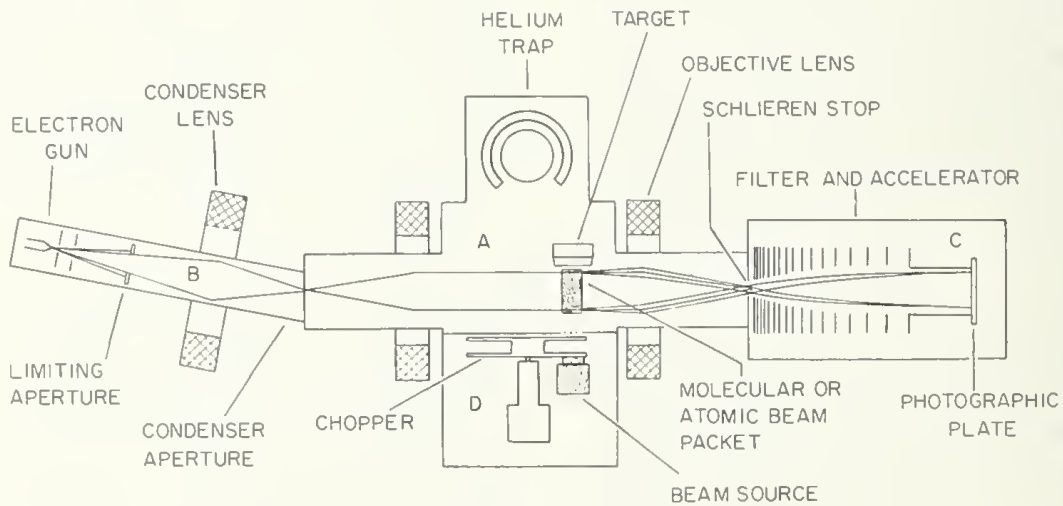

DARK FIELD SCHILIEREN TECHNIQUE

FIGURE 2. Overall schematic of the stroboscopic electron optical schlieren apparatus.

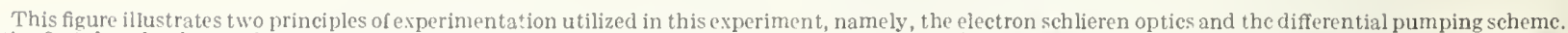

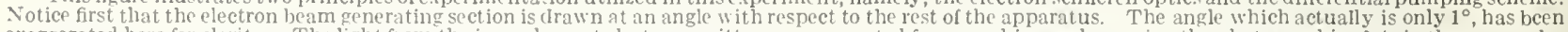

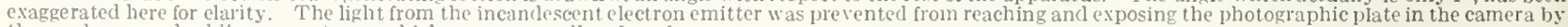
the condenser and schlieren apertures, which are very small and are far apart.

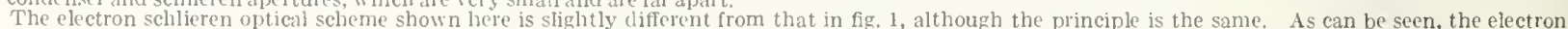

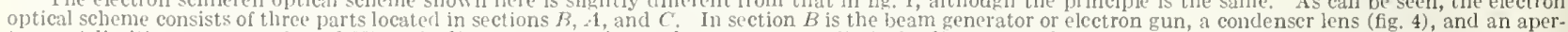

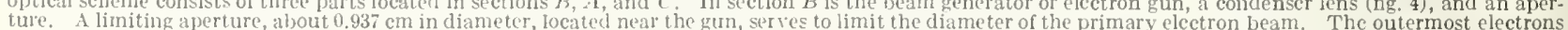
are thus prevented from striking the walls of the drift tubes

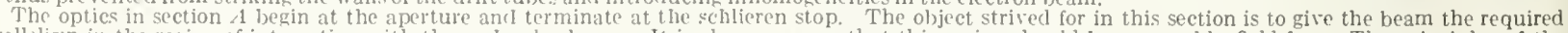

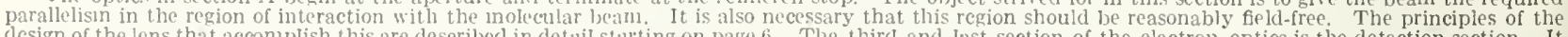

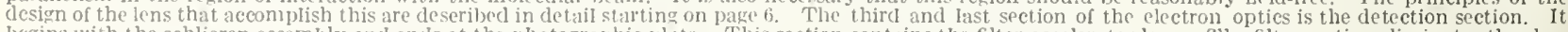

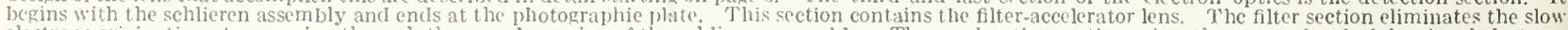

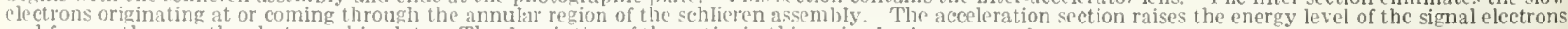
and focuses them on the photographic plate. The deseription of the opties in this region begins on page 6.

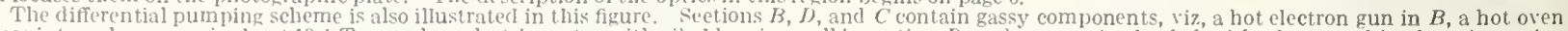

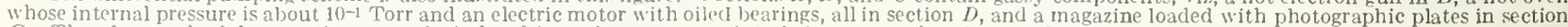

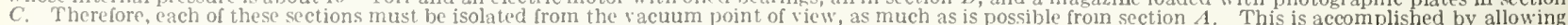

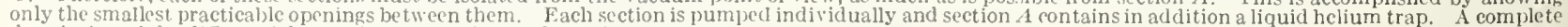
description of the differential pumping system is found starting on page 6 . 
From a theoretical light optical point of view [16], there is no great difficulty in designing lenses which can produce focal spots a few wavelengths in diameter. Problems of execution, such as producing or supporting a small stop, lens focusing and alinement, and mechanical perfection of the lens set the ultimate limit in the sensitivity of light optical schlieren. High sensitivity is needed in these instruments because the angular perturbation of the rays is quite small. The scattering of photons from individual molecules does not occur with usable probability; thus the perturbation of the light rays depends upon the gradient of the refractive index of the gas, considered as a continuous medium.

In the case of air, a pressure variation of 1 Torr in the distance of $1 \mathrm{~mm}$ normal to the light ray causes the light ray to bend with a $2.5-\mathrm{km}$ radius of curvature. 'Thus, the light optical schlieren observation is impractical because of the extreme precision of the required instrumentation in gaseous systems operating at pressures much below 1 Torr.

The situation is much more favorable in the case of electron optical schlieren. If an electron is scattered at all by the gas under study, it is usually scattered through a much larger angle than the available deflection in the light optical case. For example, in a rather unfavorable case where a $2 \frac{1}{2}-\mathrm{kev}$ electron beam is scattered by hydrogen atoms, simple classical theory, based on elastic scattering from a 'Thomas-Fermi atomic model, predicts that for about 50 percent of the scattered electrons the deflection angle exceeds 0.02 radian [36]. $\quad A$ discriminator which responds to this scattering angle will thus provide 50 percent of the maximum possible detection sensitivity, and there is no practical advantage in making this discriminator appreciably more sensitive.

At this point, perhaps it is well to emphasize the essential difference between the light optical schlieren method and the electron schlieren method. The light optical method depends on the gradient of the density whereas the electron optical schlieren is a measure directly of the density, since the scattering probablity depends on the density of the gas. Hence, although our electron optical schlieren photographs are produced by a true schlieren technique, their analysis is exactly the same as that of interferograms of light optics. When a parallel beam of electrons is brought to a focus onto the center of a stop by an ideal electron lens, those which were scattered through angles greater than the stop radius over the lens focal length will miss the stop, while the remaining electrons are captured. A reasonable size for this schlieren stop is something like $f / 100$ (see fig. 1).

In this simple theory we have considered the electron paths initially to be exactly parallel. This, of course, is an idealization; and it is a failure of this idealization which gives rise to some inconvenient variations of detection sensitivity. If the initial rays are not all parallel, the lens treats the rays as if they had already been scattered through
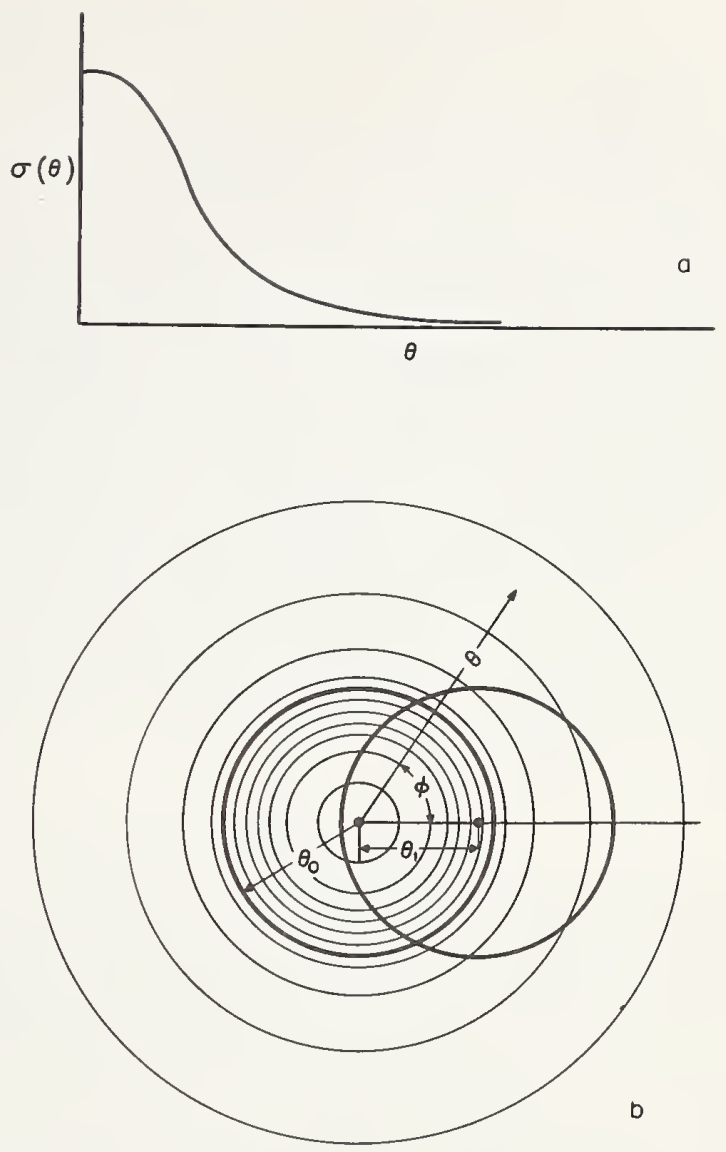

Figure 3.

(a) Schematic drawing of the differential scattering cross section $\sigma(\theta)$.

(b) Polar coordinate diagram of the information shown in fig. $3 a$ for the illustration of the role of aberrations in the optical system.

The information given in figure $3 \mathrm{a}$ is presented here in polar form. Scattering with a polar angle $\theta$ and the azimuth angle $\phi$ is represented by a vector, drawn from the origin with length $\theta$ and direction at the angle $\phi$ measured from the horizontal. The solid concentric circles represent lines of equal scattering cross section. The two dotted circles represent the region in scattering space resulting in eapture by the schlieren stop. Curve a represents the situation which exists if the unscattered ray hits the center of the seblieren stop. In this casc, electrons strike the stop or procecd to the photographic plate according as $\theta$ is less than or greater than $\theta_{0}$.

graphic plate according as $\theta$ is less than or greater than $\theta_{0}$.
Curve $b$ reprcsents the corresponding situation for rays which, although Curve $b$ represents the corresponding situation for rays which, although
undisturbed, strike the stop at the distance $\theta_{1} f$ from centcr. Here $f$ is the undisturbed, strike the stop at the distance $\theta_{1} f$ from centcr. Here $f$ is the
focal length of the focusing lens. The displacement is the result of non-ideal properties of the elcctron source and the focusing lenses. In this case, the critical value of $\theta$ for discrimination is a function of $\phi$. The electrons lost to the schlieren on the left side of the displaced eircle are more numcrous than those gained on the right side. Thus, the response of the photographic plate is slightly greater in case $b$ than case $a$. For small values of $\theta_{1}$, however, the difference nced not be great, since $\theta_{1}=0$ represents an extremum of the probability of eapture by the schlieren stop.

small angles, and hence the illuminating condition plays a role in determining the ultimate sensitivity. Moreover, if the lens lacks ideal focusing properties, as all electron lenses do, the parallel beams of electrons do not produce a point image. These deviations, known as lens aberrations, are more easily compensated for in light optics than in electron optics and indeed turn out to be one of the major linitations of the method. The roles of aberrations and imperfections in the optical system are illustrated in figure 3. 


\section{Realization of the Electron Optical Schlieren Method}

The efforts of the project divided naturally into two sections. 'The first, completed in about 1957 , was the realization of an instrument capable of visualizing a continuous molecular beam, at an equilibrium pressure of perhaps $10^{-5}$ Torr, existing in a background one order of magnitude lower. The second phase, which extended from this date until the end of the project, was that of demonstrating the feasibility of measuring the velocity distribution of a chopped molecular beam as it progressed across the field of view in the vacuum.

The problems in the two stages are essentially similar except that in the chopped-beam measurements one is working with an intensity some orders of magnitude lower, and the vacuum problem is complicated by the necessity of rotating mechanism, motors, etc., within the vacuum. The two will be discussed as a single problem until the problems special to the chopped beam are considered in a later section.

\subsection{Electron Optics}

The electron optics in both systems must perform three functions. First, an electron beam with reasonably well-defined properties is generated. The electron beam is propagated through the test region, where it is subject to the perturbation of the object space caused by the presence of the molecular beam. Finally, the properties of the beam emerging from the test region are measured. Each of these regions has its separate properties and will be dealt with in a separate section.

\section{a. Optics of the Beam-Generating Section}

The beam-generating section, shown schematically in figure 2, consists of an electron gun, a condensing lens, and a condenser aperture. The electron gun is required to give a uniform illumination which is without structure. It would be helpful if the electron gun produced only electrons and no other source of radiation. All electron guns operating with hot emitting elements, of course,

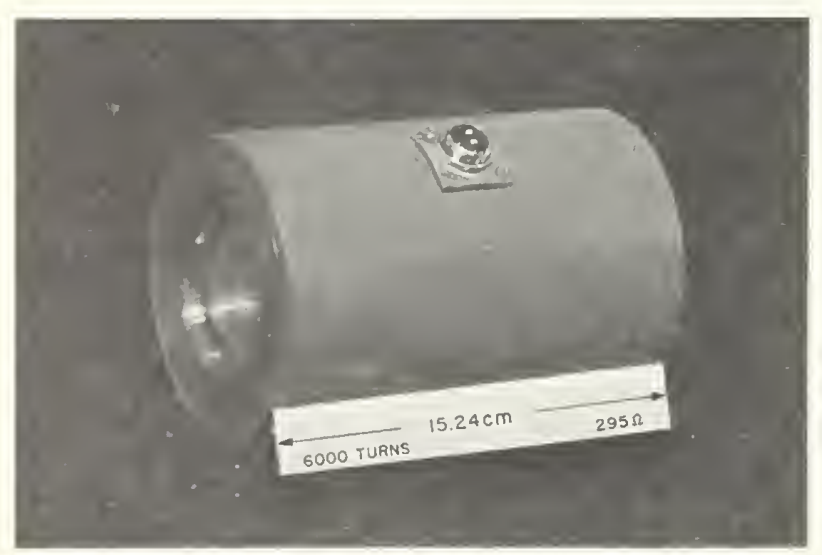

Figure 4. Iron-clad condenser lens. emit visible light. One of the problems which was met and overcome was that of preventing this light from reaching the photographic plate. For this purpose a bend was introduced into the electron optical column and apertures were used to eliminate straight paths through the system. In the first system, which used no small apertures except in the plane of the schlieren stop, a $4^{\circ}$ bend was required and corresponding deflection of the electron beam with a magnetic yoke was essential. Two small apertures were employed in the second instrument, which required only a $1^{\circ}$ bend. Although they were sometimes used, the magnetic yokes were not essential in this instrument. Operation without the correcting deflection merely shifted the exploring beam slightly sideways and provided no special problems. Since deflections tended to introduce beam structure, the deflection yokes were left de-energized for precise work. They were useful, however, for swift alinement of the instrument when precise results were not needed. A small deflection angle, $1^{\circ}$, and a small condenser aperture, $1 \mathrm{~mm}$, used simultaneously were sufficient.

The electron gun used for most of the work was a telefocus gun of the Siemens type [37]. This gun provides a well-defined crossover a short distance in front of the gun. This crossover was focused by the condenser lens into a small spot of higher convergence at the entrance to the test section. The existence of this small spot at the entrance to the test section made it possible to enhance the vacuum in the test section by means of differential pumping. The electron beam entered the test section through an aperture only $1 \mathrm{~mm}$ in diameter. The lens which was adopted for this purpose was a conventional iron-clad magnetic lens designed roughly according to the criteria of Liebmann [38] (see fig. 4).

Throughout the course of the investigation we were plagued by minor inhomogeneities in the electron beam. Many of these were caused by small deviations from cylindrical symmetry in the vicinity of the gun cathode. Small irregularities at the condenser crossover and at the schlieren stop also contributed to these disturbances. In most cases the beam inhomogeneities are of too slight contrast to be observed on a fluorescent screen. Thus it is not surprising that they have been ignored in the literature of electron optics.

\section{b. Optics of the Test Section}

The small electron spot is placed at the focal point of the first of two principal lenses which straddle the interaction space. It is the purpose of the first lens to give the required parallelism to the beam at the point of interaction with the atomic beam. The second coil focuses the beam on the schlieren stop. It is also required that the atomic beam be in a reasonably field-free region. Hence, these lenses are double-coil lenses so arranged that the field has a stationary value of 
zero at the mid-plane of the molecular beam. The design problem in these lenses, which have reasonably long focal lengths is to place the center of this region of nearly zero field at an acceptable position for the object plane of the second lens. The following paragraph gives the design of these lenses in some detail.

The coil system was designed around the requirement that the magnetic field strength should be as near as possible to zero throughout the region occupied by the molecules under study in order to provide straight electron trajectories through the test region. With two coils of equal diameter, acting in opposite directions, we were able to produce a lens field with a peak axial field strength more than 300 times greater than the strongest axial magnetic field in the region occupied by the molecular beam. The method used was to derive a set of required relationships between the various coil parameters such that at the mid-plane of the atomic beam, i.e., at the object plane, the axial magnetic field $H(z)$ and its first derivative $H^{\prime}(z)$ should be zero.

The axial magnetic field produced by a coil of $n$ turns of radius a carrying a current $i$ expressed in magnetic units is

$$
H(z)=\frac{2 \pi n i a^{2}}{\left(a^{2}+z^{2}\right)^{3 / 2}}=\frac{H_{0}}{\left(1+\frac{z^{2}}{a^{2}}\right)^{3 / 2}} .
$$

For less cumbersome notation, let us write $z$ in units of the coil radius $a$. Then

$$
\begin{gathered}
H(z)=\frac{H_{0}}{\left(1+z^{2}\right)^{3 / 2}} \\
H^{\prime}(z)=\frac{d H}{d z}=\frac{-3 z H_{0}}{\left(1+z^{2}\right)^{5 / 2}},
\end{gathered}
$$

and

$$
\frac{H^{\prime}(z)}{H(z)}=\frac{-3}{z+1 / z} \text {. }
$$

Let the distance from the object plane to the respective coil planes be $z_{1} a$ and $z_{2} a$, respectively. For the field and its first derivative to vanish simultaneously, we must have $H\left(z_{2}\right)=-H\left(z_{1}\right)$ and $H^{\prime}\left(z_{2}\right)=-H^{\prime}\left(z_{1}\right)$. Hence

$$
\frac{H^{\prime}\left(z_{2}\right)}{H\left(z_{2}\right)}=\frac{H^{\prime}\left(z_{1}\right)}{H\left(z_{1}\right)}
$$

Using the formula for $H^{\prime} / H$, we find that this result yields $z_{1}+1 / z_{1}=z_{2}+1 / z_{2}$. Solutions are

$$
z_{1}=z_{2} \text { and } z_{1}=1 / z_{2} \text {. }
$$

The first solution is trivial, as the coils then coincide and produce equal and opposite fields everywhere. The second solution gives a family of possible useful solutions. The condition $H\left(z_{2}\right)=-H\left(z_{1}\right)$ is met by running currents through the coils in opposite senses and correctly choosing the turns ratio $R=n_{2} / n_{1}$. Thus

$$
\frac{1}{\left[1+z_{1}^{2}\right]^{3 / 2}}=\frac{R}{\left[1+z_{2}^{2}\right]^{3 / 2}}=\frac{R}{\left[1+\frac{1}{z_{1}^{2}}\right]^{3 / 2}}=\frac{z_{1}^{3} R}{\left[1+z_{1}^{2}\right]^{3 / 2}} .
$$

Without further consideration, the choice $z_{1}=$ $1.25, z_{2}=0.8, R=0.512$ was made as the normalized distance between coils $\left(z_{1}-z_{2}=0.45\right)$ and the normalized distance from the object plane to the nearest lens $\left(z_{2}=0.8\right)$ were convenient. A 10-in. mean coil diameter was then selected as the largest compatible with the design of the main chamber. (The coils had to be able to slide up to the outer wall of the chamber without being stopped by the top flange of that chamber.)

The stray axial field at a distance $z$ from the object plane is given by

With

$$
H_{\mathrm{str}} \cong\left[H^{\prime \prime}\left(z_{1}\right)+H^{\prime \prime}\left(z_{2}\right)\right] \cdot \frac{z^{2}}{2 a^{2}} .
$$

this becomes

$$
H\left(z_{1}\right)=H_{0} K\left(z_{1}\right)
$$

$$
H_{\mathrm{str}}=H_{\mathrm{o}}\left[K^{\prime \prime}\left(z_{1}\right)-R K^{\prime \prime}\left(z_{2}\right)\right] \frac{z^{2}}{2 a^{2}} .
$$

By simple differentiation,

$$
K^{\prime \prime}(z)=\frac{3\left(4 z^{2}-1\right)}{\left(1+z^{2}\right)^{7 / 2}}
$$

With the adopted values of $z_{1}, z_{2}$, and $R$, we then have $H_{\mathrm{Btr}}=0.0805 H_{\mathrm{o}} \frac{z^{2}}{a^{2}}$. With $a=10 \mathrm{in}$. and $z=0.5$ in. at the extremity of the molecular beam, $H_{\text {str }}$ reduces to $0.00201 H_{0}$, while the peak field occurs at $z_{1} \cong-0.15$ and has the value 0.643 $H_{0}$.

Rough computations for the spherical aberration constant of this coil system, used as a lens, gave $S_{\mathrm{Bph}}=0.0107 / \mathrm{cm}^{2}$. With a beam radius of 1.25 $\mathrm{cm}$, this leads to a circle of confusion [39]

$$
\Delta r_{f}=\frac{1}{4} S_{\mathrm{sph}} r^{3}=0.0052 \mathrm{~cm}
$$

In the symmetrical arrangement of two sets of lenses, as used in this experiment, the $\Delta r$ 's of the two lenses add, and the circle of confusion due to the spherical aberration of the condenser lens, as seen at the condenser aperture, is imaged at unity magnification. The Seidel coefficient for this lens was computed to be $S_{\mathrm{sph}}=0.0184$. The beam radius in passing through this lens is $0.625 \mathrm{~cm}$, and the radius of the circle of confusion produced by the condenser lens is thus

$$
\Delta r_{c}=0.0011 \mathrm{~cm} \text {. }
$$

The circle of confusion on the schlieren stop is then

$$
\Delta r_{s}=2 \Delta r_{f}+\Delta r_{c}=0.0115 \mathrm{~cm}
$$


Our practical experience in manipulating the focus in the vicinity of the condenser aperture indicated that the crossover diameter in that plane was about $0.05 \mathrm{~cm}$. We believe that the discrepancy between this result and $\Delta r_{c}$ above is due to the finite source size in the electron gun and spherical aberration in the gun, although no figures on these aspects of gun performance were available.

\section{c. Optics of the Detection Section}

Figure 5 is a photograph of the schlieren stop used. In this case, a scroll cup about $2 \mathrm{~mm}$ in diameter was made from a platinum sheet and spot-welded onto a heavy platinum wire. In earlier work the stop was made from sheet molybdenum spot-welded onto a tungsten rod. The platinum cup had the advantage that it shielded the electron beam from the ever-present brown deposit that forms whenever an electron beam spot hits a surface in a dynamically pumped system. This deposit is capable of storing a charge and therefore of affecting the electron image on the plate. Further, the platinum stop can very easily be cleaned by simply heating to a red glow

It can be shown that a high photographic efficiency and a high scattering cross section are mutually contradictory requirements. Scattering cross section falls rapidly and photographic efficiency increases rapidly as the electron energy is increased. The resulting overall response of a simple electron schlieren camera is rather poor at all beam energies. Under typical conditions a 30 -min exposure is required for a barely satisfactory photograph of a molecular beam with
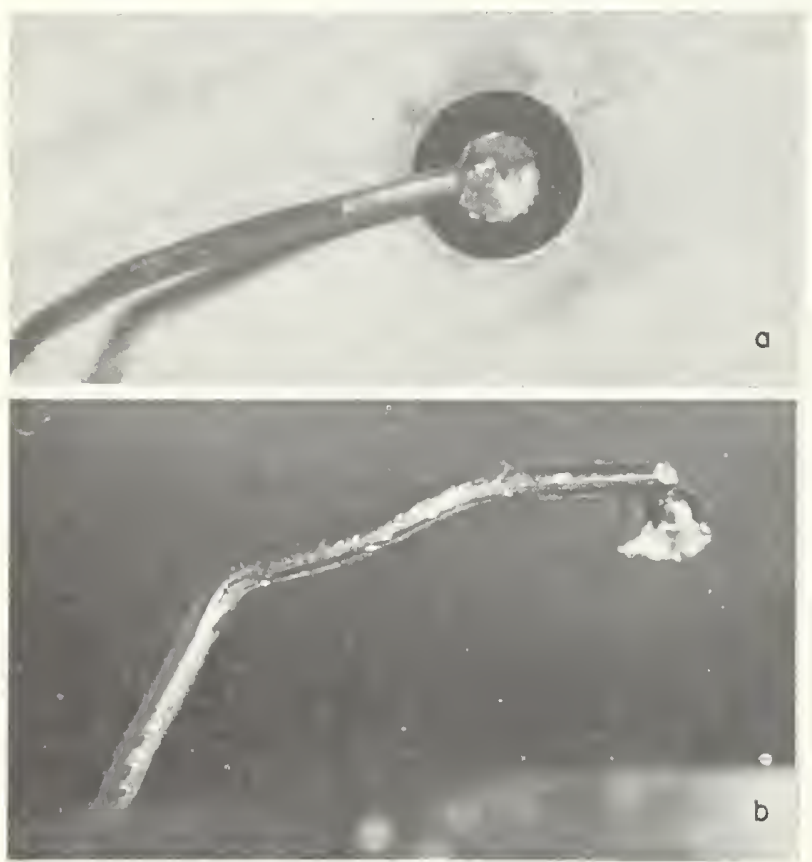

Figure 5. Two views of the schlieren stop and aperture.

Dimensions of parts are cup diameter, $1 \mathrm{~mm}$; aperture diameter, $3.9 \mathrm{~mm}$; and sheet thickness $0.38 \mathrm{~mm}$. density corresponding to $10^{-4}$ 'Torr pressure. To secure the advantages of both high and low bean energies, an electron accelerator lens is inserted immediately following the schlieren stop. The beam energy in the scattering region may thus be kept conveniently low for high scattering cross section, while energies of $10 \mathrm{kev}$ or more are employed to expose the photographic plate.

If the beam energy in the scattering region is made extremely low, however, the experimentalist is plagued by a variety of annoyances. Stray electrostatic and magnetic fields deflect the beam to an increasing extent, and space charge limits the available beam current. The magnetic field of the earth is opposed by an equal field generated by compensating coils (see secs. 13.2 and 13.3), but additional magnetic fields are introduced by ferrous materials used in the vacuum pumps and electronic equipment. Since these materials have high magnetic permeability, their internal fields are negligible in comparison with the external field. Consequently, the scalar magnetic potential on the surface of a ferromagnetic object is essentially constant. In the case of a sphere of volume $\dot{V}$, in the presence of the earth's field $H_{e}$, this condition is equivalent to a dipole of strength $M=V H_{e} / 8 \pi$ at the center of the sphere. Thus at a distance $\ell$ from the center of the sphere, the disturbing field varies from $V H_{e} / 8 \pi \ell^{3}$ to $V H_{e} / 4 \pi \ell^{3}$, depending upon direction of the displacement. For objects of different shapes, this formula for the stray field is inexact, but it does give the correct order of magnitude. On this basis we estimate that non-uniform fields of the order of 10 mgauss were present in the scattering area of our instrument.

Electrostatic fields may be produced by charging of nonconducting impurities on metal surfaces or by differences of surface potential on different crystal planes of the metal. Such effects are not too well understood and certainly not easy to control. Thus, while meticulous attention to stray electrostatic and magnetic fields would probably provide satisfactory optical conditions at considerably lower voltages than we used, it was more practical in this experiment to raise the operating voltage to a level at which these disturbances could be tolerated. By trial and error, a beam energy of about $2 \mathrm{kev}$ in the scattering area was found to work best in our instrument. Somewhat less satisfactory operation was possible at voltages ranging downward to $1000 \mathrm{v}$. At voltages below this minimum operating point, image distortions and alinement difficulties were extreme. The acceleration of the beam from 2 $\mathrm{kev}$ in the scattering region to $12 \mathrm{kev}$ at the photographic plate reduces the required exposure time from 30 min to a few seconds. The improvement, which indeed is vital to the success of the method, is not without its inherent difficulties. An accelerating field in the region of the schlieren stop picks up electrons which arise from the intense beam striking the schlieren stop, accelerates them, and forms an intense inhomogeneous background on the photographic plate. To avoid the back- 
ground effect, we designed the lens to have a decelerating or a filtering stage before the accelerating stage. Since most of the electrons arising at the stop are either secondaries or electrons which have transferred energy to the stop, they are of significantly lower energy than the primary electrons and may be removed by modest energy filtration. Because of the importance of this stage, its design is given in detail.

The mechanical features of the post-acceleration lens are shown in figure 6. A series of 17 copper apertures supported on three glass insulating rods provides controlled potentials on the boundary of the region in which the acceleration takes place. The first aperture plate sits directly in front of the schlieren stop and is at ground potential, while the last plate is attached to a tube extending into the drift space leading to the image plane. It is at the potential of the camera. A short glass tube insulates the camera assembly from the main instrument housing, which is at ground potential. By applying various potentials on the aperture plates, a wide variety of axial potential distributions may be produced. Electrode potentials which are positive with respect to ground are produced by a resistance bleeder connected to a $30-\mathrm{kv}$ positive supply and fed to the plates through kovar seals. Six negative potentials are derived from a bleeder consisting of wirewound, 1-megohm rheostats connected to a negative $150-\mathrm{v}$ regulated supply. The outputs of both supplies were adjustable from zero to the maximum voltage. The resistors in each leg of the bleeder chain were pairs of 2 -w carbon resistors. One resistor of each pair was chosen to be just short of the desired resistance and the other, much smaller resistor was selected to bring the combination to the desired resistance. The resistance values were checked on a precision Wheatstone bridge. Thus we constructed an inexpensive voltage divider of better than 1 percent initial precision from resistors of 10 percent manufacturing tolerance.

We chose the electrode potentials in a manner designed to simulate an axial potential distribution with desired focusing properties. Since the electron beam is most easily disturbed in the lowvoltage portion, we devoted more apertures to control of the low-voltage portion of the field than to the high-voltage portion. Thus, instead of being spaced uniformly, the plates were spaced at exponentially increasing distances from one another. The hole diameter increased uniformly from plate to plate.

The field was required to accelerate the electrons which missed the stop to the desired final potential and to reject any secondary electrons produced at the stop. In addition, it was required to provide a focused image of the object plane with minimal distortion at the fixed image plane. With the schlieren lens spacing and focal length, as employed in the instrument, the lens had to work with a virtual object approximately $38 \mathrm{~cm}$ before the first plate. The image plane was $9.05 \mathrm{~cm}$ beyond the camera flange entrance.

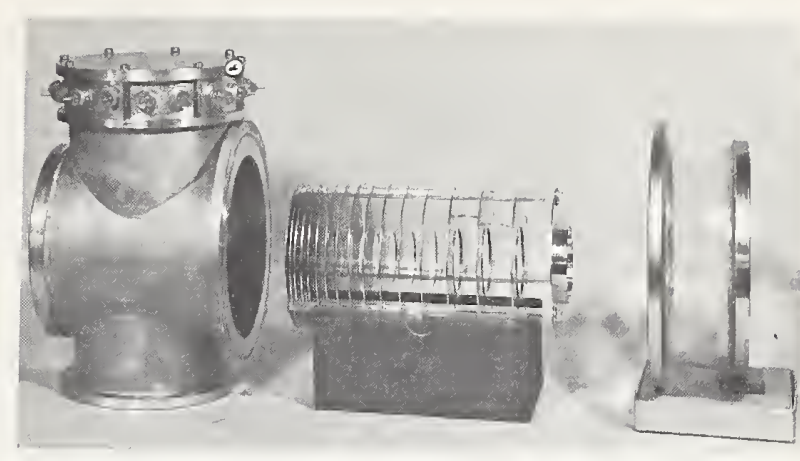

Figure 6. Exploded view of the filter accelerator.

At left is the housing which has provision for 20 lead-in connections. Next is the disk assembly. On the right is the glass insulator section. The glass cylinder is made of Pyrex and sealed into the brass rings with an epoxy resin. The camera bolts onto the flange on the right.

Experience with the first instrument had shown us clearly that if the lens fields are strong enough to form an intermediate image in addition to the final image, intolerable pincushion distortion is produced. If only one image is formed, the pincushion distortion is of suitable size to be balanced out by the barrel distortion produced by the schlieren lens coil. In operating the lens without any intermediate images, the ratio of the final electron energy to the initial energy is limited by the need to keep the lens weak. Since photographic efficiency increases rapidly with voltage ratio, it is clear that we must seek the maximum voltage ratio consistent with the required object and image positions, thus permitting us to attain focus at the highest possible accelerating voltage. From the point of view of simple lens theory, the axial potential distribution $\phi=\phi_{0} e^{k z}$ appears superficially to be very attractive. The modified Gaussian ray solutions $u=r\left(\phi / \phi_{0}\right)^{1 / 4}$ are the sine and cosine of $\frac{\sqrt{3}}{4} k z$. With these facts, it is easy to show that if the exponential solution is fitted to the initial and final potentials at the extremities of the lens, focusing occurs at a voltage ratio of 20.4. The field is, however, quite unusable, since no provision is made for the rejection of secondary electrons; and the sudden termination of the field at the plane of the camera flange would require a small aperture at that position. To reject secondary electrons we lower the axial potential slightly in the region just following the stop. Lowering the potential by only a few percent is sufficient to stop all of the secondary electrons, since they leave the stop without forward momentum. It is desirable, however, that the field should vanish in the vicinity of the schlieren stop. The simplest field form with this property is

$$
\phi(z)=\phi_{0}-a z^{2}+b z^{3} .
$$

The general shape of this field in the neighborhood of the schlieren stop is shown in figure 7 . For 


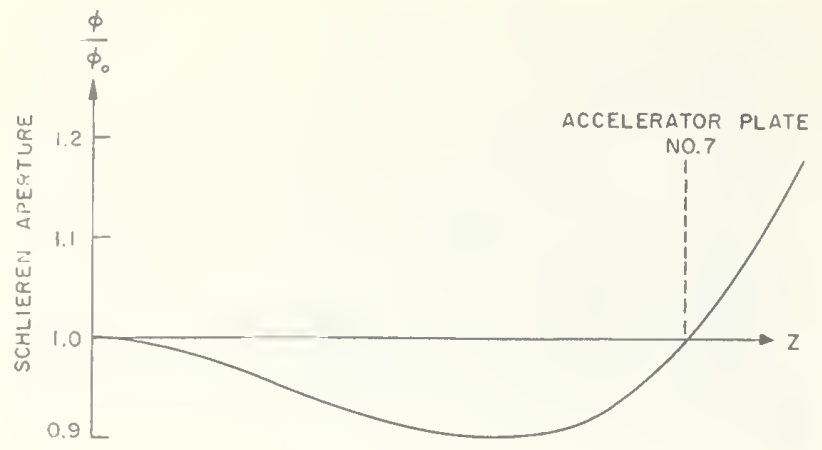

Figure 7. Decelerating and transition field, as given by $e q(4.12)$.

adequate control of the contours of this field as far as the point where $\phi=\phi_{0}$ with $\phi^{\prime}(z)>0$, we assigned the first six electrodes to this section of the field. For the remainder of the field, we wanted $\phi^{\prime} / \phi$ to build up to a maximum and then fall asymptotically back to zero. The asymptotic return of $\phi^{\prime} / \phi$ to zero should match the roughly exponential falloff of field in the drift tube beyond the camera flange.

A field for which the Gaussian trajectories are known analytically and which essentially satisfies these requirements is given by

$$
\phi_{0} \exp \left[\frac{4 k_{0} a}{\sqrt{3}} \tan ^{-1}\left(\frac{z}{a}+\frac{\pi}{2}\right)\right]
$$

which provides an acceleration voltage ratio

$$
\phi_{2} / \phi_{1}=\exp \left[\frac{4 k_{0} a}{\sqrt{3}}\right] .
$$

This field has the property that

$$
\frac{3}{16}\left(\frac{\phi^{\prime}}{\phi}\right)^{2}=-\frac{u^{\prime \prime}}{u}=\frac{k_{0}^{2}}{\left[1+\frac{z^{2}}{a^{2}}\right]^{2}},
$$

where $u=r^{1 / 4}$, and $r(z)$ is any paraxial ray solution.

The solution for the magnetic lens with $-r^{\prime \prime} / r$ given as the same function of $z$ has been found analytically by Glaser [40]. Experimenting by trial and error with various values of $k_{0}, a$, and the position of the origin in the lens field, we were able to choose the most suitable potential distribution from this family of potential curves. To be acceptable, a potential curve had to provide an image of the object in the image plane, had to match the potential and field of an acceptable solution (4.11) at the seventh electrode, and had to approach uniform potential in a realistic manner in the drift tube leading to the image plane. The axial potential in a region surrounded by a conducting tube is approximated by [41]

$$
\phi=\phi_{0} \exp -\left[\frac{n_{1} z}{r}\right]
$$

where $r$ is the radius of the tube and $n_{1}$ is the first root of the zero-order Bessel function $J_{0}(x)$.

The field given by our family of potential distributions falls off too slowly at large values of $z$ to match the exponential decline of a field inside a conducting cylinder. To make our model realistic, we modified the field to the form

$$
\phi=\phi_{0} e\left[\mathrm{k} \int_{-\infty}^{z} \exp \left(-\frac{x^{2}}{\lambda^{2}}\right) d x\right]
$$

in which

$$
\frac{3}{16}\left(\frac{\phi^{\prime}}{\phi}\right)^{2}=\frac{3}{16} k^{2} \exp \left(-\frac{2 z^{2}}{\lambda^{2}}\right)
$$

The focusing power thus has a bell-shaped distribution similar to $(4.12,4.13)$, but fades out much more rapidly at high values of $z$. 'This field thus readily fits the boundary condition in the drift tube. If this field is fitted to match (4.12) in the neighborhood of $z=0$, we have a realistic field which can be matched at the lowvoltage end by a suitable polynomial function which provides the retarding potential. The focusing characteristics of this "error-function" field differ only slightly from the characteristics of the matching accelerating field. A rough check on the change in focusing characteristics is obtained by computing trajectories for a field of form (4.12) which is terminated at the points where $\phi^{\prime 2} / \phi^{2}$ in the error-function field falls to half of the corresponding value in (4.13). The focusing effects of the retarding field given by the polynomial function prove to be so slight that they may be neglected in the design calculations.

Having developed a piecewise-analytic expression for the axial potential distribution, we are ready to compute the potentials to be applied to the plates. For this purpose, we utilize the series expansion

$$
\phi(r, z)=\phi(0, z)+\frac{r^{2}}{2^{2}} \phi^{\prime \prime}(z)+\frac{r^{4}}{2^{2} \cdot 4^{2}} \phi^{I V}(z)+\ldots
$$

which gives the radial variation of $\phi$ as required by the Laplace equation. The required electrode potentials are thus found to differ slightly from the potential desired at the axial position at the same value of $z$. The corrections are, however, so small that only the first two terms of the series are needed. Because of the small degree of correction needed, we are justified in assuming that the actual $\phi(z)$ on the axis deviates from the planned $\phi(z)$ by only a few percent. The properties of the designed field, together with the electrode dimensions, positions, and potentials are given in the following table: 


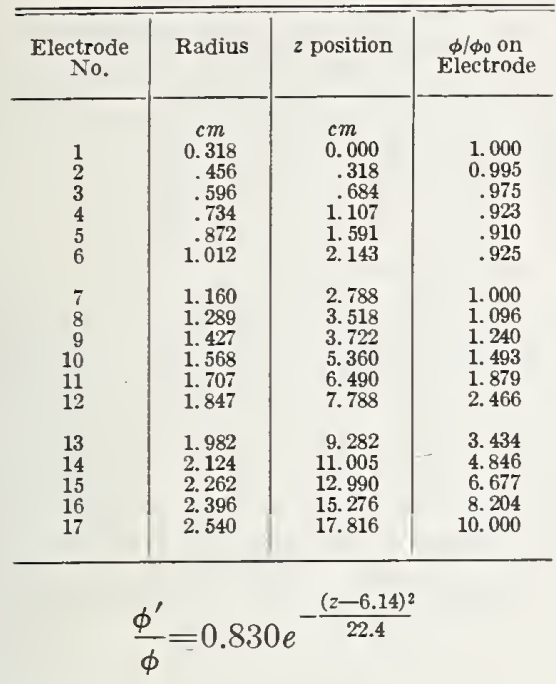

Axial potential:

$$
\begin{gathered}
\frac{\phi}{\phi_{0}}=0.966 \exp \left[\int_{2.79}^{z} e^{-\frac{(x-6.14)^{2}}{22.4} d x}\right] \\
\frac{\phi}{\phi_{0}}=1-0.076 z^{2}+0.0258 z^{3} \quad(z \geqq 2.79)
\end{gathered}
$$

In actual operation the post acceleration potential is adjusted as needed to secure exact focus. The actual potential distribution then no longer belongs to the family of distributions which formed the basis of our calculations. The design can be considered satisfactory, however, as long as the actual voltage ratio for the in-focus condition is a major fraction of the calculated ratio. This condition was fulfilled.

Terminating the accelerator stage is the camera housing. The requirement that the interaction space be at ground potential necessitates that the final stage of the accelerator and the camera be at $12 \mathrm{kv}$ above ground. The insulation is achieved by inserting a glass section in the vacuum wall. The camera housing is provided with a protective shield to prevent accidental contact by the operator, and a safety interlock to shut off the high voltage when the camera is to be operated. The camera serves two functions: it contains and manipulates the photographic plates for recording the data, and it provides a fluorescent screen on which the operator can view the image. The photographic material used in this device was process plates, $6.5 \times 9 \mathrm{~cm}$. Construction details and photos of this camera are given in section 13.2 .

\section{Gas Kinetics}

The electron optical schlieren system, as described in the preceding sections, is a sensitive device for mapping gas density distributions at extremely low pressures. Exposures of 2 or 3 secs are sufficient to provide normal exposures in the presence of gas densities lower than $10^{-6}$ Torr. The chief barrier to operation at pressures four orders of magnitude lower is simply the need to keep the background vacuum at that level. Furthermore, the schlieren camera can be used with a shutter speed of a microsecond or less if required.

To test the spatial resolution capabilities of this instrument, one needs a sharply defined static formation of gas molecules. The conventional molecular beam is ideally suited for this purpose. Molecules escaping into a vacuum from a small opening in the side of a chamber containing gas at a moderately low pressure travel in straight lines from the opening, provided that the pressure inside the source chamber is sufficiently low. The majority of the atoms will leave a slit of width $w$ and travel in a straight path until they hit a solid surface, provided that the mean free path $\lambda$, corresponding to the ambient pressure within the source chamber, is large in comparison with the slit width $\lambda \cong 10 w$ [42]. A second slit parallel to the source slit captures all molecules whose paths do not lie within a ribbon-shaped region limited by the two sets of slits. If precautions are taken so that molecules completing their trip are not allowed to return to the interaction space, the resulting molecular beam is sharply defined in space with an easily calculated density distribution. The sharp density gradient at the edges of the beam provides a critical test of the resolving power of any detection system for rarefied gases.

For testing the time-resolving ability of the schlieren system, we need to vary the gas formation rapidly with time in a reproducible manner. If the beam is divided into segments by means of a rapidly rotating wheel with radial slots in the beam path, the segments will move in the direction of beam propagation. The modified Maxwellian velocity distribution of the individual molecules will, however, cause the molecular beam segments to spread almost as rapidly as they propagate. A second slotted disk rotated at the same speed as the first, but with the slots phased to pass through the beam at an appropriate later time, will limit the beam pulses to those molecules whose velocity was just right to pass from one disk plane to the other in the chosen delay time. By control of this delay time and the width of the chopper slits, any mean velocity present in the original beam and any velocity spread within reason may be selected. The restriction of the velocity spread to a narrow range entails a corresponding loss of beam intensity. The geometric spreading of a beam as it leaves the source slit causes the density without chopping to fall to a value somewhat lower than 
$\ell / w$ times the density at the source slit, where $\ell$ is the distance from the source slit to the point in question. It is thus evident that when the density is further reduced by approximately $\delta v / v$, where $v$ and $\delta v$ are the mean value and spread of the pulse velocity, the maximum possible density of atoms in the pulse corresponds to a mean free path

$$
\lambda=10 \ell \cdot v / \delta v \text {. }
$$

Since cross sections for electron scattering are an order of magnitude lower than those for molecular collisions, the proportion of electrons scattered in passing through a chopped beam is only

$$
\sigma=\frac{\Delta z}{\lambda}=\frac{\Delta z \delta v}{100 \ell v}
$$

under ideal conditions. Inserting typical experimental conditions, viz, $v=3 \cdot 10^{4} \mathrm{~cm} / \mathrm{sec}, \delta v=1 \cdot 10^{4}$ $\mathrm{cm} / \mathrm{sec}, \Delta z=2.5 \mathrm{~cm}, \ell=4 \mathrm{~cm}$, yields $\sigma=1 / 480$, and it is thus evident that every effort must be made to maintain high intensity in the chopped beam.

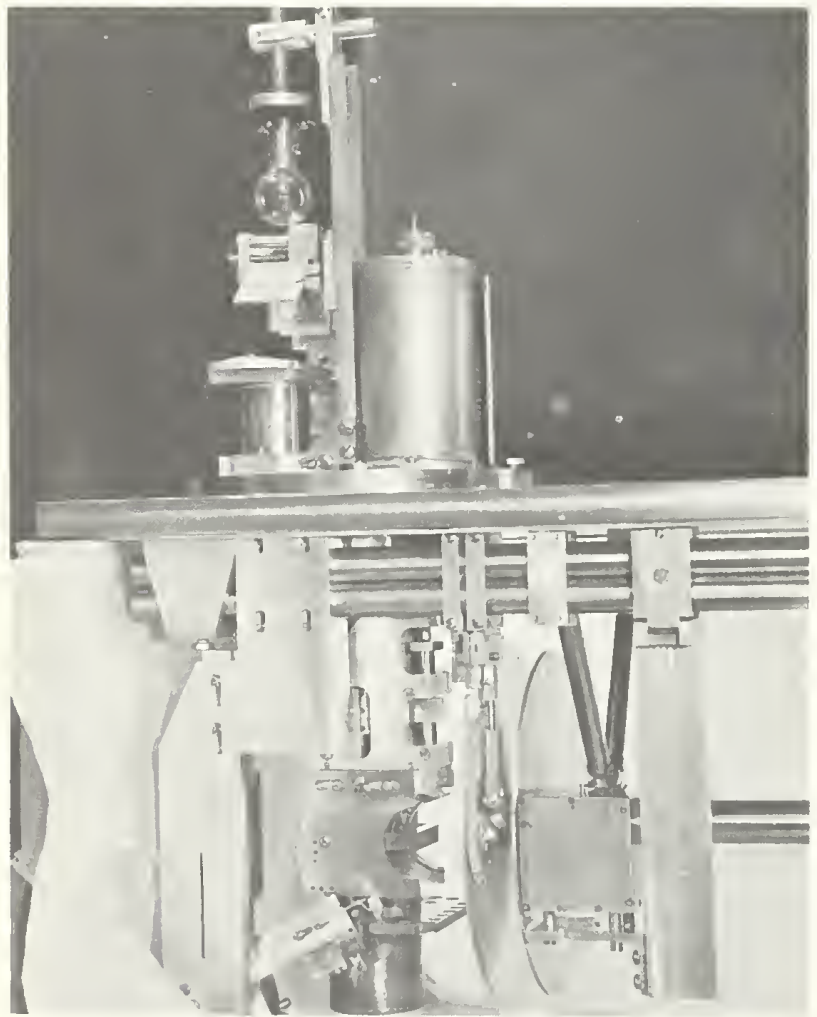

Figure 8. Photograph of components suspended from the top of the main chamber.

Starting from leit to right one can scc first the mirror asscmbly of the Cassegrainian light optical triggcring systcm. Next is the target assembly, and behind that a liquid nitrogen trap can be seen on the botton. The septum has been removed from this picture to reveal the set of chopping distis, and the components located between the disks, namely the molecular beam collimator slits and mirror $\boldsymbol{M}_{t}$ of the Cassegrainian optics system. Behind the first chopping disk the oven with its shield and supports can be seen. Abovc the top plate are the control rods mounted on stainless steel bcllows and to the left of those are the various structures of the light optical system. The mirror $M_{4}$ is identified in figure 10 .
If the beam is finally used to study accommodation and viscous slip coefficients by reflection from solid targets, the relatively compact beam before impact is spread out over most of the field of view and another factor of 20 to 200 in beam intensity is lost.

\subsection{Beam Source}

The cadmium molecular beam had the shape of a ribbon running horizontally across the electron path. Its cross-sectional measurements were $1.3 \mathrm{~mm} \times 35 \mathrm{~mm}$, with the long dimension ir the direction of electron flow. A metal septum fitted with slits very slightly wider than the molecular beam thickness separated the highvacuum interaction space from the relatively gassy molecular-beam-generating components, which were separately pumped. 'The molecular beam was generated in a conventional oven with two sets of collimating slits. The oven was machined from OFHC copper into a hollow rectangular cube having a removable top to allow loading of the oven. A slit opening $3.3 \mathrm{~mm} \times 25.4$ $\mathrm{mm}$ was machined and a set of adjustable collimating slits was mounted over it. The slit opening was set to be $0.5 \mathrm{~mm} \times 25 \mathrm{~mm}$. The oven was heated by a set of tungsten coils encased in alumina tubes to provide insulation. The heaters were inserted into holes drilled into the oven located along the outer edges of four sides and parallel to the slit. A re-entrant tube into the oven chamber was provided to contain a thermocouple used for temperature control and measurement. 'The oven was heated by unfiltered rectified a-c current controlled by a commercial automatic temperature regulator.

The oven was supported kinematically on top of three rounded stainless steel screws which bore into $\mathrm{V}$ slits milled across the bottom of the oven. A spring pressure contact on top of the oven insured that the oven would bear firmly against its supports. Adjustments of oven position and tilt were made by turning the supporting screws. Since contact of the oven with its surroundings was limited to seven point contacts with stainless steel, which is a poor conductor, conductive heat loss was low. To reduce the heat loss further, a stainless steel shield was built around the oven, and the base for the mounting screws and stainless steel shield was mounted on thin stainless steel tubes, which are good insulators (see fig. 8).

The dimensions pertinent to the formation of the molecular beam and a beam packet are as follows: oven slits, $1 \mathrm{~mm} \times 25 \mathrm{~mm}$; first chopper blade slits, $2 \mathrm{~mm} \times 22 \mathrm{~mm}$ and about $5.1 \mathrm{~mm}$ from oven slits; collimating slits, $0.5 \mathrm{~mm} \times 25 \mathrm{~mm}$ about $20 \mathrm{~mm}$ from first chopper blade; second chopper blade slits, $0.5 \mathrm{~mm} \times 25 \mathrm{~mm}$ set as close as possible to, but not touching, the collimator (estimated $0.5 \mathrm{~mm}$ ); and finally, the septum slits, $0.5 \mathrm{~mm} \times 25 \mathrm{~mm}$ about $2.5 \mathrm{~mm}$ from second chopper blade. In the continuous-beam work, the dimensions are the same but chopper disks are removed. 
For the bulk of the experimental work the material used in this oven was cadmium. The condensation properties of this material were both an advantage in lowering the residual background pressure and a handicap, in that the more sophisticated ovens [43] using parallel channel flow as developed by numerous workers were unsuitable for us because of condensation of the cadmium vapor in the long narrow channels. With our oven, we obtained a molecular beam which was of sufficient density to be useful in the steady state and chopped conditions.

In addition to the work with the cadmium vapor, preliminary experiments were run using carbon dioxide and air from a similar source. Once again, the technique was successful in detecting the presence of these continuous molecular beams.

\subsection{Chopped Beams}

At the temperatures at which the beam-source oven is operated, the mean velocity of the molecular beam is approximately $300 \mathrm{~m} / \mathrm{sec}$, with a velocity spread characteristic of the oven temperature of approximately $320^{\circ} \mathrm{C}$. In order to do significant time-resolved work, it is necessary not only to pulse the beam, but also to monochromatize the beam to such a degree that a limited range of velocities may be assigned to the members of the packet. The velocity selection is done in the classic manner by using two offset slits on the periphery of a pair of rotating disks. In order that a reasonable ratio of geometrical pulse length

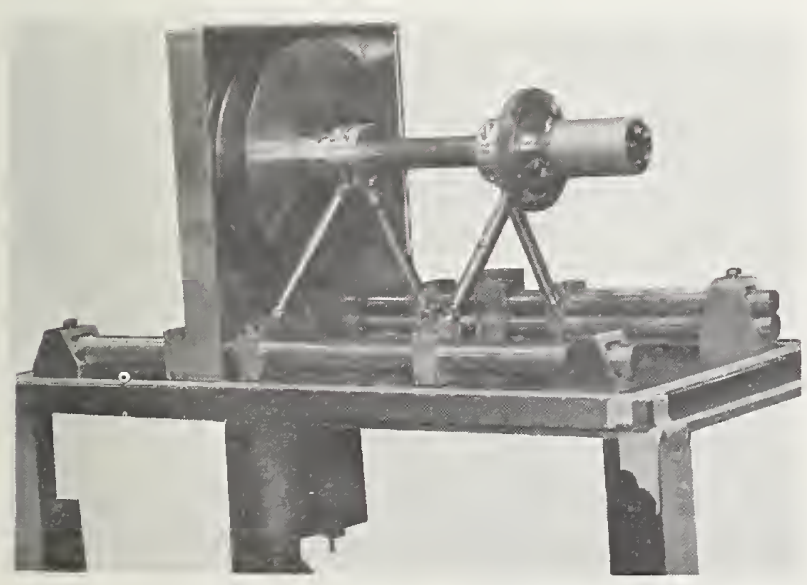

FIGURE 9. The septum, chopping disks, and driving gear, mounted on the three-bar suspension system.

The septum was made from an aluminum alloy and silver plated in the region that is exposed to the electron beam. This is necessary since the oxide la yer on aluminum becomes charged when electrons strike it, thus building up layer on aluminum becomes charged when electrons strike it, thus building up

parallel and immediately adjacent to the septum.
The three-bar suspension system can also be seen in this photograph. The double set of bars was made from $1.91 \mathrm{~cm}$ stainless steel rods, whereas the single bar was $2.54 \mathrm{~cm}$ in diameter. All the elamps used were made from aluminum alloy. to pulse width may be attained, these slits must be rotated at the highest possible speed. In our case they are at the edge of a $20-\mathrm{cm}$ disk revolving at $8,000 \mathrm{rpm}$ giving a surface cutting speed of about $75 \mathrm{~m} / \mathrm{sec}$. This results in a molecular beam pulse which is, as it enters our field of view, $2 \mathrm{~mm}$ long and whose velocity spread is approximately \pm 15 percent of its mean velocity. As each pulse moves across the interaction region, it is viewed at a selected instant by pulsing the electron beam giving a stroboscopic or time-freezing result. A timing reference pulse is generated by means of a light beam which passes through the chopper slit to a photomultiplier (see fig. 10).

Figure 9 shows an overall view of the chopper assembly with its driving motor. The two chopper disks are clearly recognizable on the end of the motor shaft. These disks are mounted with the series of alining pins onto the rotating shaft so that various energies may be selected by changing the slit offset according to the system in section 13.4. The entire chopper assembly, the septum between the two vacuum chambers, the collimating slits, and the Cassegrainian light optical synchronization system are all suspended from the top of the chamber on a three-bar optical bench-type mount. This arrangement allows the various elements to be alined mechanically outside of the vacuum and then clamped rigorously in place, where they will remain despite the inevitable vibration due to the motor. 'The chopper shaft is carried on ball bearings which are lubricated by diffusion-pump vacuum oil. Experience with special vacuum ball bearings, silver lubricated, showed that the standard precision ball bearings, lubricated with vacuum oil, ran much more smoothly and had longer life than the more complicated special-purpose bearings. The slight increase in background pressure, due to the vapor pressure of the oil, seemed to be negligible. The chopper motor is a 400-cycle, three-phase synchronous motor operating from an individual power supply. This motor has proved reliable in operation and is lubricated by the same low-pressure vacuum oil used for the bearings. It is otherwise unmodified and operated in the form that it was received from the manufacturer.

An adjustable mount for a solid target was provided in the interaction space. Two degrees of freedom, permitting the target to be rotated to vary the incidence angle and to line up the target plane with the electron beam axis, were available without breaking vacuum (see fig. 8). The motion was provided by rods driven axially through stainless steel bellows. Aluminum gear linkages translated the axial motion into rotary motion. Although the target mount was not used during the work comprising this report, it was considered necessary to design and build the target mechanism and the synchronization system together so that the separate systems would be geometrically compatible. 


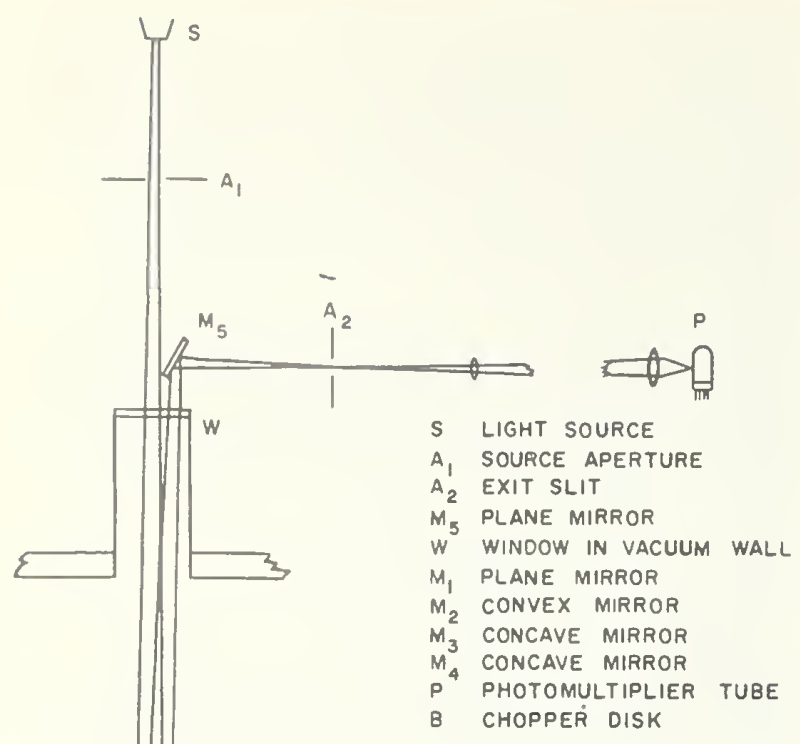

S LIGHT SOURCE

A SOURCE APERTURE

$A_{2}$ EXIT SLIT

$M_{5}$ PLANE MIRROR

W WINOOW IN VACUUM WALL

$M_{1}$ PLANE MIRROR

$M_{2}$ CONVEX MIRROR

$M_{3}$ CONCAVE MIRROR

M. CONCAVE MIRROR

B CHOPPER DISK

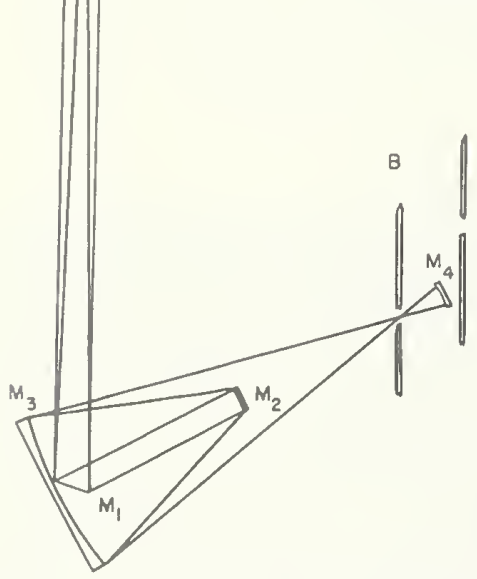

FIguRE 10a. Schematic of the light optics used for synchronizing the electron beam pulse with the molecular beam packet.

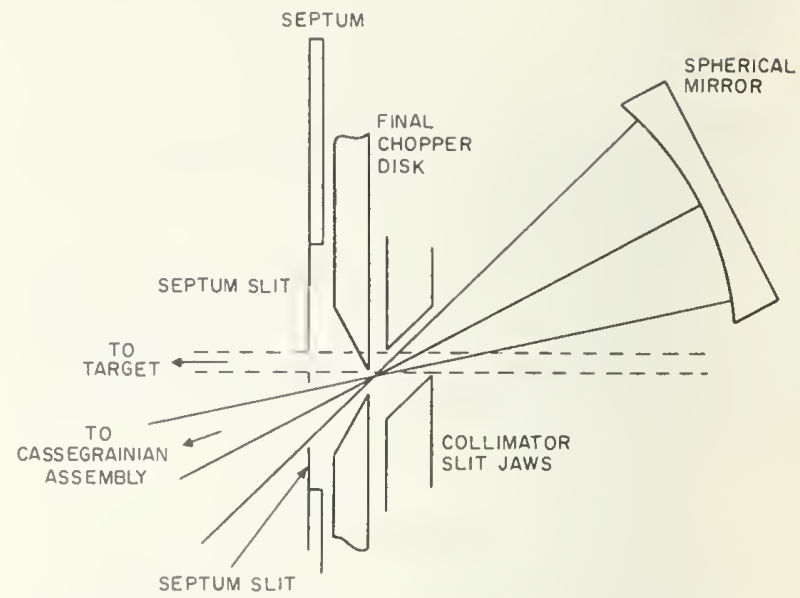

FIgURE 10b. Magnified schematic of the light paths in the vicinity of the chopper blade.

At the instant illustrated in the figure, the atomic beam is still cut of by the chopper, but light is being transmittcd at full intensity. A tomic beam paths, as they will occur a few microseconds later, are shown in broken lines. The light cone is inclined at a $29^{\circ}$ angle to keep the Cassegrainian mirror system (not shown) and the spherical mirror (in the upper right hand corner) out of the atomic beam path. Because of inclination and spread the light out of the atomic beam path. Because of inclination and spread, the light ¿rranged as simple rectangular apertures in plane surfaces. A circular hole in the lower jaw of the septum slit permits passage of light through that surface. The lower jaw of the collimator slit is turned around so that the beam limiting edge of the lower jaw is farther from the chopper disk than is the corresponding edge of the upper jaw. In this way ample passage for the light beam is provided, while the atomic beam is restricted to a width of $1 \mathrm{~mm}$.

\section{Stroboscopic Mechanisms}

\subsection{Optics}

In order that the beam source shall show no significant blurring on the plate, it is essential that the electron beam have an on-time in the neighborhood of 1 to $5 \mu \mathrm{sec}$ and be generated at a time in the range from 10 to $100 \mu$ sec after the time the molecular beam leaves the last chopper blade. When the molecular beam leaves the last chopper blade, a large electrical synchronizing pulse with an adequate time derivative must be supplied.

A light beam modulated by the second chopper disk and fed into a $931 \mathrm{~A}$ photomultiplier provides this timing element. Attempts to use a slit other than the one in the process of cutting the molecular beam proved impractical. Thus we were required to send the light beam and the molecular beam simultaneously through the same slit of the second chopper disk and through the collimating slit.
To accomplish proper synchronization requires some sophisticated optics, since our chopper blades cut the beam at about $75 \mathrm{~m} / \mathrm{sec}$ and a timing accuracy of about $0.2 \mu \mathrm{sec}$ is required. The light signal must increase through most of its range while the chopper blades are advancing only about $0.015 \mathrm{~mm}$. The optics of the system are shown in figures 8 and 10.

The requirement that the light signal should increase substantially while the chopper blade moves $0.015 \mathrm{~mm}$ is roughly equivalent to restricting the light which finally enters the photomultiplier to a slit about $0.03 \mathrm{~mm}$ wide in the plane of the chopper slit. The problem of simply restricting the incident light to a crossover of that size, without regard for intensity, is by no means trivial. The fact that the illuminated area is approximately $0.01 \mathrm{~mm}^{2}$, however, makes the intensity problem also critical. Under ideal conditions, the intensity at the exit of an optical system is $I_{i}=I_{0} \sin ^{2} \alpha_{i}$, where $I_{0}$ and $I_{i}$ are the 
light intensities in the object and image planes and $\alpha_{i}$ is the convergence angle of the light from the last optical element.

Since the light output of a tungsten filament at $2000^{\circ} \mathrm{K}$ is about 250 lumens $/ \mathrm{cm}^{2}$, and $5 \cdot 10^{-6}$ lumens are needed to provide a $1-v$ signal from the photonultiplier, the minimum usable convergence angle is $2 \cdot 10^{-2}$ radians, and a larger angle is to be preferred. Since we cannot place physical mirrors or lenses in the molecular beam path, the light beam must be inclined at a substantial angle with respect to the molecular beam. For obrious reasons, there cannot be a physical aperture limiting the beam spread in the plane of the chopper slit. Thus, the image of the light source or of a suitable set of limiting slits must be focused in a plane which intersects the chopper slit plane at the desired reference position. After the light has passed through the chopper slit into the space between chopper disks, it is in a rery inconvenient place for mounting a photomultiplier. Consequently, it must be transmitted to a more suitable location. One must also aroid contamination of the racuum in the interaction space by a gassy light source and the organic binders of commercial compound lenses. Finally, stray light which might expose the photograph is to be aroided, and the optical elements must not be too sensitive to coating by stray cadmium atoms.

To divorce the problem of adequate illumination in the plane of the chopper slit from that of restricting the area through which the light must pass, we arranged to have an equivalent lens form a magnified image of the chopper slit jaw in a convenient image plane outside the vacuum. Large slits then limit the region for light acceptance as needed. When good focus exists, a sharp magnified image of the chopper blade edge sweeps pass the selector slit. The desired performance is thus obtainable if the resolution of the magnifying system is better than $0.015 \mathrm{~mm}$. A condensing system to provide illumination at the required brightness is still necessary, but stray light in the neighborhood of the desired transmission area does not seriously degrade the output pulse. To aroid contamination of the racuum and loss of performance due to coating of lens elements with stray cadmium, we mounted the source and the photomultiplier outside the racuum chamber and used mirror optics exclusively within the chamber. Instead of being reflected to a separate exit after passing through the chopper slit, the light was returned approximately along its original path by a spherical mirror located between the spinning chopper disks.

The convergent, inclined light beam cannot pass through three parallel horizontal plane slits which limit the molecular beam, since the product of the minimum practicable clistance between slit planes and the tangent of the slope angle greatly exceeds the desired molecular beam width. To bypass this difficulty, we set the reference position of the chopper blarle transmitting the light pulse slightly below the position for full transmission of the molecular beam pulse. The light beam passes through a circular hole in the lower jaw of the septum slit and forms a focused source image in the best plane for passage through the chopper slit. Because the chopper slit is then about $0.050 \mathrm{~cm}$ below alinement with the collimator slit, the light beam easily clears the upper jaw of the collimator slit. The lower jaw of the collimator is set back about $0.5 \mathrm{~cm}$ from the chopper slit plane. Thus, when the light reaches the plane of the lower jaw it has risen well above the jaw and has successfully run the gauntlet of limiting slit systems. The geometry of arrangement of light beam and slits is shown schematically in figures 10a and b.

The object plane and image plane for the spherical mirror is a plane normal to the axis of the light beam, passing through the intersection of the axis with the plane of the chopper disk. The mirror forms an inverted image of the incident illumination pattern at unity magnification. A.light ray may be interrupted by the chopper blade either as it arrives at an object point or as it leaves at a corresponding image point. Thus, no light is returned to the magnifying lens until the chopper jaw is clear of the light axis. The region of incidence for which light transmission occurs then expands in both directions from the axis as the chopper jaw recedes from the axis. The magnifying lens thus sees two shadow images of the chopper slit jaw receding from each other. A magnified image of this phenomenon is projected onto an entrance slit which precedes the photomultiplier. If the system is properly focused, the illumination begins as a line along the middle of this slit and widens until the entire slit is illuminated. The slit jaws thus merely limit the time in which the buildup of light intensity is linear and give the light pulse a flat top after the linear buildup. For maximum freedom from synchronization jitter, the voltage level for triggering the commercial pulse generator should lie at the center of the linear buildup of light intensity. A video amplifier raised the $1-v$ signal level from the photomultiplier to $10 \mathrm{v}$, which is more appropriate for triggering the pulser.

The success of this system depends critically upon the imaging properties of the magnifying lens, which also serves to form an image of the source in the plane of the slit jaws. For this purpose, we used a pair of concentric spherical mirrors arranged as a Cassegrainian telescope objective. Optically, they are equiralent to a strong thin lens located at their common center of curvature. This enables us to obtain a lens of large aperture and high magnification in a position that would not be available for a physical lens. The effective lens position at the center of curvature of the spheres is directly in the path of the electron beam; but the electrons and molecules are not disturbed by a virtual lens. As part of the condenser system, it provides good incident illumination. As part of the viewing system, it provides a highly magnified high resolution image of the chopper slit edge for convenient control of the pulse rise time. By proper choice of the ratio mirror radii, 
we are able to correct spherical abcrration through third order. In an exact ray trace, the deriation from Gaussian image position of a ray converging at $15^{\circ}$ was only $0.025 \mathrm{~mm}$ in the image plane.

The rcmaining details of the optical system are shown in figure 10a. Light emitted by a coiled tungsten filament $S$ passes through a limiting apcrture $A_{1}$ and the planc glass window $W$ into the vacuum system. An image of the filament is projected onto the plane of the chopper slit by the Cassegrainian objective consisting of mirrors $M_{2}$ and $M_{3}$. An angular bend in the optical path is secured by planc mirror $M_{1}$. After reflection by the spherical mirror, the light is again focuscd by the Cassegrainian objective and reflected by $M_{1}$ to pass out of the vacuum system through the plane glass vacuum window $\tilde{H}^{\top}$. The mirror $M_{4}$ is misalined just enough, however, that instead of returning to silt $A_{1}$, the light strikes the small plane mirror $M_{5}$ and forms an imagc of the chopper blades at the exit slits $A_{2}$. The Cassegrainian objective magnifics about 16 times, thus making the adjustment of slits $A_{1}$ and $A_{2}$ relatively easy. Diverting the returning light to a displaced set of slits was possible because the light to and from the filament subtends a small aperture. Thus, the incoming and outgoing light bundles may bc separated in space by a small misalinement. This small aperture is in turn compatible with the requirement of a Jarge aperture for adequate illumination in the chopping plane because of the large aperture ratio (equal to the image magnification) provided by the Cassegrainian objective. After passing through slit $A_{2}$, which limits the height of the light pulse, the light is directed to the photomultiplier by an uncorrected lens. Since this lens is required only to direct all of the light passing through slit $A_{2}$ to the multiplier cathode, it is easily focused and alined. The use of this lens permits us to locate the photomultiplier and associated video amplifier conveniently close to the pulser.

\subsection{Electronics}

The photomultiplier and preamplifier gave pulses of about $10-\mathrm{v}$ amplitude. This was more than sufficient to trigger a delayed pulse generator of commercial manufacture which in turn was coupled by a high-voltage condenser to a diode clamping circuit which controlled the base voltage of the cutoff pulse. A block diagram showing the functions of the various elements in the synchro-

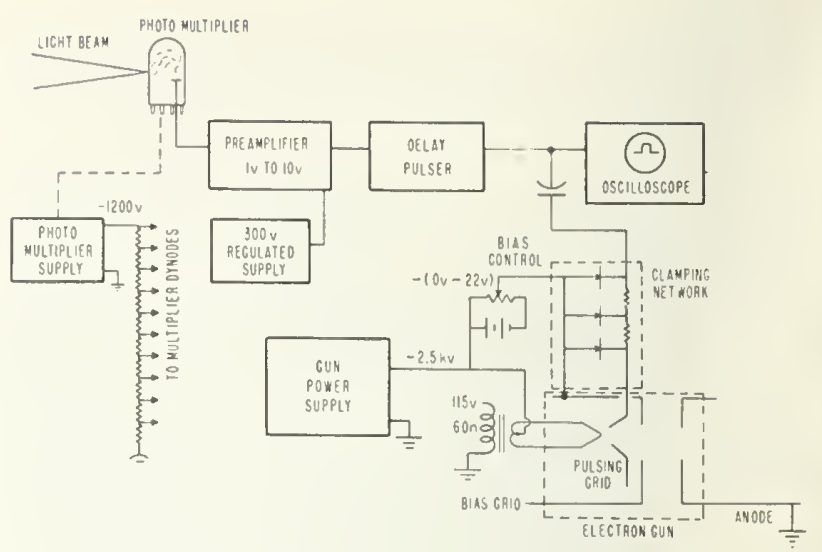

Figure 11. Block diagram of synchronizing elements.

nizing chain is given in figure 11. Although it is convenient and straightforward, this method of turning the electron beam on and off caused some mechanical difficulty in the gun. In order to relieve the pulse generator from having to drive the excessive capacitance of the standard gun, a new gun was constructed in which the control grid was a small element controlled by a conductor running through a separate seal through the side of the gun insulator. In this way, by kecping the grid electrode small and maximizing the clearance between it and the electrodes at fixed potentials, we were able to keep the total capacitance of the control electrode and its lead-in connection to less than $10 \mathrm{pf}$, which is quite acceptable. Prior to the use of this system, extensive experiments were performed in which the beam was deflected electrostatically out of the limiting aperture. For reasons which are still not understood, all these methods gave rise to a residual beam in the off condition which added to the background in the plate. With the electron gun biased below cutoff, there are no electrons anywhere in the optical system and residual effects do not occur. In order to view the molecular beam pulse as it traverses across the viewing space, it is necessary to delay the strobe electron beam by periods of 10 to 100 $\mu$ sec after the chopper blade intercepts the light beam. The commercial equipment proved quite satisfactory for this task, and as the figures show reproducible patterns could be obtained over thousands of exposures where the jitter on the leading edge of the pulse may be deduced to lie well below $2 \mu$ sec.

\section{High-Vacuum Section}

The success of the experiment depends critically upon our ability to maintain a lower density of background gas molecules than the density of chopped-beam molecules in the interaction chamber. We have shown that the signal-to-noise ratio on the photographic plate is roughly the molecular density ratio multiplied by the ratio of clectron path lengths in beam and background. By employing a small aperture at the entrance to the main chamber, we shorten somewhat the effective electron-path length through the background vacuum. An electron may miss the schlieren stop only if the scattering takes place in the region between the entrance aperture and the schlieren stop. If scattering takes place before the electron reaches the entrance aperture, but the electron still goes through that aperture, the electron is definitely captured by the stop. 
This is true because the optical system forms an image of the entrance aperture, at unity magnification, on the schlieren stop, which is twice as large as the entrance aperture. Regardless of any previous scattering it may have undergone, an electron which passes through the entrance aperture is headed for this image on the schlieren stop. Similarly, scattering which takes place after the electrons have passed the schlieren stop is of little consequence, provided that the majority of the electrons is not scattered in that region. The region in which a high vacuum is of crucial importance to the experiment is thus confined to the $75 \mathrm{~cm}$ between the entrance aperture and the schlieren stop.

Conventional vacuum techniques used in construction of electron microscopes are sufficient to provide a vacuum of about $10^{-6}$ Torr. Higher vacua ranging from $10^{-8}$ Torr to $10^{-10}$ Torr are possible today by rigorous exclusion of organic substances from the racuum chamber, driving off adsorbed gases through baking, and gettering, or cryogenic pumping [44]. The design adopted in this experiment was a compromise between the need for high vacuum and reasonable economy in construction. The optical sections containing the electron gun and the photographic plates were separated from the interaction space by airtight walls pierced only by the $1-\mathrm{mm}$ entrance aperture and the $3-\mathrm{mm}$ aperture which surrounds the schlieren stop. The molecular beam source, the chopper motor, and the chopper disks are also inherently gassy. The need to minimize distance between the chopper and the field of view made it impractical to put these elements in a separate chamber from the interaction space. Instead, a thin, stainless steel septum was used to divide the chamber into two separately pumped sections (see fig. 9). Adjustable slits on the septum reduced the area for molecular communication between the two subchambers to the minimum that could be achieved without having the septum slits define the molecular beam. The arrangements for support and removal of the septum were not considered sufficiently reliable to depend upon the septum slits for beam collimation. The septum slit width was thus set at $1 \mathrm{~mm}$ to give reasonable running clearance around the molecular beam.

The apparatus is thus divided into four separately pumped chambers, $A, B, C$, and $D$, as shown in figure 2. Sections $A$ and $B$ are housed in a rectangular, stainless steel chamber $25.4 \mathrm{~cm}$ wide and deep by $51 \mathrm{~cm}$ long, divided into two sections by the septum which slides into close-fitting grooves in the walls of the chamber. By providing a series of grooves, we made the geometry of the chambers easy to vary. All of the apparatus required in these two sections is hung from a three-bar optical bench attached to the roof plate, which is removable. Thus, optical alineinent of the various parts may be carried out in the atmosphere, in full view, without the spatial restrictions of the chamber walls. The alined system is then simply lowered into the rest of the chamber, which merely serves as the pumping orifice and racuum envelope. The two sections of the chamber are pumped by separate 6-in. oil-diffusion pumps with individual speeds of 900 liters/sec. Liquid-nitrogen-cooled baffles keep to a minimum the amount of oil vapor diffusing back into the chambers. Drift tubes $20 \mathrm{~cm}$ long to the entrance aperture and the schlieren stop form an integral part of the main chamber $A$. All of the removable flanges connected to the main chamber employ flat, soft aluminum gaskets. A seal is accomplished by opposing $V$-shaped, stainless steel ridges which bite into the metal from both sides. The ridges are integral parts of the respective mating flanges. Two sets of ridges are employed, one inside the other, so that air must diffuse through leaks in both sets of ridges
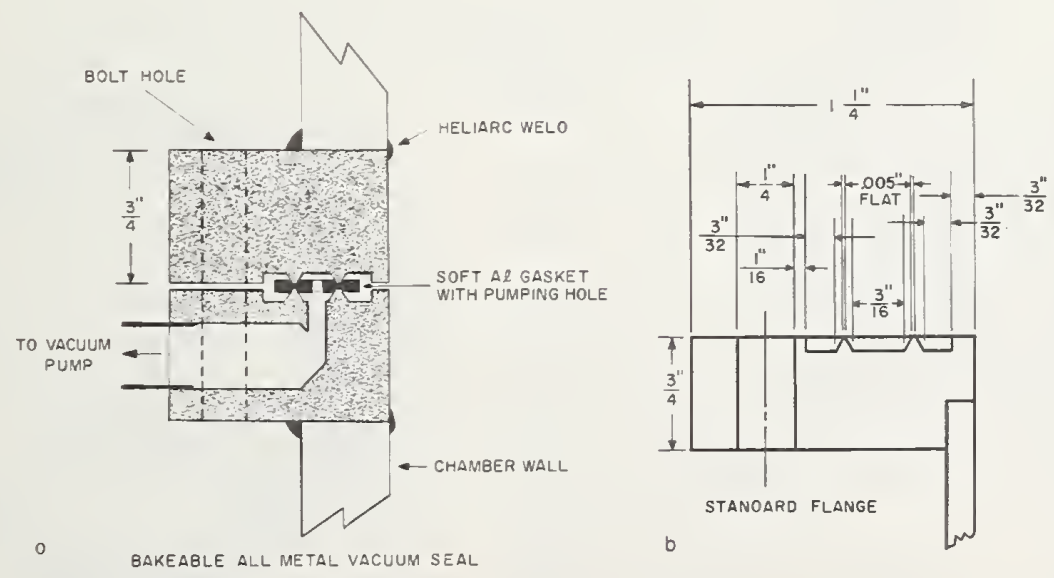

FIGURE 12. Cross-sectional view of the all-metal bakeable seal used on all parts of the apparatus that were heated.

The figure on the right shows the dimensions that were used. The dimensions are not critical and small variations are tolerable. We found that one stringent requirement on this type of seal is that the 0.005 -in. flat on the ridges must be completely Iree from all scratches, nicks, or other rough spots. The principle behind this type of seal is that as the ridge is slowly squeezed into the gasket material the metal in the gasket is smoothly displaced about the ridge.
On the spot where a nick or scratch is present, the metal will not displace smoothly but will instead tear leaving a minute hole which acts as a very slow leak. We used soft aluminum gaskets, but copper or other soft bakeable metals are also suitable. 
to enter the vacuum system (see fig. 12). The space between the inner and outer seals is evacuated to about $100 \mu$ pressure by being connected to the fore-pump line. Thus, a perfect seal is not required to keep the leak rate well within our pumping capacity. In practice, however, these seals held so well that only a slight increase of pressure was encountered if the space between seals was opened to the atmosphere. Thus, the extra seal, with pumping between seals, insured that the leak rate past the seals had to be a negligible source of gas in the interaction chamber. In the event of a suspected gasket leak, the space between seals was filled with helium and the vacuum leak tested with a commercial helium leak detector, thus providing a quick means of finding the offending flanges.

With all of these precautions and with all of the gas sources removed, however, the equilibrium pressure of the interaction chamber $A$ registered about $3 \cdot 10^{-7}$ Torr, which is definitely not good enough for chopped-beam work. To give the chamber $A$ a more decisive advantage, vacuumwise, over the other chambers, we resorted to bakeout at temperatures from 150 to $300{ }^{\circ} \mathrm{C}$, thus removing adsorbed material from the walls of the chamber. To insure the persistence of high pumping speed, untroubled by back diffusion at the lowest pressures, we provided for sealing off the oil pump with a 6 -in. gate valve and added a cryogenic trap. With the pump sealed off, liquid hydrogen or liquid helium is added to the trap, and all of the heavier gases are condensed and frozen on the cold surfaces. Because of its relatively low heat of vaporization and high cost, liquid helium cannot be economically used in Dewars where the cold surfaces are exposed to room temperature thermal radiation. Thus, communication between the liquid helium tank and the chamber to be pumped must pass through a one-hit baffle, and all straight-line optical paths between the tank and surfaces above liquid helium temperature must be eliminated.

The design of our cryogenic trap is shown in figure 13. A C-shaped liquid nitrogen tank surrounds the cryogenic section, and a single row of copper venetian blinds at liquid nitrogen temperature is arranged so that all incoming radiation paths miss the inner tank, while the pumping impedance is held to a minimum. The evaporated helium or hydrogen passes through a loosely wound spiral coil surrounding the inner tank before being conducted to the atmosphere. From the side facing the opening of the C, gas molecules can pass between the coils to reach the tank, and they can also reach the tank in a straight line path from the bottom of the cold shield. On the sides facing the solid wall at liquid nitrogen temperature, however, the space between coils is filled by a mantle made of aluminum foil to which the coils are attached.

\section{OF 3 TUUBES SUPPORTING LIQUID HELIUM FILLING TUBE \\ THE LIQUID N2 SHIELD}

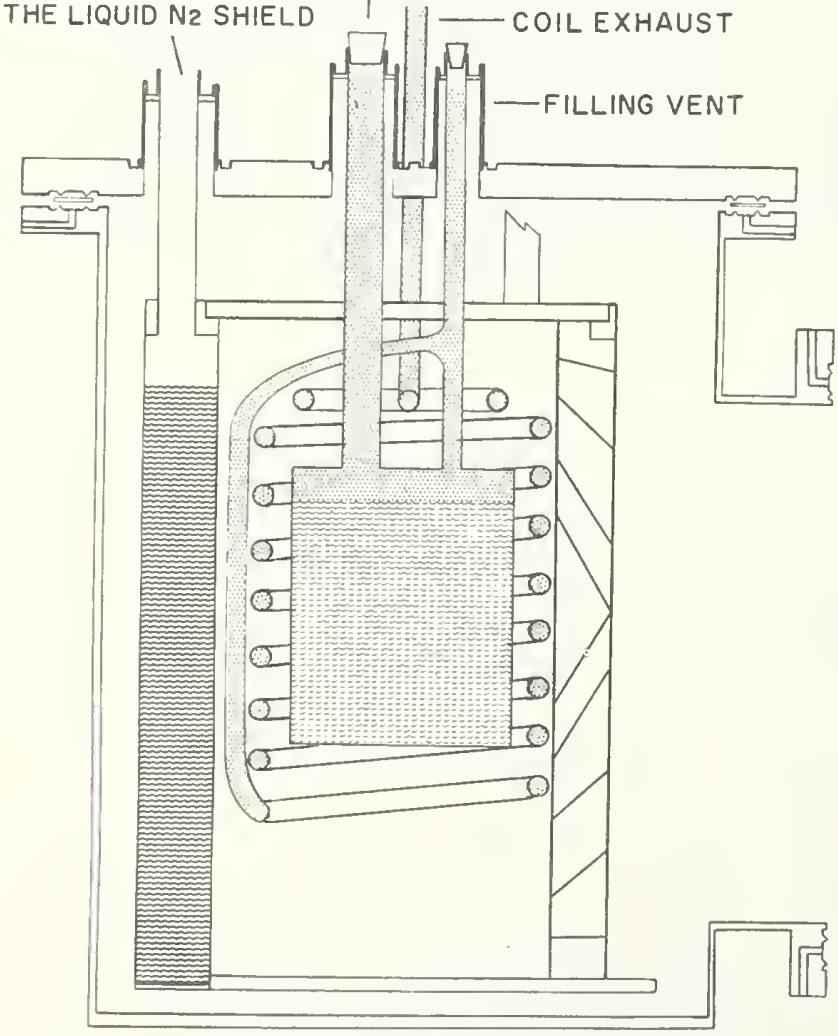

LIQUID HELIUM TRAP

FIGURE 13. Drawing of the liquid helium trap. 
The coils and the mantle are ccoled to a temperature well below that of liquid nitrogen by the cold gas evaporating from the liquid state. This is possible because, in the case of helium, the heat required to raise the gas temperature by even $10^{\circ} \mathrm{C}$ is $\mathrm{far}^{\circ}$ greater than the heat of vaporization from the liquid state. Thus without appreciably lowering the pumping speed of the Dewar, the coil and mantle reduce still further the thermal radiation available to evaporate the helium or hydrogen. In this connection it is worth noting that lowering the temperature of a surface by a factor of 2 lowers the rate of blackbody radiation br $2^{4}$, which is a good order of magnitude. About 2.1 liters of liquid helium are used in filling the Dewar, which has a capacity of about 1 liter. One filling is sufficient for an 8-hr day of operation. Tith either liquid hydrogen or liquid helium, the Dewar was successful in producing a $10^{-8}$ Torr vacuum with the apparatus actually taking pictures of chopped molecular beams. Hydrogen was slightly favored because of its lower cost.
The chamber housing sections $A$ and $B$ are fabricated by shielded arc welding of $3 / 4$-in. plate of 304 stainless steel. Strip heaters are bolted onto the outside of the walls, and the chamber is surrounded by asbestos sheets wrapped in aluminum foil for bakeout. The bakeout power consumption was $2800 \mathrm{w}$. The focusing coils were slid away from the main chamber for bakeout. Other precautions were to surround the oven and the chopper motor with coppe: shields which could be cooled to liquid nitrogen temperature. Thus, it was possible to bake the chamber at temperatures up to $300{ }^{\circ} \mathrm{C}$ without overheating the chopper motor or evaporating the cadmium from the oven. Nevertheless, the motor was the delicate component whose presence limited baking temperatures. Since this apparatus was built, motors with the ability to withstand higher baking temperatures have been produced commercially. With the higher bakeout temperature and with an extra stage of differential pumping, it is believed that a $10^{-10}$ Torr vacuum should be possible in instruments of the future.

\section{Intermediate-Vacuum Section}

The motor coils, motor insulating materials, bearing oils, and the hot surfaces of the oren, its shields and mounts, together. with the air dissolved in the cadmium, provide copious sources of gas in chamber $D$. The tight-fitting septum with a total area open to section $A$ of less than $0.5 \mathrm{~cm}$ and the narrow clearance between the chopper disk and the septum combine to reduce effective pumping speed between the two chambers to a few liters/sec. In the present design, the approach to limiting the resulting throughput of gas into the main chamber was limited to brute force pumping with a 900 liters/sec oil-diffusion pump. For ultrahigh vacua, it is recommended that this section be modified to provide a separately pumped intermediate chamber between the gassy components and the interaction chamber $A$. This would require moving the oven about $3 \mathrm{~cm}$ back from the chopper disks and inserting a second septum into the main chamber. The only gassy component left in the intermediate chamber would then be the bearing for the chopper drive shaft. By means of close clearances between a rotating sleeve attached to the chopper disk and the main support shaft, the rate of leakage to the intermediate chamber could be kept low.

The electron gun, section $B$, and the acceleratorcamera, section $C$, are likewise rather gassy. The hot filament gun warms adjacent parts to a point where they give off adsorbed gas continuously, but not to a point where the evolution of gas is completed in any reasonable time. In the present experiment, the $1 \mathrm{~mm}$ connecting aperture between this chamber and chamber $A$, limiting pumping conductance to less than 0.1 liter/sec, was sufficient to make chamber $B$ a negligible source of gas in the main chamber. Brass con- struction with silver-soldered joints and elastomer $\mathrm{O}$-ring seals was adequate for this section, and also for section $D$, since the vacuum requirements are not stringent. Both of these systems were pumped by MC-275 oil-diffusion pumps with a rated speed of 270 liters/sec. These $4-i n$. pumps were suspended underneath 8-in. baffle chambers which provided a 4-in. cylindrical passage with cold walls. At the entrance to this liquid nitrogen cooled passage, a cooled one-hit baffle consisting of truncated conical shells of copper provided a barrier to oil molecules with minimum pumping impedance. The precaution of providing a ring aperture with a sharp edge just below the cryogenic tank appears to have been effective in preventing creep of oil up the walls of the baffle. No traces of oil were ever observed in either the gun section or the camera section.

In the camera, section $C$, the moisture and adsorbed gas on the emulsions provide serious contamination. The annular space between the schlieren stop and the surrounding aperture has five times the area of the entrance aperture. Thus, the flux of gas from the camera section to section $A$ is probably an important source of the background gas in that chamber. Since there is no way to eliminate effusion of gas from the photographic plates, it appears that the best way to reduce this gas source is to install an extra stage of differential pumping. A drift tube with an attached purnp, a magnetic lens, and an aperture of the same size as the schlieren aperture would be the extra equipment required. The intermediate stage could then also be subjected to baking if desired. With this added stage of differential pumping, leakage into the main chamber could be reduced by a factor of at least 100 . 


\section{Vacuum Pump System}

The four diffusion pumps pumping the separate regions are connected to a commion manifold and to a 600-liters/sec booster pump. The fore vacuum for this pump is provided by a 7.1liters/sec mechanical pump. In order to isolate its vibration, the mechanical pump is placed in an adjoining room. Throughout the course of the project, this pumping system has proved reasonably trouble-free and, in general, satisfactory.

\section{Auxiliary Equipment}

Other prominent features in the photograph of the overall experimental arrangement, figure 14 , are the four rectangular coils used to counteract the earth's magnetic field. These coils, generating two components separately variable to counteract the earth's field, are in the form of elongated Helmholtz coils and give a compensation which is accurate to perhaps 10 percent. The details of this field's compensation are given in section 13.2.

These coils, together with the three magnetic lenses in the system, are energized from electronically regulated, constant-voltage sources. It was found that the drifts due to the change of resistance of the coils were small enough that the added expense and complexity of constant current supplies were unnecessary. The following table gives the characteristics of the power supplies used to excite these various coils and to provide the electrostatic potentials needed for the electron optics.
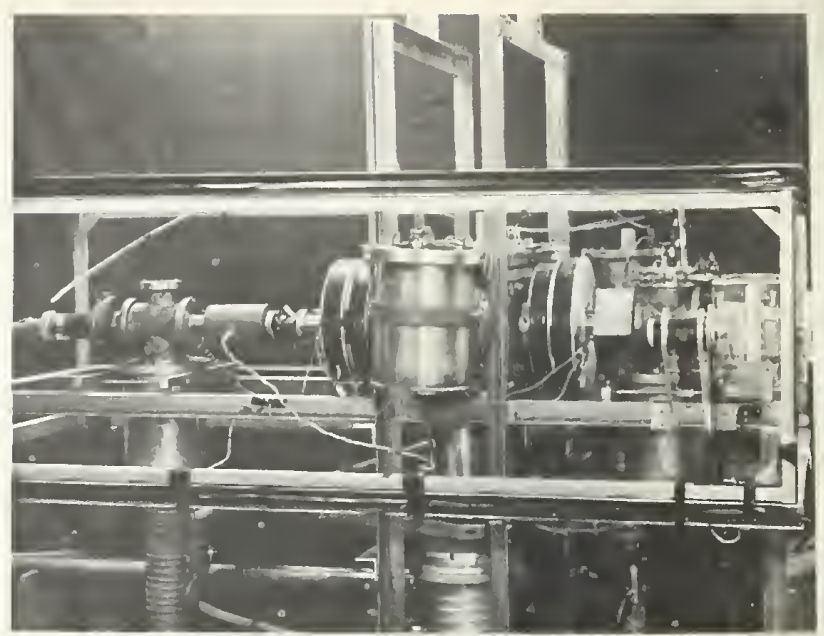

FIgURE 14. Overall view of the assembled apparatus.

The electron optical axis is from left to right, whereas the molecular beam axis is directed out of the paper toward the readcr. All of the components are described in the text except the three-way glass valve which is at tached to the camera. This is a commercial object which was used to pre-pump the camera chamber and seal it off before it was opened to the varuum in th: apparatus. One can see scme of the strip heaters attaehed to the cryotrap and main chamber in preparation for a baking eycle.

Characteristics of power supplies

\begin{tabular}{|c|c|c|c|}
\hline Function & Potential & Current & Regulation* \\
\hline Gun.- & 0 to $6 \mathrm{kV}$ & $\stackrel{m a}{0 \text { to } 100}$ & $\%$ \\
\hline Condenser......... & 0 to $500 \mathrm{v}$ & 0 to 500 & 0.01 \\
\hline Focusing coils_. & 0 to $300 \mathrm{v}$ & 0 to 300 & 0.02 \\
\hline Post acceleration... & 0 to $30 \mathrm{kv}$ & 0 to 10 & Unregulated \\
\hline Post deceleration. & 0 to $300 \mathrm{v}$ & 0 to 100 & 1 \\
\hline Magnetic compensation twin & 0 to 1008 & 0 to 100 & \\
\hline $\begin{array}{l}\text { suppres } \\
\text { Pulse amplifier. }\end{array}$ & $\begin{array}{l}0 \text { to } 100 \mathrm{~V} \\
0 \text { to } 300 \mathrm{~V}\end{array}$ & 0 to 150 & $\begin{array}{l}0.1 \\
0.02\end{array}$ \\
\hline
\end{tabular}

*All regulated supplies supply constant voltage.

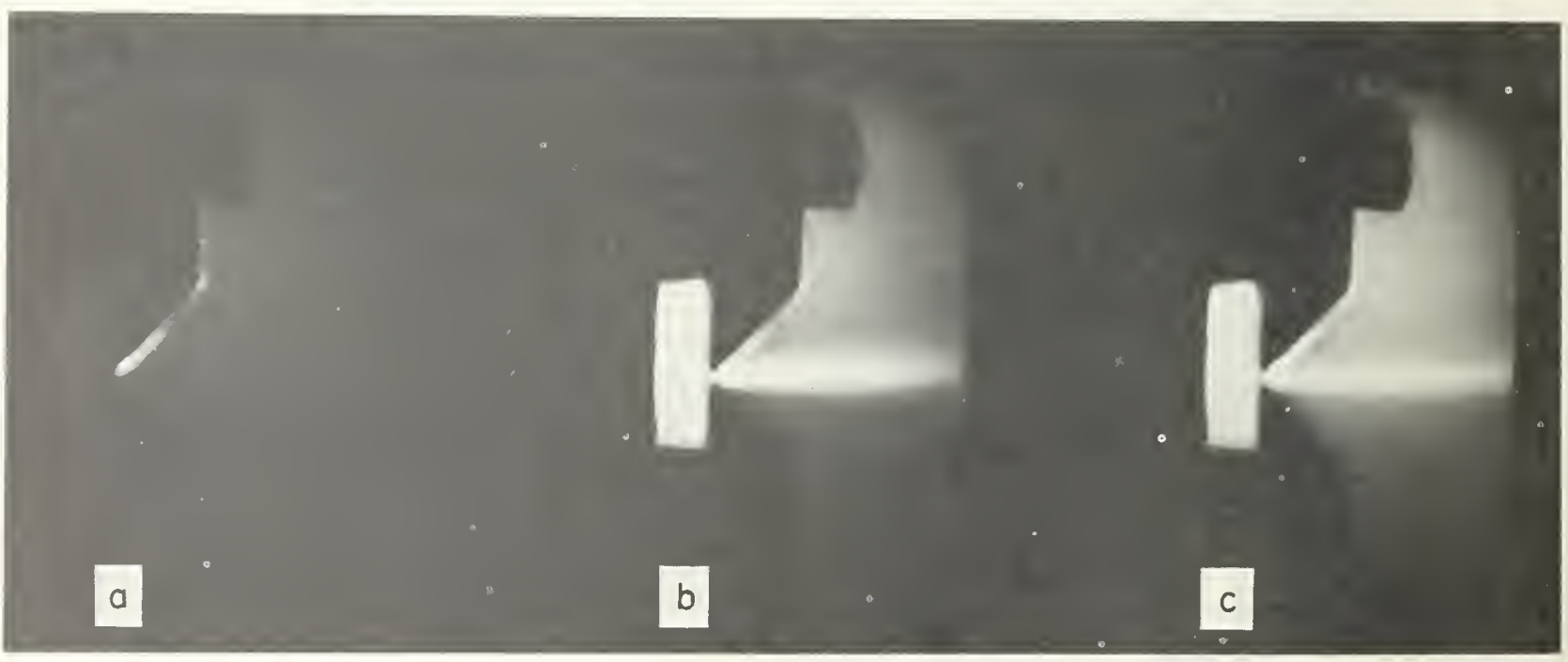

Figure 15. Three electron schlieren images of continuous atomic beams.

To get the proper perspcetive, one must visualize the electron beam propagating from behind the paper and forming an image in the plane of the photo.

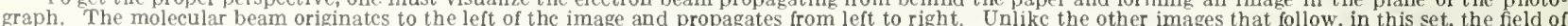

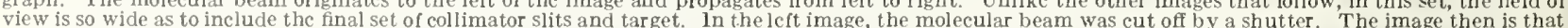

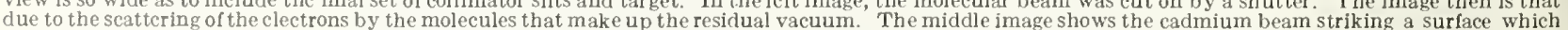

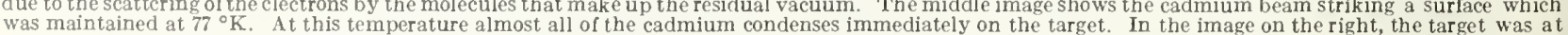

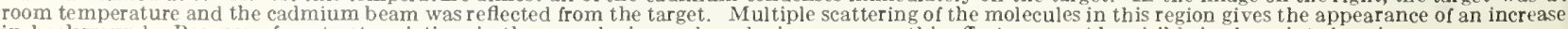
in background. Because of contrast variations in the reproducing and producing processes this effect may not be $\nabla$ isible in the printed copies. 


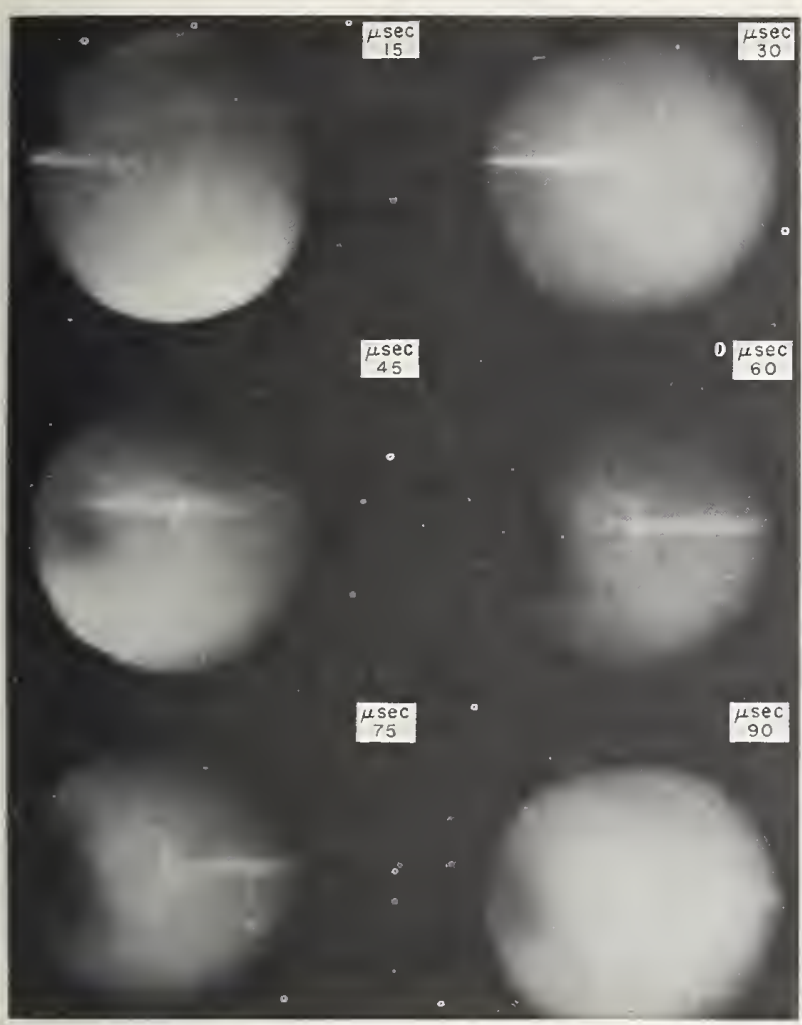

FIgURE 16. Stroboscopic images of chopped atomic beams.

Set of images produced by operating the instrument stroboscopically. In contrast with the previous figure, the field of view here is restricted to exclude the collimator and target areas. Starting with a $15 \mu \mathrm{sec}$ delay from exclude the collimator and target areas. Starting with a $15 \mu$ sec delay from the time the pulse is generated until the on time for the electron beam pulse, With each successive image representing an additional $15 \mu$ sec delay, one can With each successive image representing an additional $15 \mu \mathrm{sec}$ delay, one can
see the molecular packet progressing across and out of the field of view. If
one examines the form of the packet, one sees a taper in the trailing end, i.e., one examines the form of the packet, one sees a taper in the trailing end, i.e., continue to stream past the edge of the chopper slit as it is in the process of cutting off the beam segment from one side. A similar taper should appear in the leading end.

The visibility of both of these tapers is blurred, however, by the spread in molecular velocities over a 3 to 2 range. In the trailing edge of the pulse this velocity spread is effective for a shorter period of time than in the leading edge. Hence, the effect of the spread almost completely washes out the edge. Hence, the efect of the spile this taper is clearly visible in the trailing edge. The slope of this taper is the ratio of chopper cutting speed to mean beam velocity, i.e., 1 to 4 . The 3 to 2 spread in molecular velocities
produces some increase in the length of the packet as it proceeds across the field of view.

These images have a time resolution, i.e., and electron pulse width of 3 usec. The maximum particle density is about $2 \times 10^{10} / \mathrm{cm}^{3} . \mathrm{cm}^{3}$.

The irregular bright spot in the center of the schlieren image is an imperfection of the electron optical imaging technique.

\section{Experimental Results}

The preceding three figures give the kind of pictures which may be obtained with this equipment. Figures $15 \mathrm{a}, 15 \mathrm{~b}$, and $15 \mathrm{c}$ are photographs of a continuous cadmium beam reflected from a brass surface and diffusing into space. The beam contains a significant density of multiply reflected atoms. The latter two figures show the main results with a sharp cadmium beam. Figure 16 shows the spread of the cadmium beam pulse due to the finite energy resolution of the chopper slit as the pulse progresses across the observation chamber. In all cases, one can notice certain artifacts in the background which are described in the caption. By the end of the program, the sources of most of these were understood and the

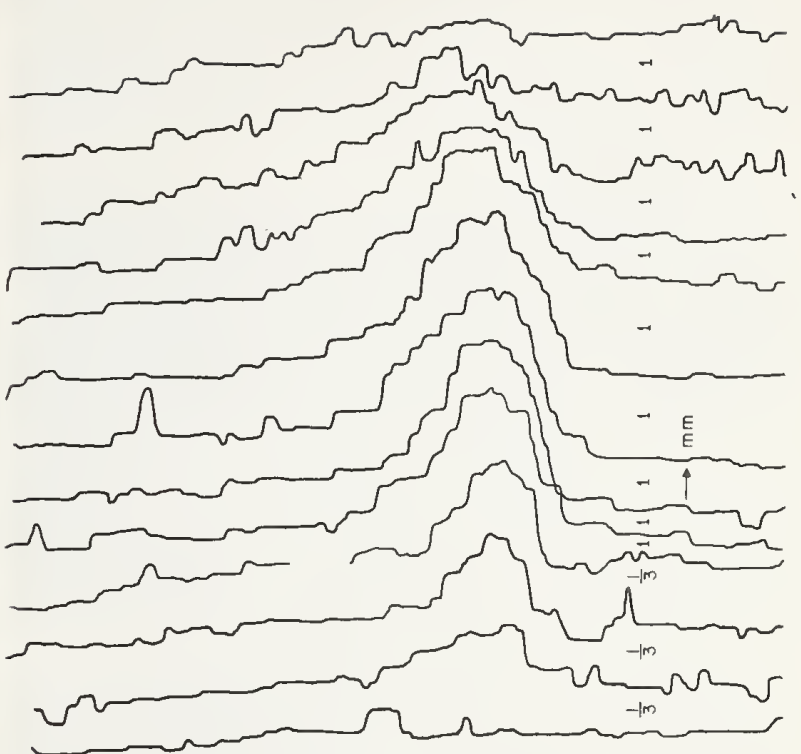

FIGURE 17. A series of microdensitometer traces across the image labeled $30 \mathrm{\mu sec}$.

The vertical scale represents the position along the length of the packet in $\mathrm{mm}$ units. The lowest trace is that of the background at the edge of the field of view on the left side of the image. The successive traces show the rapid build up and one can also see the taper and spreading in the packet rapid build up and one can also see the taper and spreading in the packet. By subtracting the background in the image, it appears that the photographic
density difference can be read with an accuracy better than 10 percent, and density difference can be read with an accuracy better than 10 p
spatial resolution better than $0.1 \mathrm{~mm}$ on the photographic plate.

most serious of these disturbing factors removed. How these factors affect the available contrast is shown in figure 17 , which presents densitometer traces under various conditions of alinement.

\section{Interpretation of the Data}

We have shown that a practical electron optical schlieren apparatus does produce photographs of low-density gas formations with a spatial resolution of about $0.1 \mathrm{~mm}$ and a time resolution of about $1 \mu$ sec. The principle of velocity analysis, using data from these photographs, is essentially that if a group of molecules is in a fixed point at time $t_{1}$, then their spatial distribution at time $t_{2}$ is readily translated into the velocity distribution. More precisely, if we choose our coordinate system so that all molecules are at the origin at time $t_{1}$, and their spatial distribution at time $t_{2}$ is given by $N(x, y)$, then the velocity distribution is given by

$$
\ell\left(V_{x}, V_{y}\right)=\left(t_{2}-t_{1}\right)^{2} \cdot N\left(V_{x}\left[t_{2}-t_{1}\right], V_{y}\left[t_{2}-t_{1}\right]\right) \text {. }
$$

Experimentally, however, it is impractical to use a molecular beam packet whose initial dimensions are negligible in comparison with its final dimensions. As the packet spreads in space, its 
mean density decreases correspondingly until it is masked by the background gas density. Whatever velocity distribution information is to be obtrined must be extracted from photographs in which the mean spread due to the different velocities amounts to only a few times the original beam dimensions.

The original distribution of the beam packet may take either of two forms, depending upon the purpose of the experiment. We shall treat both initial forms by means of a single mathematical formalism. The first case to be considered is the determination of velocity distribution from two photographs of the same beam packet at different times. We will concern ourselves only with the special case in which the velocity distributions of molecules at different positions in the earlier picture are assumed identical. This condition is approached in the case of a beam packet which has just left the second chopper. We then have an initial spatial distribution $N_{t_{1}}(x, y)$.

The second case which we shall consider is the determination of the velocity distribution of molecules which have been reflected from a solid target. For this case we choose the Cartesian coordinate system so that $y=0$ on the target surface. We can then write a space-time density function $N_{0}(x, t)$ describing the arrival of molecules at the target. The form of $N_{0}$ might be determined from a series of stroboscopic pictures showing the progress of the incident beam toward the target surface or, alternatively, it might be determined by calculation from the known geometry of the experiment. In section 13.4 we discuss the calculation of $N_{t_{1}}(x, y)$ and of $N_{0}(x, t)$.

Assuming that this initial solution is available, one has first the task of determining the spatial distribution $N_{t_{2}}(x, y)$ of the molecules from the density pattern on the photograph and then the task of deducing the velocity distribution from the initial and final molecular distributions.

\subsection{Scanning of the Negatives}

A distribution $N_{e}\left(x_{i}, y_{i}\right)$ of electrons on the photographic plate gives rise to a photographic density distribution $D\left(x_{i}, y_{i}\right)$ which may be read with a photodensitometer. Here, in accordance with usage in photographic work, $D$ is the base 10 logarithm of the ratio of light transmitted through the developed negative to that incident upon the negative at each point. Present-day evidence is that for electrons, $D$ is proportional to $N_{e}$ at low exposure levels, although the curve is certainly nonlinear at higher levels. This behavior contrasts sharply with the situation in light optics, where $D$ is usually plotted against the logarithm of the intensity. In that case, a threshold level of illumination is found, below which the response is relatively poor.

The absence of a threshold in the case of exposure by electrons may be understood from the fact that each $10-\mathrm{kev}$ electron has sufficient energy to expose several grains of the emulsion, while several light photons are required to expose a single grain. At low exposure levels, the majority of photons may be involved in grain excitations which did not produce exposure, and the response at low levels is thus poor. In electron exposure, however, the first electron is as effective in exposing grains as any other electron. Each developed grain of silver absorbs a fraction of the light incident on it which is proportional to its thickness. When a light ray passes through a series of developed grains, the successive losses by absorption produce an exponential dependence of transmitted light on the number of grains encountered. The photographic density, which is proportional to the logarithm of the transmitted light, is thus directly proportional to the spatial density of developed grains.

If the electron beam energy is sufficient to penetrate the emulsion with most of the incident energy intact, the probability that a silver grain will be developed is independent of its depth in the emulsion. The probability that a silver grain will escape excitation then becomes a decreasing exponential function of the number of incident electrons per unit area in the vicinity of the grain. Combining these results, we conclude that the photographic density must be related to the number of electrons per unit area by an equation of the form

$$
D\left(N_{e}\right)=D_{\max }\left[1-\exp -\left(\frac{N}{N_{0}}\right)\right]
$$

where $D_{\max }$ and $N_{0}$ are constants characteristic of the emulsion, the developer, the development time, and the development temperature.

Instead of depending upon the accuracy of this formula, however, it is best to calibrate the plate response against known relative electron densities. We are not much concerned with absolute electron densities, since we intend to calculate only a relative density pattern for the molecular beam packet.

To calibrate the emúlsion, following a method widely used for calibration of spectroscopic plates [45], we set up our apparatus in the schlieren condition and deliberately bias the electron gun to provide a non-uniform illumination pattern. The situation is ideal if the beam intensity fades gradually to zero at the edge of the field of view. A series of plates is then exposed with linearly increasing exposure times, and a densitometer trace is taken along a common line on each exposure. The instrument must be adjusted to give all of these traces with respect to a common base line. When these traces are superimposed, we obtain a picture something like figure 18 .

Starting at any convenient point on the lowest curve, we define a unit of electron density as the density required to produce the photographic density of that point on the lowest curve. The photographic density corresponding to $2,3,4$. . units of electron density may then be obtained by drawing a vertical line through the upper curves and picking off the ordinates. We plot the resulting 

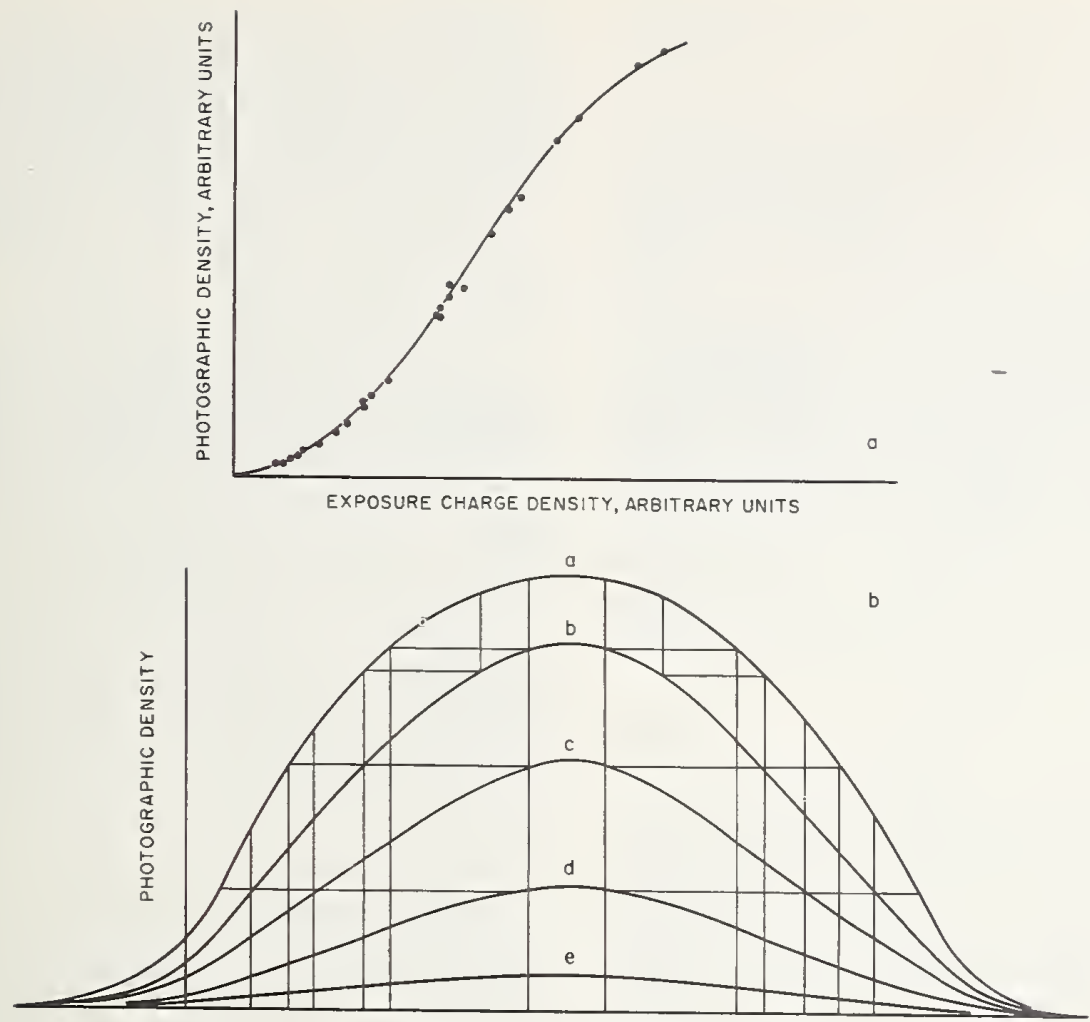

POSITION, SCALE IS ARBITRARY

FIGURE 18. Illustration of the method for calibrating photographic plate response.

To illustrate the method, we assumed a hypothetical photographic response curve, shown as the solid curve in figure 18a. Aside from being monotonic,

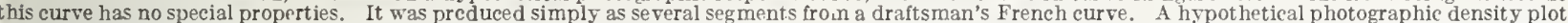

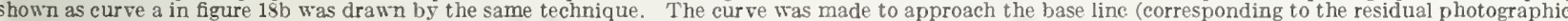

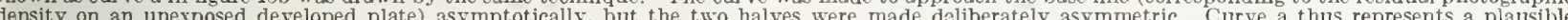

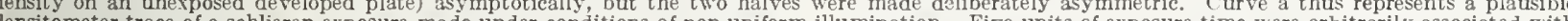

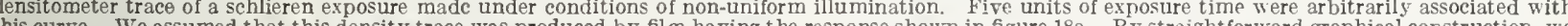

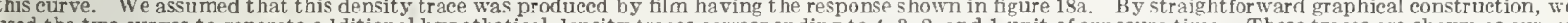

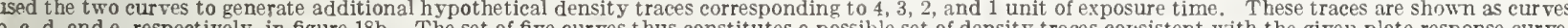

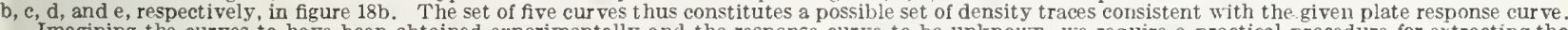

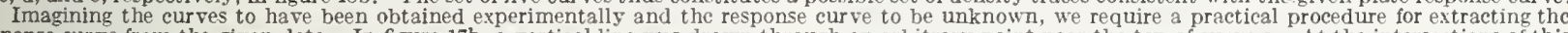

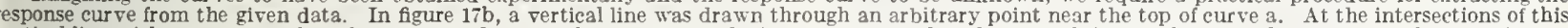

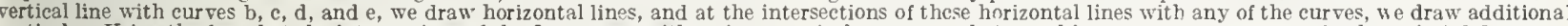

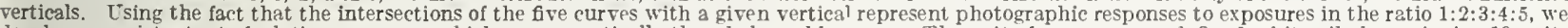

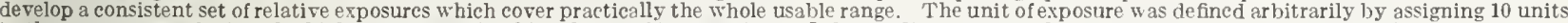

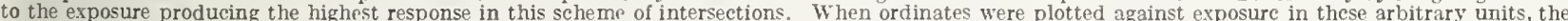

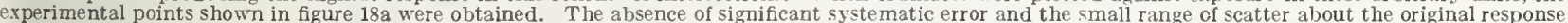
curve show that the method can produce accurate calibrations, even when the plate response is highly nonlinear.

The horizontal scale used in the plotting of points in figure 18 wen the plate response is highly nonlinear.

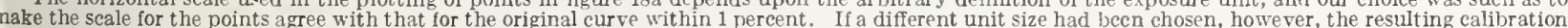

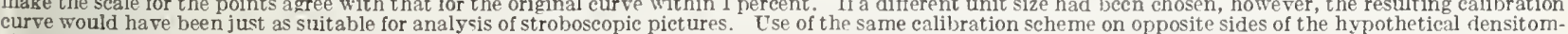

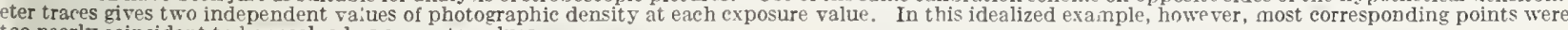
too nearly coincident to be resolved as separate values.

densities against units of electron density. Now from the intersections of the vertical line with the upper curves, we draw horizontal lines and note where these intersect any of the curves. Wherever this happens, we can draw another vertical line and find the density readings corresponding to another series of integral multiples of the unit of electron density. All of the information thus obtainable by continuing the series of horizontal and vertical lines and deducing the number of exposure units that should be associated with each intersection of a vertical line with a curve is plotted on a single graph. The points thus obtained should include most of the integral multiples of the basic exposure unit.

Next, we can start with another arbitrary point and construct a graph of photographic density versus integral multiples of the second arbitrary exposure unit. After drawing smooth curves through the two sets of points, we then.attempt to reconcile the exposures which produce equal densities. This process gives a highly redundant way to compute the ratio of the two arbitrary exposure units. The two curves may then be combined into a single curve with twice as many experimental points. Continuing in this manner, we can obtain as many points as desired for a precise calibration curve. The technique works equally well whether the response is linear or not.

We can use the calibration curve for our emulsion to translate photographic density patterns into corresponding electron density patterns through point-by-point conversion.

Suppose now that we have two electron schlieren photographs taken under identical conditions except for the time delay between the light signal 
and the electron beam pulse. In the first exposure, the time delay is set at perhaps $1000 \mu \mathrm{sec}$, so that the moleeular beam pulse, whether direct or reflected, has been completely dispersed and trapped. This pieture gives a map of the product of incident electron current density and background gas density. Since we expect the background gas density at this late point in the duty cycle to be essentially uniform, we can attribute all of the density variation to irregularities in incident current density and in instrument response (due to lens aberrations, nonparallel beam, etc.). In the second exposure, we have the same background plus the atomic beam packet at the time which we have selected.

If the two sets of exposure conditions were exactly the same, we can write the molecular density function as seen in the image plane

$$
D\left(x_{i}, y_{i}\right)=\frac{N_{2}\left(x_{i}, y_{i}\right)}{N_{1}\left(x_{i}, y_{i}\right)}-1 \text {, }
$$

where $N_{1}$ and $N_{2}$ are the observed electron densities in the respective exposures. If through imperfect control of exposure conditions, the integral of gun current times time in the two exposures is not the same, the constant in eq (12.3) will differ from one. In the absence of any better criterion, we would be forced to set this constant in the manner which gives the most plausible spatial distribution of the chopped pulse.

Finally, the density function computed in the image plane is transferred to the object plane by substituting $\left(\frac{x_{0}}{N}, \frac{y_{0}}{N}\right)$ for $\left(x_{\imath}, y_{i}\right)$. The density function thus computed is expressed in units of the background density multiplied by the ratio of effective electron path lengths in the background and in the beam.

\subsection{Computation of Velocity Distributions}

The method of computation employed in this section is similar to an iteration method used by Bennett and Estermann [46] in a one-dimensional study of the velocity distribution in a molecular beam. In the present work, this method is adapted to a different form of input data and extended to cover two-dimensional distributions in velocity space.

We assume that by means of the densitometer the photographic density distribution $D\left(x_{i}, y_{i}\right)$ has been determined and that the procedure of section 12.1 has been carried out, yielding the spatial density function $\ell^{\prime}\left(x_{0}, y_{0}\right)$, which is the line integral of the molecular density function along the electron path through $\left(x_{0}, y_{0}\right)$. For simplicity, in this section, we drop the subscript o, which has been used to indicate merely that the coordinates are measured in the object plane. It is important at this point that the orientation of the coordinate axes with respect to the molecular beam and to the target plane should be specified unambiguously. In the work which follows, the $x$-axis is parallel to the target plane and directed upward, while the $y$-axis is directed horizontally in the direction of propagation of the atomic beam. The origin is loeated in the horizontal mid-plane of the atomic beam at its intersection with the plane of the final beam chopper. We designate the observed spatial density distribution at the known time $t$ by $l_{\ell}^{\prime}(x, y)$.

From the geometry and timing characteristics of the apparatus we have knowledge of the molecular beam distribution at an earlier stage of its evolution. This information is described by a function $h_{y}(x, t)$ which describes the rate of arrival of molecules at a given plane normal to the direction of beam propagation. If we are studying direct beams, i.e., those which have not interacted with any object in their path, the function $h_{y}(x, t)$ is referred to arrival at the plane of the final chopper. In reflected beam studies, this function describes arrival at the target plane. The computation of $h_{y}(x, t)$ is described in section 13.5 and a simplified approximate computation is given in 13.6. In this section, $h_{y}(x, t)$ is considered to be known.

The coordinates in $x-t$ space together with the velocity components $v_{x}, v_{y}$ serve to designate all information of interest about a given molecule. The packet of molecules may be defined by a density function $\rho\left(x, t, v_{x}, v_{y}\right)$, where

$$
d \rho=h(x, t) N\left(v_{x}, v_{y}\right) d x d t d v_{x} d v_{y} .
$$

Molecules leaving the region of $x-t$ space of dimensions $d x$, $d t$ with the exact velocity components $v_{x}, v_{y}$ are found at time $t$ to occupy a parallelogram in $x-y$ space of base $d x$ and altitude $v_{y} d t$. Conversely, the molecules with velocity components $\left(v_{x}, v_{y}\right)$ which are observed in the region of area $d x d y$ must have originated in an area of $x-t$ space equal to

$$
d x d t=\frac{d x d y}{v_{y}}
$$

The total number of molecules observed in the area $d x d y$ is an integral of eq (12.4) with the substitution of expression (12.5) for $d x d t$. Thus we obtain

$$
d \ell_{i}^{\prime}(x, y)=\frac{h(x, t)}{v_{y}} \cdot N\left(v_{x}^{\prime}, v_{y}^{\prime}\right) d v_{x}^{\prime} d v_{y}^{\prime},
$$

where $x$ and $t$ may be determined in terms $x, y$, $v_{x}^{\prime}, v_{y}^{\prime}$ by the equations of motion at constant velocity. It is most convenient in the work which follows to work with a related distribution function

$$
d l\left(v_{x}, v_{y}\right)=t^{2} d l^{\prime}(x, y),
$$

where $v_{x}=x / t, v_{y}=y / t$. The new distribution function is the velocity distribution that we would compute if we assumed that all of the molecules had been at position $x=0, y=0$ at time $t=0$. This approximate starting condition is made as realistic as possible by choosing the origin in 
$x-t$ space at the centroid of the distribution $h(x, t)$. The experimentally determined function $\ell\left(v_{x}, v_{y}\right)$ is thus a first-order approximation to the true velocity distribution function $N\left(v_{x}, v_{y}\right)$. Substituting eq (12.7) into eq (12.6) we find that $\ell\left(v_{x}, v_{y}\right)$ is related to the true distribution by the integral equation:

$$
\ell\left(v_{x}, v_{y}\right)=\iint \frac{t^{2}}{v_{y}^{\prime}} h(x, t) N\left(v_{x}^{\prime}, v_{y}^{\prime}\right) d v_{x}^{\prime} d v_{y}^{\prime} .
$$

Now if we represent the actual molecular velocity components $\left(v_{x}^{\prime}, v_{y}^{\prime}\right)$ as the sum of the apparent velocity components and a correction:

$$
v_{x}^{\prime}=v_{x}+v_{x}^{\prime \prime} ; v_{y}^{\prime}=v_{y}+v_{y}^{\prime \prime}
$$

eq (12.8) may be rewritten as

$\ell\left(v_{x}, v_{y}\right)=\iint \frac{t^{2}}{v_{y}+v_{y}^{\prime \prime}} h(x, t) N\left(v_{x}+v_{x}^{\prime \prime}, v_{y}+v_{y}^{\prime \prime}\right) d v_{x}^{\prime \prime} d v_{y}^{\prime \prime}$.

With the equations of constant velocity motion, i.e.,

$$
\begin{aligned}
& x=v_{x} T=x_{0}+v_{x}^{\prime}(T-t) \\
& y=v_{y} T=y_{0}+v_{y}^{\prime}(T-t),
\end{aligned}
$$

and a bit of algebra, we obtain:

$$
\begin{gathered}
x=T\left(v_{y}^{\prime \prime} \cdot \frac{v_{x}+v_{x}^{\prime \prime}}{v_{y}+v_{y}^{\prime \prime}}-v_{x}^{\prime \prime}\right) \\
t=T \cdot \frac{v_{y}^{\prime \prime}}{v_{y}+v_{y}^{\prime \prime}},
\end{gathered}
$$

and

$$
\begin{array}{r}
\ell\left(v_{x}, v_{y}\right)=\iint f\left(v_{x}, v_{y}, v_{x}^{\prime \prime}, v_{y}^{\prime \prime}\right) N\left(v_{x}+v_{x}^{\prime \prime}, v_{y}+v_{y}^{\prime \prime}\right) \\
d v_{x}^{\prime \prime} d v_{y}^{\prime \prime},
\end{array}
$$

where

$$
\begin{array}{r}
f\left(v_{x}, v_{y}, v_{x}^{\prime \prime}, v_{y}^{\prime \prime}\right)=\frac{t^{2}}{v_{y}+v_{y}^{\prime \prime}} h\left(T\left[v_{y}^{\prime \prime} \frac{v_{x}+v_{x}^{\prime \prime}}{v_{y}+v_{y}^{\prime \prime}}-v_{x}^{\prime \prime}\right],\right. \\
\left.\frac{v_{y}^{\prime \prime} T}{v_{y}+v_{y}^{\prime \prime}}\right) .
\end{array}
$$

Since $f$ is a known function of its four variables, the double integration becomes a linear operator, and in operational notation, eq (12.13) becomes simply

$$
\ell\left(v_{x}, v_{y}\right)=L\left[N\left(v_{x}, v_{y}\right)\right] \text {. }
$$

If $h$ had been a delta function of $(x, t)$, then $\ell$ and $N$ would be identical. If $h(x, t)$ is sharply peaked about the origin, $\ell$ must be a reasonable first approximation to $N$. Thus we write:

$$
N\left(v_{x}, v_{y}\right)=\ell\left(v_{x}, v_{y}\right)+N_{1}\left(v_{x}, v_{y}\right),
$$

and the operational equation becomes

$$
\ell-L \ell=L N_{1} \text {. }
$$

The function $\ell_{1}\left(v_{x}, v_{y}\right)=\ell-L \ell$ may be computed in a straightforward manner. Equation (12.17) above thus becomes

$$
\ell_{1}\left(v_{x}, v_{y}\right)=L N_{1}\left(v_{x}, v_{y}\right) \text {. }
$$

This equation has the same form as the original operational eq (12.15), with a new pair of known and unknown functions. We may thus proceed with additional degrees of approximation by repeating the operation with which $\ell_{1}$ was derived from $\ell$. Thus by induction we arrive at the result

where

$$
N\left(v_{x}, v_{y}\right)=\ell+\sum_{k=0}^{\infty} \ell_{k},
$$

$$
\ell_{k}=\ell_{k-1}-L \ell_{k-1} \text {. }
$$

\subsection{Reliability of the Iteration Process}

The process of successive approximations which has just been derived is an inversion of the double integration process

$$
\begin{aligned}
\ell\left(v_{x}, v_{y}\right)=L \cdot N\left(v_{x}, v_{y}\right) & =\int \mathcal{S} f\left(v_{x}, v_{y}, v_{x}^{\prime \prime}, v_{y}^{\prime \prime}\right) N\left(v_{x}\right. \\
& \left.+v_{x}^{\prime \prime}, v_{y}+v_{y}^{\prime \prime}\right) d v_{x}^{\prime \prime} d y_{y}^{\prime \prime} .
\end{aligned}
$$

The value of $\ell$ at any fixed velocity $\left(v_{x}, v_{y}\right)$ is a weighted in tegral of the value of $N$ in the neighborhood of the given velocity. This averaging process tends to round off any prominent features in $N\left(v_{x_{0}}, v_{y_{0}}\right)$, making $\ell$ an inherently smoother function than $N$. A Fourier transform of $\ell$ is thus relatively lacking in high-frequency components, as compared with the corresponding transform of $N$. The iteration process which we have developeat restores the high-frequency components which were attenuated by the weighted integration.

The way in which this process works may be illustrated by applying our iteration method in a thought experiment to the inversion of a convolution integral in one dimension. The resulting conchusions must be applied with caution to our problem, since the double integration process $L \cdot N\left(v_{x}, v_{y}\right)$ is not exactly a convolution process. It differs from a convolution process in that the multiplier $f$ is a function of the fixed variables $v_{x}, v_{y}$ as well as of the dummy variables $v_{x}^{\prime}, v_{y}^{\prime}$. Thus, we limit the use of convolution theorems to prediction of experimental error, rather than attempting to use convolution to find $N\left(v_{x}, v_{y}\right)$. In our experiment $N(y)$ is an unknown function to be determined by experiment and calculation. The apparatus is designed to yield the output function

$$
\ell(x)=L \cdot N(x)=\int_{-\infty}^{\infty} f(y) \cdot N(x+y) d y,
$$

where $f(y)$ is a known normalized function 


$$
\left(\int_{-\infty}^{\infty} f(y) d y=1\right)
$$

Because of various experimental errors, the apparatus yields an output function

$$
\ell_{m}(x)=\ell(x)+\ell_{e}(x),
$$

where $\ell_{e}(x)$ represents experimental error. If we succeed in exactly inverting the integral equation, we obtain an experimentally measured value of $N$, which differs from the true value by an error function

$$
N_{m}(x)=N(x)+N_{e}(x) .
$$

By definition, $N_{m}(x)$ must satisf y the relationship

$$
\ell_{m}(x)=L \cdot N_{m}(x),
$$

and hence, by subtraction

$$
\ell_{e}(x)=L \cdot N_{e}(x) .
$$

If we take the Fourier transforms of both sides of these equations, i.e., $\bar{g}(\omega)=\frac{1}{\sqrt{2 \pi}} \int_{-\infty}^{\infty} g(x) e^{-i \omega x} d x$, the convolution theorem [47] provides the relationships:

$$
\begin{aligned}
\bar{\ell}_{m}(\omega) & =\bar{f}(\omega) \cdot \bar{N}_{m}(\omega) \\
\bar{\ell}(\omega) & =\bar{f}(\omega) \cdot \bar{N}(\omega) \\
\bar{\ell}_{e}(\omega) & =\bar{f}(\omega) \cdot \bar{N}_{e}(\omega)
\end{aligned}
$$

where the bar over a function signifies the Fourier transform of the corresponding function of $x$. The apparatus thus reacts to the unknown input function with a frequency response $\bar{f}(\omega)$. Since the integral $\bar{l}(\omega)$ representing the convolution is a weighted average of values of $N(x)$ at neighboring values of $x$, we can expect precise reproduction of slowly varying functions of $x$ and extreme attenuation of rapid fluctuations. Thus $\bar{f}(\omega)$ approaches unity at low values of $\omega$ and approaches zero at high values of $\omega$. The transforms of $N(x), N_{m}(x)$, and $N_{e}(x)$ may be obtained by inverting the above equations:

$$
\begin{aligned}
& \bar{N}_{m}(\omega)=\frac{\bar{l}_{m}(\omega)}{\bar{f}(\omega)} \\
& \bar{N}(\omega)=\frac{\bar{l}(\omega)}{\bar{f}(\omega)} \\
& \bar{N}_{e}(\omega)=\frac{\bar{l}_{e}(\omega)}{\bar{f}(\omega)} .
\end{aligned}
$$

The fact that $\bar{f}(\omega)$ approaches zero at large values of $\omega$ provides no special difficulty, since $\bar{l}(\omega)$ has been forced to be correspondingly small by the response of the instrument. The measurement error $\ell_{e}(x)$ is not necessarily so deficient in high-frequency components, however, and it is entirely possible that $\bar{N}_{e}(\omega)$ may increase indefinitely as $\omega$ approaches infinity. In fact, the random character of grain noise on photographic emulsions suggests that $\bar{\ell}_{e}(\omega)$ should approach a constant value as $\omega$ approaches infinity. A complete correction for the smoothing effect of the finite instrumental frequency response is thus rendered impossible by the presence of high-frequency noise in the measured response function.

It is instructive, now, to observe the frequency responses of the successive approximations obtained by applying our iteration process to the conversion of experimental data. The iteration process yields the series of functions of $x: \ell_{m, 0}(x), \ell_{m, 1}(x), \ell_{m, 2}(x) \ldots$ satisfying the relations

$$
\begin{gathered}
\ell_{m, k}(x)=\ell_{m,(k-1)}(x)-L \ell_{m,(k-1)}(x) \\
\ell_{m, 0}(x)=L \cdot N_{m}(x) .
\end{gathered}
$$

Successive approximations to $N_{m}(x)$ are $N_{m, k}(x)$ $=\sum_{j=0}^{k} l_{m, k}(x)$.

Applying the Fourier transform to these condition yields

$$
\bar{\ell}_{m, k}(\omega)=\bar{l}_{m,(k-1)}(\omega)[1-\bar{f}(\omega)] .
$$

Hence, by induction

$$
\bar{l}_{m, k}(\omega)=\bar{l}_{m, 0}(\omega)[1-\bar{f}(\omega)]^{k}
$$

and

$$
\begin{aligned}
\bar{N}_{m, k}(\omega)= & \bar{\ell}_{m_{t} 0}(\omega) \cdot \sum_{j=0}^{k}[1-\bar{f}(\omega)]^{j} \\
& =\bar{N}_{m}(\omega) \cdot \bar{f}(\omega) \sum_{j=0}^{k}[1-\bar{f}(\omega)]^{j} .
\end{aligned}
$$

Now if we let $\bar{g}(\omega)=1-\bar{f}(\omega)$, the equation becomes

$$
\bar{N}_{m, k}(\omega)=\bar{N}_{m}(\omega)[1-\bar{g}(\omega)] \sum_{j=0}^{k} \bar{g}(\omega)^{j}
$$

or

$$
\bar{N}_{m, k}(\omega)=\bar{N}_{m}(\omega)\left[1-\bar{g}^{k+1}(\omega)\right] .
$$

Now $g(\omega)$ is close to zero at low values of $\omega$ and approaches unity from below as $\omega$ approaches infinity. The behavior of $\bar{g}^{k}(\omega)$ for several values of $k$ is illustrated in figure $19 \mathrm{a}$.

The result of a finite number of iterations is related to the limiting result of an infinite number of iterations by the transform equation

where

$$
\bar{N}_{m, k}(\omega)=\bar{f}_{k}(\omega) \bar{N}_{m, \infty}(\omega),
$$

$$
\bar{f}_{k}(\omega)=1-\bar{q}^{k+1}(\omega) .
$$

The effect of a few iterations is thus to raise the 
frequency response nearly to unity in the range of frequencies where an appreciable response was originally present, while providing only a small increase of response in regions where the original response was low. This is illustrated in figure $19 \mathrm{~b}$. In the limit as $k$ approaches infinity, the effective frequency response approaches unity at all frequencies.

If the characteristics of the unknown function $N(x)$ are well enough known in advance to yield the general shape of $\bar{N}(\omega)$ and hence of $\bar{l}(\omega)$, and the noise spectrum $\bar{l}_{e}(\omega)$ is known, we can use this information to choose $k$ for optimum conversion of the data. The ideal data function $\bar{l}(\omega)$ can be expected to be larger than $\bar{l}_{e}(\omega)$ for frequencies below a certain value $\omega_{c}$ and to be smaller than $\bar{l}_{e}(\omega)$ for higher frequencies. The response to any frequency below $\omega_{c}$ thus yields more signal than noise and vice versa. The maximum fidelity to meaningful signal consistent with adequate noise rejection will occur if the steepest slope in $\bar{f}_{k}(\omega)$ occurs in the neighborhood of $\omega_{c}$. The transition from nearly unity response to nearly zero response thus occurs in the neighborhood of the lowest frequency at which noise exceeds signal.

In practical use of the iteration, one would not attempt to match $f_{k}(\omega)$ to estimated signal and noise spectra. Instead, one would simply carry out successive iterations and pick out the most convincing solution on the basis of expected properties of the unknown function, just as a microscopist carries out through-focus studies and selects the most convincing picture. The above thought experiment serves, however, to indicate strongly that the result of such an iteration process is likely to extract close to the maximum of useful information from the experimental data.

In this section, we have outlined the principles to be employed in analysis of the data, where a complete velocity distribution is desired. In many cases, the desired information may be less detailed and considerable simplification of the procedure may be feasible. Such simplifications are most efficiently made by the person wishing to obtain the velocity information, and were, therefore, considered beyond the scope of this project.
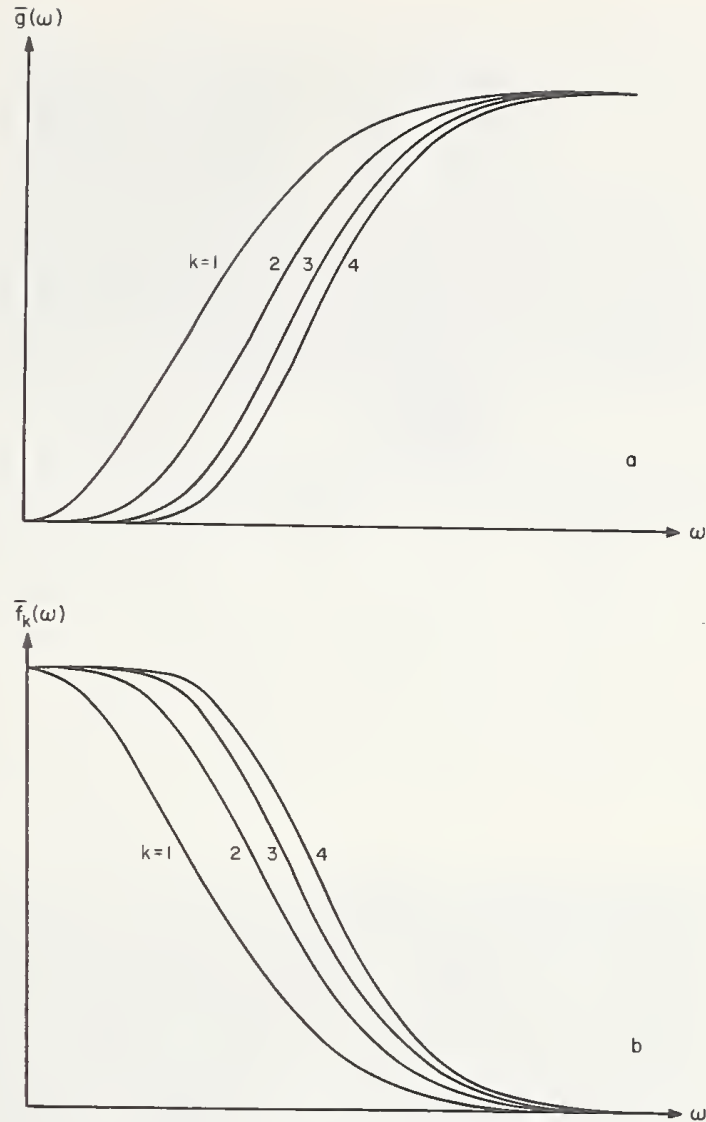

FIGURE 19a. Variation of the function $\mathrm{g}^{\mathrm{k}}(\omega)$ for successive values of $\mathrm{k}$.

In this illustrative example, $g_{(\omega)}$ was chosen as $1-e^{-\omega^{2}}$. The function $g^{\prime}(\omega)$ may be regarded as a measure of frequency distortion in the result of the kth iteration in the process of computing $N(x)$.

FIGURE 19b. Variation of the frequency response function $\mathrm{f}_{\mathrm{k}}(\omega)$ after several successive iterations.

As the iteration process proceeds, the frequency response is made to approach unity in regions where it was initially high, while considerably smaller gains occur in regions where the initial response was poor. The initial response function $f_{1}(\omega)$ was chosen as $e^{-\omega^{2}}$ in consistency with figure $\alpha$. Figures $19 \mathrm{a}$ and $19 \mathrm{~b}$ are mirror inversions of each other. From this figure we see that the process of successive approximations produces a region of excellent response in the low frequency region and gradually extends the high response region to higher frequencies. The response to the relatively low frequency signal spectrum is thus improved far more than the response to high frequency noise is increased.

\section{Appendix}

\subsection{The Photographic Camera}

There are several methods with which one can detect electron beams. When the desired information is in the form of an image, e.g., electron microscope images, the simplest and most reliable method is to use photographic plates. The plates provide a compact permanent record, which may b́e analyzed in whatever detail seems initially suitable without jeopardizing the possi- bility of more detailed analysis if desired at a later time. Since our recording problem is quite similar to that in the electron microscope, we decided to use photographic plates to record the electron optical images.

The technique of using photographic plates in electron optical work differs in only two ways from that of the light optical analogue. First, the electrons can have no obstruction in their path to the plate; second, the exposure must be made under hard vacuum condition lest the electron 


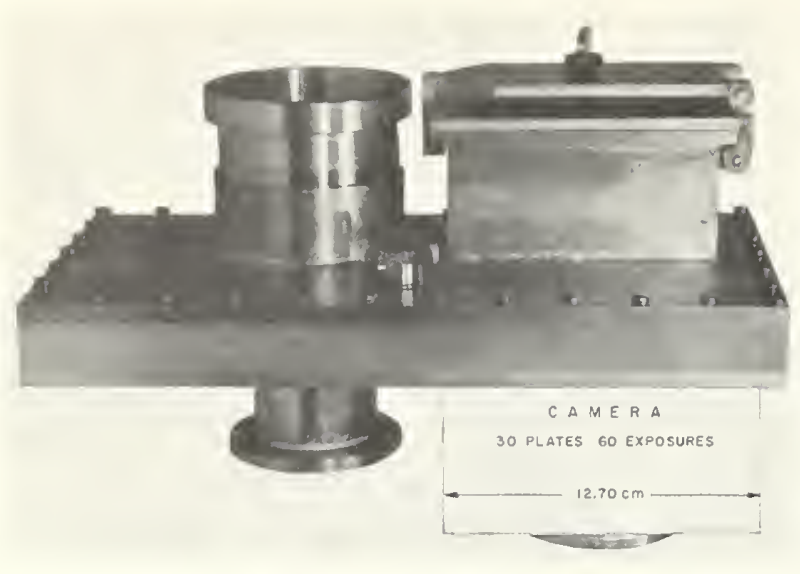

Figure 20. Top view of camera exterior.

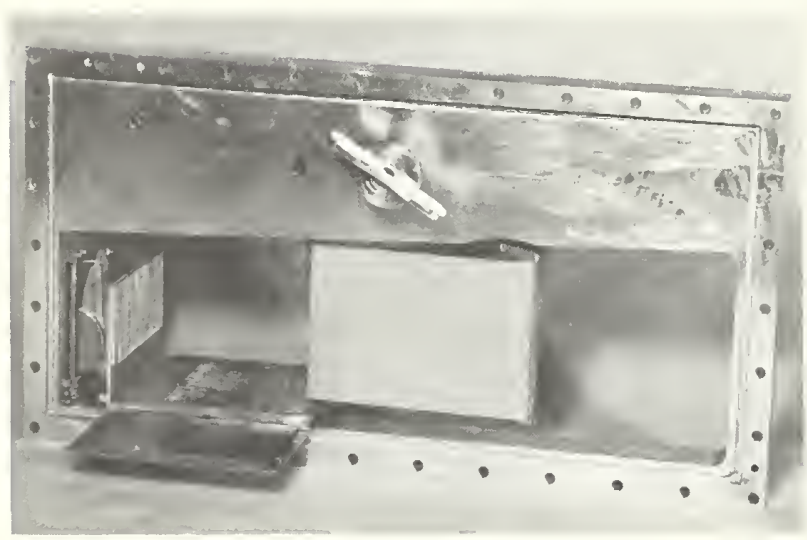

Figure 21. Center section of camera as seen from side facing the operator.

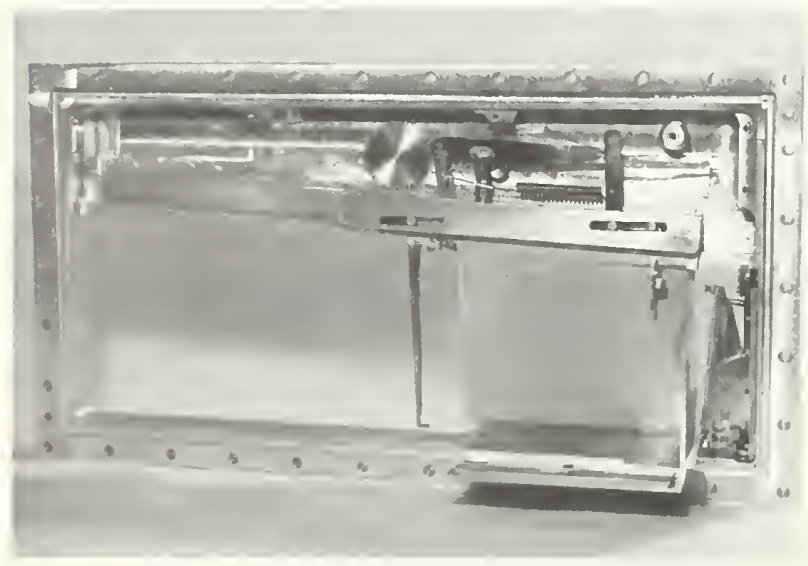

Figure 22. Center section of camera as seen from side facing beam source. -image become diffused by scattering from the residual gas molecules. The processing of the plates is identical to that used in the light optical analogue.

The requirements on the camera were such that it was necessary to design and build this unit in our own laboratory. In addition to storing and manipulating the sensitized plates, the camera had to be capable of being evacuated to a pressure of $10^{-6}$ Torr or better. Also, to enable the operator to see and control the image produced by the apparatus, a phosphorescent viewing screen had to be built into the device in such a way that the screen could be replaced with a photographic plate when an exposure was to be made. In addition, it is very desirable to provide for removing the exposed plates and reloading the camera without disturbing the vacuum in the main chamber. The camera described below fulfills all these requirements.

The construction materials used were all nonmagnetic; namely, brass, aluminum alloy, phosphor bronze, stainless steel type 304, glass, and an epoxy resin to avoid interfering with the magnetic fields used to control the optics of the electron beam.

The camera consists of three main parts bolted together, as can be seen in figure 20. The center section, as seen in figures 21 and 22 , contains the mechanisms for manipulating and storing the plates. By studying figures 21 and 22 one can see that by turning the knurled knob, one causes the parallel clamp or tongs to pick up a plate from the magazine and carry it to the area immediately in front of the viewing screen where the exposure may be made. When the motion is reversed, the plate is deposited in the magazine. If the reverse motion is continued, overriding the stop, the magazine is advanced to the next position, bringing the next plate in line for the next exposure. The advance mechanism employs a simple ratchet system where the short tong, as seen in figure 22 , presses against two lever arms. One arm unlocks the magazine while the other causes

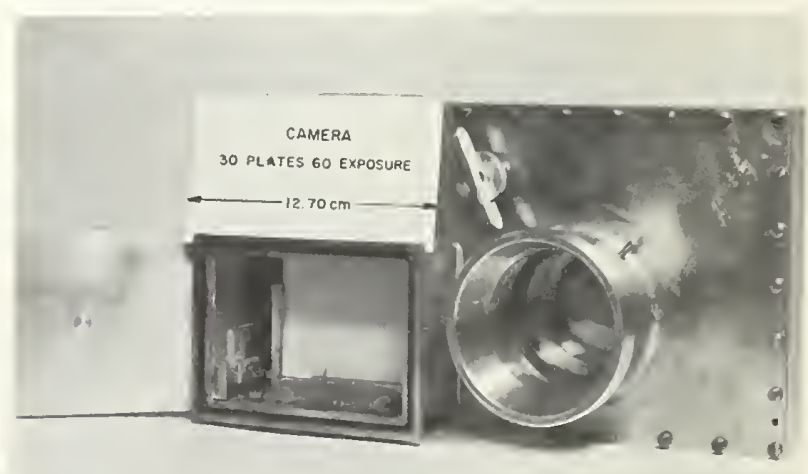

Figure 23. External view of camera as seen by operator. 
the magazine to advance one notch. On reverse motion of the tong, a spring, seen in figure 22 , forces the alining wedge to engage and lock the magazine in place. Simultaneously the ratchet cam advances to the next groove, leaving the magazine in line and locked in position for the next cycle.

On the front section, as seen in figures 20 and 23 , are the viewing screen-vacuum seal device and the port through which the film magazine is loaded into the camera. The vacuum seal on this port opening is simple and self-explanatory. The screen-vacuum seal port is simply a metal tube in which a thick glass disk was sealed in place near one end with epoxy resin. On the end of the tube a recess was made to hold an O-ring which seals off the camera chamber when the tube is advanced into contact with the back plate of the camera. To make the vacuum seal between the cylinder wall and that of the camera, an O-ring groove was machined in the front plate to form a "Wilson seal," thus allowing the cylinder to move along its axis and rotate. With the diameter of the viewing port being about 3 in. and with the camera pumped to a high vacuum, the pressure on this surface was slightly over a hundred pounds. This, of course, makes it difficult to pull open the valve. Although it is not shown in the photograph, the solution to this problem simply consisted of adding a fine threaded section to the inner cylinder and attaching a "nut" to the outer one. Thus, by rotating the cylinder, one could force the inner cylinder to move along its axis.

The back section consists of a flat plate with two ports. One port is connected to a vacuum pump for prepumping the camera chamber before it is opened to the main section of the apparatus. The other is in line with the viewing screen valve located on the front plate. It is attached to the accelerator by a conventional O-ring groove flange. This camera proved to be reliable and was used throughout the work.

\subsection{Earth's Field Neutralization}

The most direct way to eliminate the misalinement caused by the action of the earth's magnetic field is to produce an equal and opposite field. A field of sufficient strength is readily produced by a configuration of wire carrying an electric current. In general, such a field is non-uniform and thus provides only partial compensation which is restricted to a limited region in space.

It is possible, however, to enclose certain spaces with a continuous distribution of circulating current such that the induced magnetic field within the space is uniform. For example, a sphere may have surface currents in circular paths about its $z$-axis with surface current density proportional to $\cos \theta$, where $\theta$ is the angle between the axis and a radius to any point on the sphere. Similarly, an infinitely long cylinder may have surface currents in longitudinal paths with current density proportional to $\cos \theta$, where $\theta$ is again measured between the field direction and a radius to the point in question. These continuous current distributions may be effectively simulated by configurations consisting of a finite number of conductors. The resulting fields are quite smooth near the center of the enclosed space, but depart progressively from the uniform central field as the boundaries are approached. It is thus necessary for the entire region traversed by the electrons to be within a sphere or cylinder of much smaller diameter than that of the surface containing the conductors if a high degree of field compensation is to be obtained.

If the spherical geometry were to be used, this requirement would mean that the diameters of the compensating coils would have to be large in comparison with the length of the optical path from the source to the image plane. The impracticality of such an arrangement requires no elaboration. With cylindrical geometry, the variation in directions transverse to the optic axis will be small, as long as the radius of the cylinder carrying the conductors is several times greater than the largest distance from the axis permitted for an electron.

In mounting conductors outside the walls of the vacuum system, this requirement is almost automatically fulfilled. Field variations as a function of the $z$ coordinate are more difficult to control, but even here the cylindrical geometry presents less difficulty than the spherical case. The conductors parallel to the axis must be terminated at some reasonable distance beyond the limits of the optical paths, and connecting wires across the ends of the imaginary cylinder complete the current loops. For good field uniformity along the $z$-axis, the extension of the conductors beyond the optical limits should be large in comparison with the cylinder radius. If this requirement is carried out, the observer watching the fluorescent screen must sit inside the current loops. We shall show presently, however, that field compensation within 5 percent of the earth's field can be obtained without accepting such awkwardly long coils.

In general, a set of coils compensates for only one component of the earth's field. Since the experimentalist generally likes to choose the orientation of his apparatus in accordance with mechanical convenience, it is to be expected that both horizontal and vertical transverse field components will be present if the optical axis is horizontal. It is possible for a single set of coils to balance out the two transverse field components if it is properly oriented by rotation about the optic axis. It is more convenient, however, to adjust for precise compensation by varying the currents in two sets of coils rotated at right angles to each other. Thus, the mechanical supports of the coils can be fixed and the adjustments may be made by setting a potentiometer.

The above arrangement makes no provision for balancing out the axial component of the earth's field. Instead of deflecting the electron paths as the other two components do, this field acts like 
a continuous distribution of weak magnetic lenses. The focal power of this distributed lens becomes important in distances of the order of

$$
\frac{1}{\sigma_{H}}=2 c \frac{\sqrt{\frac{2 m}{e} \cdot \phi}}{H}=\frac{6.75 \mathrm{~V}^{1 / 2}}{H} \mathrm{~cm}
$$

where $V$ is the accelerating potential of the electron beam in volts and $H$ is the earth's magnetic field in oersteds. If we attempted to work with electron beams with only a few volts of accelerating potential, this focusing effect would become fairly important; but a variety of annoyances, which are easy to accept when working with beams at higher potentials, would complicate operations much more seriously than this focusing effect. Consequently, the electron beam is never operated at voltages low enough to provide serious focusing effects from this source; and there is no practical need to cancel out the axial component of the earth's field.

Returning to the problem of cancelling out the transverse components of the earth's field, there remains the question of the number of compensating coils to be arranged about the imaginary cylinder. Neglecting end effects, each long rectangular coil may be thought of as a pair of wires carrying currents in opposite directions parallel to the axis. Imagining the coils to be in horizontal planes (thus providing a vertical compensating
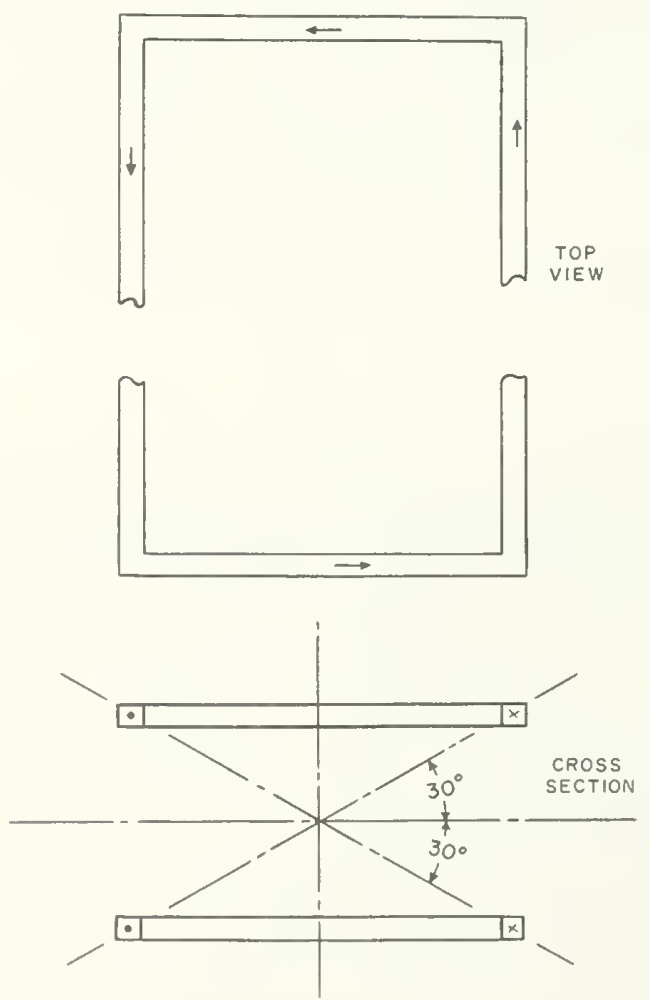

FIgURE 24. Top view and cross-sectional view of optimum pair of elongated rectangular coils for field compensation. field component), conditions of symmetry require that each coil above the $x$ axis should be matched with a duplicate coil at the same distance below the axis. "The induced vertical field on the $y$ axis is then an even function of $y$.

If $H(y)$ is expanded as a Taylor series, the result is $H(y)=H_{0}+H_{2} y^{2}+H_{4} y^{4}+\ldots$. If only one compensating coil is used, the first unwanted term will be $\mathrm{H}_{2} y^{2}$. If two coils are used, we can arrange the coil positions such that $H,=0$ and the first unwanted term is $H_{4} y^{4}$. Similarly, as additional coils are used, one more term can be eliminated from the series for each additional coil. Unless accurate compensation is needed over a wide range of $y$ values, a very small number of coils proves to be adequate. In the present experiment, two coils were used for each transverse field component. The use of two coils provides more accurate field compensation than one, but the more obvious reason for not using a single coil is the fact that one coi! in the horizontal plane would get in the way of an observer looking at the fluorescent screen. Thus, the practical choice is whether to use two or four coils to neutralize each field component. In the two-coil arrangement, the wires and current directions are arranged as shown in figure 24 .

The vertical component of the magnetic field due to one of the wires is given by $H_{n}=\frac{2 i}{r_{n}} \cos \theta_{n}$, where $r_{n}$ is the distance from the point in question to the wire, $\theta_{n}$ is the slope angle of the line connecting the point to the wire, and $i$ is the current in abamperes or one-tenth the current in amperes. Thus, if the vertical distance between the coils is $2 d$, the vertical field strength along the axis becomes

$$
\begin{aligned}
H(y) & =\frac{4 I d \sqrt{3}}{10}\left[\frac{1}{4 d^{2}+y^{2}+2 d z}+\frac{1}{4 d^{2}+y^{2}-2 d z}\right] \\
& =\frac{0.8 \sqrt{3}}{d\left(4+\frac{\sigma^{4}}{4+\sigma^{2}}\right)}
\end{aligned}
$$

where $\sigma=\frac{y}{d}$, and $I$ is the current in amperes.

The variation of field strength along the $y$ axis is then approximately

$$
\frac{\Delta H}{H}=-\left(\frac{\sigma}{2}\right)^{4}
$$

thus providing field compensation within 0.4 percent for values of $|y / d|$ up to 0.5 . Within the range $|r|<d / 2$ we are assured that the field is comparably uniform. This can be seen when the scalar potential is written as a series of spherical harmonics:

$$
\psi(z)=\frac{0.2 \sqrt{3} I}{d}\left[r \cos \theta-\frac{1}{80 d^{4}} r^{5} P_{5}(\cos \theta)+\ldots\right]
$$




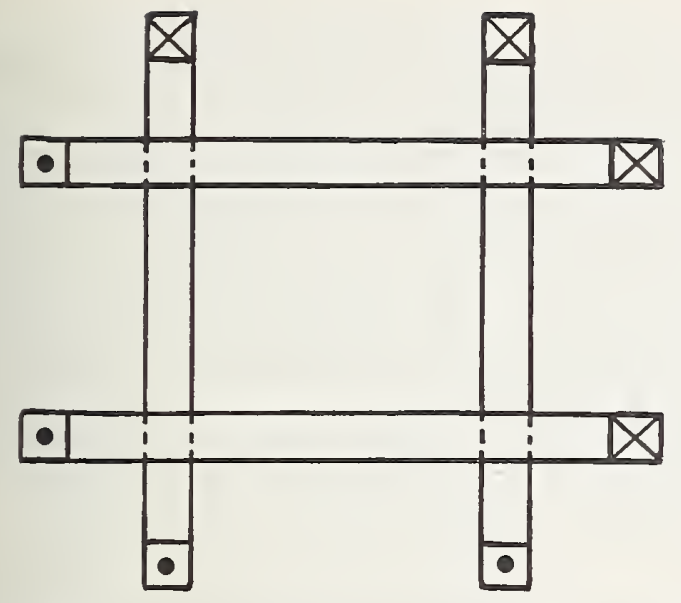

Figure 25. Pair of Helmholtz coil sels for compensating both transverse components of the earth's magnetic field.

With conventional currents in directions indicated, horizontal colls produce a field directed upward and coils in vertical plane produce a field directed to the left. In practice, current directions are readily checked with a hand compass.

where $r$ and $\theta$ are polar coordinates, and the $P_{n}(\cos \theta)$ are Legendre polynomials. Thus, the line integral of $\Delta H / H$ from the plane $y=0$ to any point on the sphere of radius $d / 2$ does not exceed

$$
\frac{\Delta \psi}{H_{0}} \leqq \frac{d}{80 \cdot 2^{4}}=\frac{d}{1280} .
$$

When two sets of coils are used to compensate for both the horizontal and the vertical component of the earth's field, the configuration of wires becomes that shown in figure 25 . In the present experiment, however, it was felt that the inconvenience of having coils run almost directly above and below the optical system was not justifiable. In most of the United States, the earth's magnetic field is inclined at an angle of $60^{\circ}$ or more to the horizontal. The horizontal component of the earth's field is thus roughly half of the vertical component, and the portion of that component which is transverse to the optic axis is somewhat smaller, depending upon the orientation of the instrument. Thus, the horizontal field to be neutralized is smaller than the vertical field, and the required degree of compensation is correspondingly less vigorous. It is convenient to build the compensating coils in the form of a box, as shown in figure 26.

The horizontal compensating field is given approximately by

$$
H(x)=\frac{0.8 I}{d\left[1+\frac{\sigma^{2}}{4}-\frac{3 \sigma^{2}}{1+\frac{\sigma^{2}}{4}}\right]}
$$

where $\sigma=x / d$. The presence of terms proportional to $\sigma^{2}$ in the denominator limits the range within which field compensation is as good as at the end

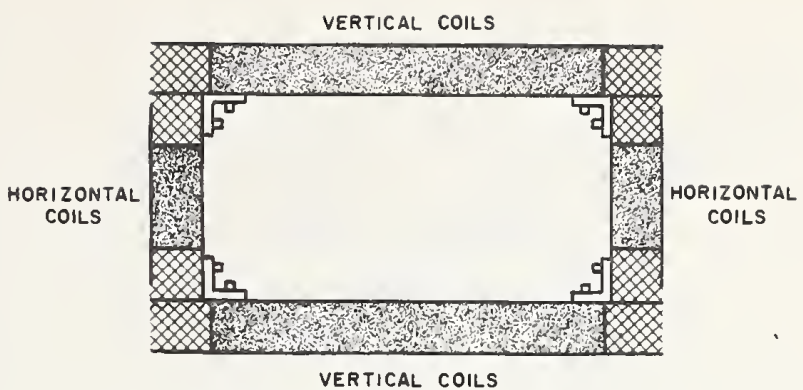

Figure 26. Practical configuration of compensating coils as used in this apparatus.

points for the vertical field to $|x / d|<0.0376$, as compared with $|y / d|<0.5$. The difference between these two ranges illustrates strikingly the importance of adhering to the geometry which gives maximum field compensation.

The above calculations were based on the assumption of infinitely thin single conductors. Actual coils consist of a large number of turns of wire in series, and the total cross section of wire, insulation, and interstices between wires may amount to several square centimeters. It is thus proper to raise a question as to the validity of the approximations made in the calculations. The presence of $n$ conductors instead of one merely requires that the coil current should be $I_{\text {coil }}=I / n$.

The replacement of a filamentary current with a distributed current causes some modification in the field. It is worth noting, however, that a uniform current distribution in a circular conductor of any size produces the same external magnetic field outside the conductor as is produced by a filamentary current. Thus, if we draw a circle of equal cross-sectional area about the current-carrying cross section, the deviation from results for a filamentary conductor may be thought of as the resultant of equal and opposite currents in the areas not common to both the circle and the actual current-carrying cross section.

If the largest dimension of the current-carrying cross section is $\omega$, and the fraction of cross section not in the circle is $\eta$, it is easy to show that an upper limit for the relative deviation of the field at the center of the Helmholtz coil structure from the calculated value is $\eta \omega^{2} / 16 d^{2}$, a number which is less than 0.01 for all practical values of $\omega / d$.

\subsection{Variation of the Compensating Field With Position Along the $z$-Axis}

In order to compute the end effects for a pair of Helmholtz coils of finite length, we use the formula for the vertical component of field at any point due to current in a straight wire of finite length

$$
H_{y}=\frac{i}{r}\left(\sin \theta_{1}+\sin \theta_{2}\right) \cos \phi,
$$




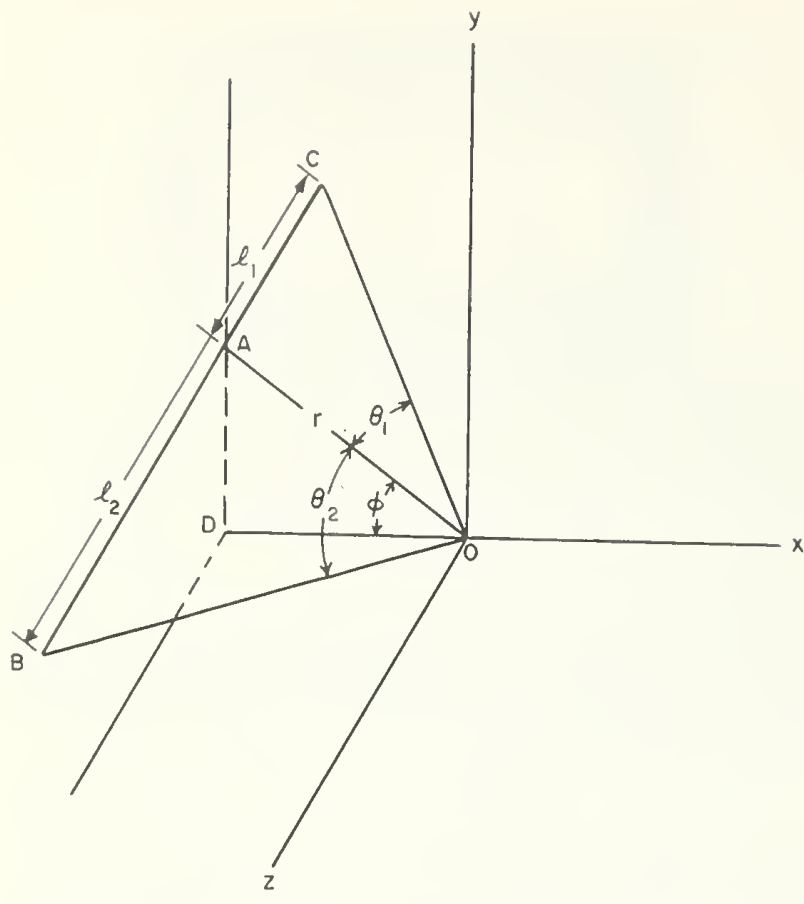

FIGURE 27. Diagram giving nomenclature for compensating field calculations.

The wire segment $\mathrm{BC}$ is horizontal, and the plane $\mathrm{OAD}$ is normal to the wire. The point $O$ is the point at which the vertical field component is to be computed.

where $r, \theta_{1}, \theta_{2}$ are shown in figure 27 .

The plane $O A D$ is drawn perpendicular to the straight wire $B C$ and through the point of interest $O$. The line $O D$ is horizontal. By simple geometry, $\sin \theta_{1}=\frac{\ell_{1}}{\sqrt{r^{2}+\ell_{1}^{2}}}$, and $\sin \theta_{2}=\frac{\ell_{2}}{\sqrt{r^{2}+\ell_{1}^{2}}}$. In the case of longitudinal wires, $l_{1}$ and $l_{2}$ are distances from the point in question to opposite ends of the coil, $r$ is equal to $2 d$, and $\phi$ is always $30^{\circ}$ for the coils producing a vertical field.

The electrical circuit is completed at each end of each coil by transverse wires for which $\ell_{1}=l_{2}$ $=d \sqrt{3}, r=\sqrt{\Delta z^{2}+d^{2}}$, and $\cos \phi=\frac{\Delta z}{\sqrt{\Delta z^{2}+d^{2}}}$, where $z$ is the distance to the end of the coil. If distances to opposite ends of the coil are written as $\Delta z_{1}, \Delta z_{2}$, and for convenience $\sigma_{1}=\frac{\Delta z_{1}}{d}, \sigma_{2}=\frac{\Delta z_{2}}{d}$, the total field on the axis at any point becomes

$$
H_{\nu}=\frac{i \sqrt{ } 3}{d}\left[f\left(\sigma_{1}\right)+f\left(\sigma_{2}\right)\right],
$$

where

$$
f\left(\sigma_{j}\right)=\frac{\sigma_{j}}{\sqrt{\sigma_{j}^{2}+4}}\left[1+\frac{4}{\sigma_{j}^{2}+1}\right]
$$

The function $f\left(\sigma_{j}\right)$ approaches unity as $\sigma_{j}$ approaches infinity. As $\sigma_{j}$ is progressively lowered, the function rises to a maximum value of 1.359 at $\sigma_{j}=1.207$, and then falls to zero at $\sigma_{f}=0$. At $\sigma_{j}=5$, however, the value of $f$ is only 1.07. In a long coil with $\sigma_{1}+\sigma_{2} \gg 1$, the term in which $\sigma_{j}$ is large is very close to 1 , and the fractional deviation of the field from the asymptotic value is thus just half of the difference between unity and $f\left(\sigma_{j}\right)$ for the small value of $\sigma$. We can thus maintain a neutralizing field of uniform strength within 18 percent for all points up to positions closer to the end of the coil than $1.2 d$ and within 3.5 percent for positions more than $5 d$ distant from the ends of the coil.

For the sake of completeness, we now give the expression for $H_{x}$ when the same geometry is used to generate a horizontal field:

$$
H_{x}=\frac{1}{d}\left[g\left(\sigma_{1}\right)+g\left(\sigma_{2}\right)\right],
$$

where

$$
g\left(\sigma_{j}\right)=\frac{\sigma_{j}}{\sqrt{\sigma_{j}^{2}+4}}\left[1+\frac{4}{\sigma_{j}^{2}+3}\right] .
$$

Again, the difference between the actual field and the field of infinitely long coils is small wherever $\sigma_{1}$ and $\sigma_{2}$ are greater than 5. Since values of $\sigma$ this large are needed for good field compensation and the excess length of coil needed is thus $5 d$, it is a good general rule that the lateral dimension of the compensating coil should be as small as practicable for clearance around the vacuum walls of the system.

\subsection{Relative Positions of the Chopper Disks}

The mean velocity of the molecular beam packet selected by the chopper disks is determined by the distance between the planes of the disks and the delay between the times at which the two slits are centered with respect to the molecular beam. More precisely, a range of velocities is transmitted, as determined by the slopes of lines drawn between slit jaws in figure $31 \mathrm{~b}$. The widths of the two sets of chopper slits thus enter into the range of velocities transmitted.

Since it is easier to vary the time delay between the two chopper disks than to cut new slits on a chopper disk, it is worthwhile to plot the velocity ranges that will be transmitted for different relative displacements of the disks. This is done for a particular set of slit widths in figure 28 .

We can pick the range of velocities corresponding to any relative displacement on the table by displacing the second chopper disk by a suitable angle with respect to the first in the direction opposite to the rotation of the pair. The required displacement angle is given by $\theta_{d}=d / r_{M}$, where $d$ is the displacement distance and $\dot{r}_{M}$ is the distance from the rotation axis to the middle of the slit. We designed the chopper disks with a simple locating mechanism for setting the disks at any desired orientation relative to each other.

The second chopper disk fitted onto a hub which was an integral part of the first disk as shown in 


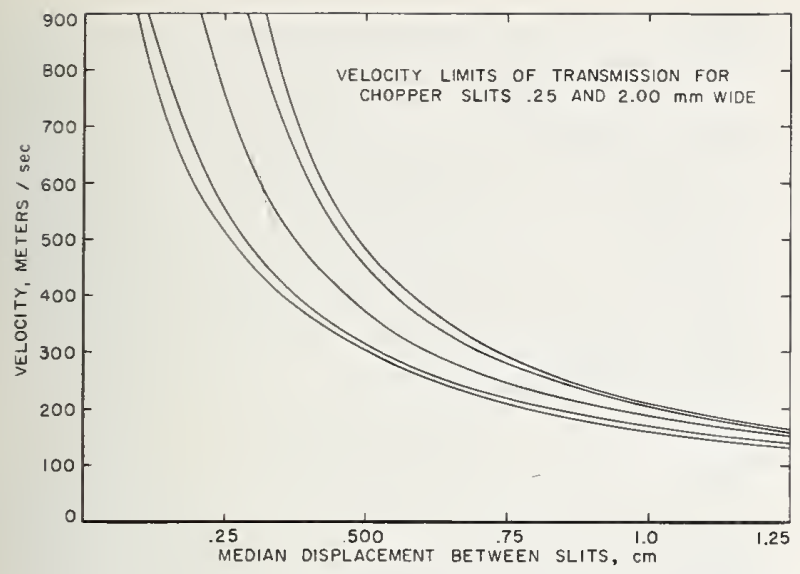

FIGURE 28. Nominal and limiting transmission velocities for slits as used in velocity selector.

Middle curve is nominal velocity based on $8.9 \mathrm{~cm}$ mean chopper radius, $8000 \mathrm{rpm}$ rotational velocity, and $2.5 \mathrm{~cm}$ distance between chopper planes. Neighboring curves give range of relocities within which maximum transmission will occur with slit widths of $2.0 \mathrm{~mm}$ and $0.25 \mathrm{~mm}$. Outer curves give velocity limits outside of which no beam transmission occurs. Taper from maximum transmission to zero transmission is approximately linear.

figure 29. On circles of appropriate diameter on each disk, holes were drilled normal to the disk plane. Dowel pins passing through a solid washer, into which appropriate mating holes were drilled, secured the two disks in a fixed orientation with respect to each other. Two pins at diametrically opposite positions were required to secure each disk to the washer. The angular orientation of the two disks with respect to each other thus depended upon the angular positions of the alining holes in each of the disks and on the two corresponding circles on the washer. By drilling a number of holes at selected angular spacings on both disks and on the corresponding hole circles of the washer, we can greatly increase the number of orientations available. The angular spacings of the holes in each set are shown in figure 30.

The holes are unevenly spaced in each hole circle except the inner circle of the guide ring. The separate possible orientations of the disks with respect to the guide ring are so chosen that 600 different combinations are possible, giving 300 different relative positions of the chopper disks with respect to each other. Thus, the range of relative orientations from $-30^{\circ}$ to $+30^{\circ}$ is covered in $0.2^{\circ}$ intervals. The method for finding the correct hole combination for a given displacement is given below.

The holes are numbered in each circle for convenience in determining proper settings. On the guide ring a double scribe line runs through a set of four holes. The centers of these four holes lie on a common line through the center of the ring. A letter $L$ determines the left side of the ring in the preferred orientation. With the row of holes horizontal and the $L$ on the left, we number the hole pairs $1,2,3,4,5$, starting with the pair on the right within the lines and proceeding in the counterclockwise direction. Likewise, on the disks

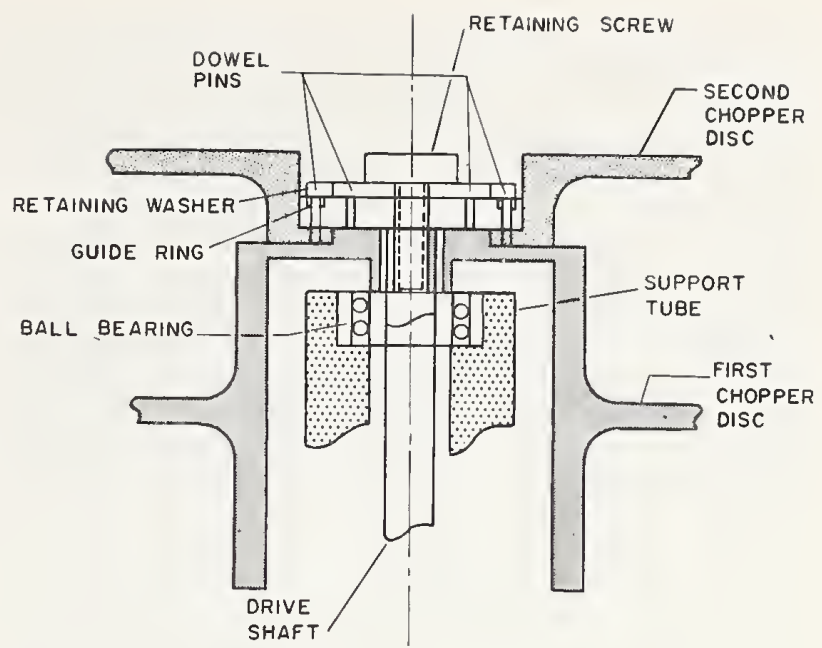

FIGURE 29. Cross-sectional view of the chopper disks and their connection to the driving shaft.

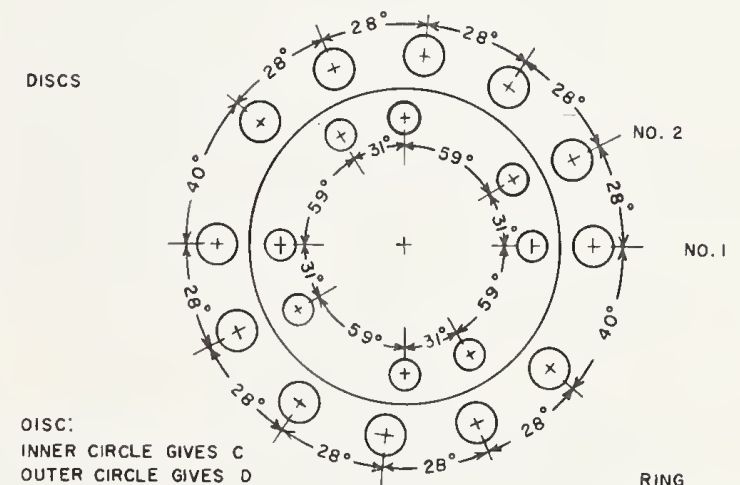

OUTER CIRCLE GIVES $D$ RING

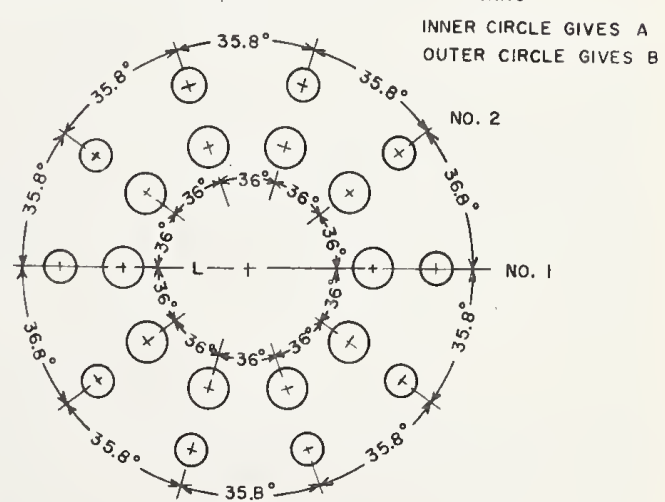

FIGURE 30. Angular spacing of holes in the chopper disks and in the guide rings for provision of known angular spacings between the two chopper disks.

we number the holes consecutively in the counterclockwise direction from a recognizable number one hole. In each disk the number one hole is precisely in line with a slit. When the number one hole is facing to the right, the hole-to-hole spacing just below it is noticeably larger than the corresponding spacing above it. Thus, the interval $1-2$ is always smaller than the interval 11-12. The above information should be sufficient 
to locate the number one hole on each disk and to determine the number associated with each hole. If any difficulty is encountered, this same information, along with the actual spacings of holes in degrees, is given in the drawing.

The chopper rotates in the counterclockwise direction, as seen from the target, and we want the clisk nearest to the source to lead the other one. In the following table, the lead is given in degrees for various hole combinations. A positive number indicates lead in the sense given above, while a negative number indicates lag. The number of the desired holes in each circle is given by an appropriate letter.

Displacement table (degrees advance in counterclockwise rotation)

$a b c$ $d$ $\begin{array}{llll}a & b & c\end{array}$ $d$

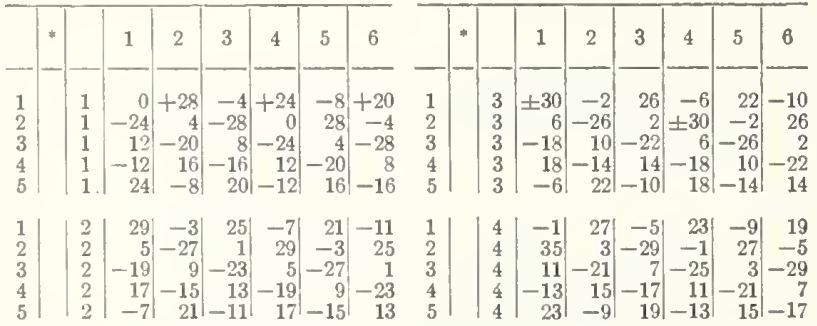

* The value of $b$ is 1 for integral degrees. Reducing both $a$ and $b$ by 1 leaving $c$ and $d$ constant reduces the displacement by $0.2^{\circ}$. Do not carry this process beyond a reduction of $0.8^{\circ}$

The designations of letters and the hole circle to which each applies are as follows:

\section{Letter Circle}

$a$ inner circle of guide ring

$b$ outer circle of guide ring

$c$ inner disk circle, i.e., disk nearest source oven

$d$ outer disk circle, i.e., disk nearest target.

Thus $a=1, b=1, c=3, d=2$ would mean that hole 1 of the inner guide circle is to be pinned to hole 3 of the inner disk circle, while hole 1 of the outer guide circle is to be pinned to hole 2 of the outer disk circle. From the table, one reads the displacement for this combination as $-2^{\circ}$. The table gives the values of $a, c$, and $d$ for integral numbers of degrees of relative advance. The value of $b$ is always 1 for integral numbers of degrees. The values of the advance on the chart may be reduced by multiples of $0.2^{\circ}$ up to and including a reduction of $0.8^{\circ}$ by reducing both $a$ and $b$ by one unit for each $0.2^{\circ}$ of reduction in angle, while leaving $c$ and $d$ unchanged. Since the designation of holes on the guide ring is 1,2 , $3,4,5,1,2, \ldots$ in an unending cycle, the value of $a$ or $b$ immediately below 1 is always 5 .

\subsection{Computation of the Initial Distribution Function}

The molecular density function $N_{0}(x, t)$ on a given target surface or $N_{\imath}(x, y)$ at a given time is the resultant of a series of elimination processes which have restricted the class of molecules which are permitted to enter the interaction space.
Within the molecular beam source, the molecular density is described by a density function of the three space coordinates, the three velocity components, and the time. Except for slight density deficiencies due to the constant loss of molecules through the source slit and their replacement by evaporation (or in the case of a permanent gas, by diffusion from a higher pressure source), the density is independent of position and time.

The velocity dependence is a normalized function of the form

$$
e^{-\frac{m}{2}\left(v_{s}^{2}+v_{v}^{2}+v_{s}^{2}\right)}
$$

Of all the molecules described by this density function, we want to know how many will be found in the area $d x d y$ or $d x d t$ encompassing a given space-time position $(x, y, t)$. We are not concerned with the normalization factors in the source density function, since we want only relative values of the final density $N_{0}$ or $N_{t}$ at different points.

In order for a molecule to have the coordinate $\left(x_{1}, y_{1}, z_{1}\right)$ at time $t_{1}$, the equations describing its motion must describe a line passing through the given time $t_{1}$. These may be written

$$
\begin{aligned}
& x=x_{1}+x^{\prime}\left(y-y_{1}\right) \\
& z=z_{1}+z^{\prime}\left(y-y_{1}\right) \\
& t=t_{1}+t^{\prime}\left(y-y_{1}\right),
\end{aligned}
$$

where $x^{\prime}=d x / d y, z^{\prime}=d z / d y$, and $t^{\prime}=d t / d y$.

The density function is then an integral in a four-dimensional space consisting of $z_{1}, x^{\prime}, z^{\prime}$, and $t^{\prime}$ of a density function in this space. This density function is easily determined from the density function describing the molecules within the source chamber. The limits of integration are, however, somewhat difficult to state in simple form. At the source opening $y=y_{s}$, the allowed region is the rectangular source opening. Substituting $y=y_{s}$ into eq (13.11) translates this condition into a rectangular allowed region in $x^{\prime}-z^{\prime}$ space. The position, but not the area of the rectangle, depends upon $z_{1}$. Similarly, the positions of the slit jaws, limiting $x$ and $z$ position as the particle goes through the collimator assembly, provide a second rectangular area in $x^{\prime}-z^{\prime}$ space. The allowed region is the region, if any, which is common to both rectangles. The chopper slits impose additional limitations on the allowed region for integration.

If time $t=0$ is chosen as the time when the axis of the second chopper slit is exactly alined with the collimator slit, the collimator slit allows passage at time $t$ of those molecules for which

$$
\left[z+r_{M}\right] \tan \omega t-\frac{a_{2}}{\cos \omega t}<x<\left[z+r_{M}\right] \tan \omega t+\frac{a_{2}}{\cos \omega t}
$$

where $\omega$ is the angular velocity of the chopper 
disk, $2 a_{2}$ is the width of the chopper slit, and $r_{M}$ is the radial distance from the chopper's axis of rotation to the plane $z=0$. A similar condition for passage exists in the plane of the first chopper disk with $\omega t+\theta$ replacing $\omega t$ and $a_{1}$ replacing $a_{2}$. The width of the first chopper slit is $2 a_{1}$, and the lead angle between the axis of the two chopper slits is $\theta$.

As in the work with the source and collimator, this inequality may be translated into relations in $x^{\prime}-z^{\prime}$ space by substituting the value of $y$ in the chopper slit plane into the inequalities of eq (13.12). In this case, however, the resulting relations depend upon the values of both $t^{\prime}$ and $z_{1}$, which are intended as variables of integration. The two sets of requirements in $x^{\prime}-z^{\prime}$ space form a diamond-shaped pattern when plotted. The area common to both the diamond and the rectangle (at fixed values of $z_{1}$ and $t^{\prime}$ ) represents the allowed range of coordinates in $x^{\prime}-z^{\prime}$ space for which a molecule can reach the desired point in space time without being intercepted by any aperture. As $z_{1}$ and $t^{\prime}$ are varied, the positions of the diamond and of the rectangle shift somewhat with relatively little change in volume. For sufficiently large or small values of either $z_{1}$ or $t^{\prime}$, the two quadrilaterals do not overlap at all.

The region in which an integration can be carried out is thus bounded by a closed figure in $z_{1}-t^{\prime}$ space. This region is subdivided into smaller regions characterized by differing topological properties. For example, in one region of $z_{1}-$

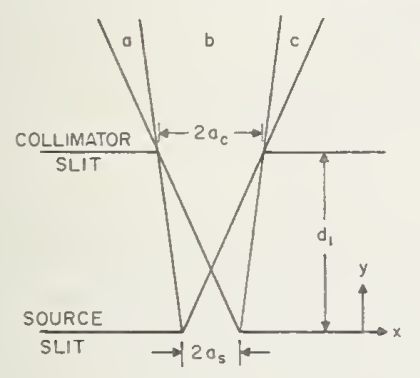

a

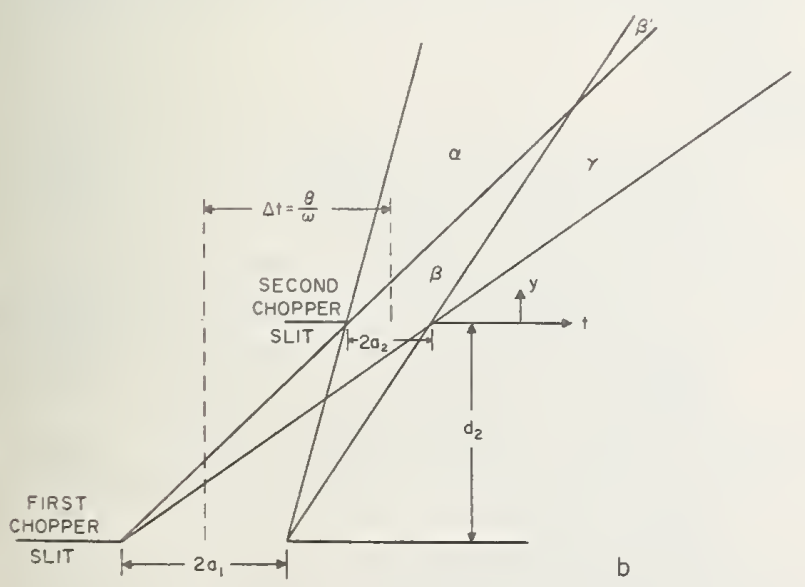

FIGURE 31 (a). Ray diagram for spatial collimation of the molecular beam. (b) Corresponding ray diagram in t-y space for chopping and monochromatizing the molecular beam. $t^{\prime}$ space, the region of overlap in $x^{\prime}-z^{\prime}$ space might be a triangle involving two sides of the diamond and one of the rectangle. In an adjoining $z_{1}-t^{\prime}$ region, the overlap in $x^{\prime}-z^{\prime}$ might be a quadrilateral involving the previous three sides and an adjacent side of the rectangle.

The various topological possibilities of overlap involving different combinations of sides of the two quadrilaterals thus constitute a series of boundary conditions of algebraically different form, although the equations defining the sides of the quadrilaterals are fairly simple. In perhaps a dozen or more regions of $z_{1}-t^{\prime}$ space, the boundaries of the allowed region in $x^{\prime}-z^{\prime}$ space can be stated as a group of straight line segments whose constants are certain functions of $z_{1}$ and $t^{\prime}$. In each region, then, it is possible to integrate first over $x^{\prime}-z^{\prime}$ space, with $z_{1}$ and $t^{\prime}$ carried as parameters and then over $z_{1}-t^{\prime}$ space, and the resulting quantities may be added. We have then computed the molecular density at the single point $\left(x_{1}, y_{1}, t_{1}\right)$.

In view of the extreme complication involved in calculating the molecular density at a given point, the prospect of computing an exact piecewise analytic density function $N_{o}(x, t)$ or $N_{t}(x, y)$ appears hopeless. The problem does lend itself to programmed automatic computation, however, and it is to be hoped that a practical computer program will be produced by future investigators.

\subsection{Simplified Approximate Computation of the Molecular Density Distribution}

Much of the complication in the preceding treatment of the formation of a molecular beam packet is due to the fact that the chopper blades do not remain parallel to the collimator slit axis as they cut the molecular beam. The effective cutting velocity as a function of position in the beam, is equal to $\omega\left(z+r_{M}\right)$. Since the slit widths are independent of $z$, this means that the transmission on time for each chopper is greater at points close to the chopper axis than at points near the rim. In our apparatus the limits of $z$ are $\pm 1.25 \mathrm{~cm}$, while $r_{M}$ is $4.375 \mathrm{~cm}$, leading to a variation in cutting velocities from $6 / 7$ to $8 / 7$ of the mean velocity.

To first order, the effects of a higher velocity for a positive value of $z$ are cancelled out by the lower velocity at the corresponding negative, value of $z$. Thus, if we neglect this effect altogether, we can expect an error of second order in $z / r_{M}$ or, in this experiment, an error of up to 5 percent. With this simplification, we become no longer concerned with coordinates and slopes in the $z$-direction. The integral in four-dimensional space in the last section thus reduces to one in two-dimensional $x^{\prime}-t^{\prime}$ space. Furthermore, the boundaries of the volume integral, which were extremely complicated in the last section, now reduce to a simple parallelogram in $x^{\prime}-t^{\prime}$ space.

An aid in finding the boundary conditions for the integration and one which also provides some physical insight is the pair of ray diagrams in figures $31 \mathrm{a}$ and $\mathrm{b}$. These figures are the projec- 
tions of limiting molecular paths in $x-y$ space and in $t-y$ space. In $x-y$ space the range of paths is limited by the source slit and the collimator slit. In $t-y$ space, the first and second chopper slits perform this function. Straight lines connecting the slit edges divide the accessible areas of $x-y$ space into regions $a, b$, and $c$ and divide $t-y$ space into regions $\alpha, \beta, \beta^{\prime}$, and $\gamma$. In finding the molecular density associated with the point $\left(x_{1}, y_{1}, t_{1}\right)$, we first note whether the given point lies in region $a, b$, or $c$.

If the point lies in region $b$, the limits of integration are

$$
\frac{x_{1}-a_{s}}{y_{1}+d_{1}}<x^{\prime}<\frac{x_{1}+a_{s}}{y_{1}+d_{1}}
$$

In region $a$, the upper limit is replaced by $\frac{x_{1}+a_{c}}{y_{1}}$; and in region $c$, the lower limit is replaced by $\frac{x_{1}-a_{c}}{y_{1}}$.
On the boundaries between regions, the alternate limits applying for the adjacent regions are equal. The diagram in $y-t$ space can be constructed only when the particle path in $x-y$ space is known. The $y$ planes of the two chopper slits are, of course, simply the positions of the disks. The widths of the slits in units of time are their geometrical widths divided by the mean cutting velocity $\omega r_{M}$. The center of each slit is displaced by the $x$ value of the particle path, as it crosses that slit plane, divided again by $\omega r_{M}$. An additional displacement $\theta / \omega$ is imposed upon the first chopper sit. Thus, we are able to construct figure $31 \mathrm{~b}$.

For a given point of observation $\left(t_{1}, y_{1}\right)$, the limits of integration in $t^{\prime}$ depend upon the region $\alpha, \beta, \beta^{\prime}$, or $\gamma$ in which the point lies. It is not difficult to show that the limits of integration for $t^{\prime}$ are as given in the following table:

Limits of integration in $\mathrm{t}^{\prime}$ as determined by location in figure $31 \mathrm{~b}$

\begin{tabular}{c|c|c}
\hline \hline Region & Lower limit of $t^{\prime}$ & Upper limit of $t^{\prime}$ \\
\hline$\alpha$ & $\frac{x_{1}-\left(y_{1}+d_{2}-y_{2}\right) x^{\prime}-a_{2}+r_{M} \theta}{\omega r_{M}\left(y_{1}+d_{2}-y_{2}\right)}$ & $\frac{x_{1}-\left(y_{1}-y_{2}\right) x^{\prime}+a_{1}}{\omega r_{M}\left(y_{1}-y_{2}\right)}$ \\
$\beta$ & $\frac{x_{1}-\left(y_{1}+d_{2}-y_{2}\right) x^{\prime}-a_{2}+r_{M} \theta}{\omega r_{M}\left(y_{1}+d_{2}-y_{2}\right)}$ & $\frac{x_{1}-\left(y_{1}+d_{2}-y_{2}\right) x^{\prime}+a_{2}+r_{M} \theta}{\omega r_{M}\left(y_{1}+d_{2}-y_{2}\right)}$ \\
$\beta^{\prime}$ & $\frac{x_{1}-\left(y_{1}-y_{2}\right) x^{\prime}-a_{1}}{\omega r_{M}\left(y_{1}-y_{2}\right)}$ & $\frac{x_{1}-\left(y_{1}-y_{2}\right) x^{\prime}+a_{1}}{\omega r_{M}\left(y_{1}-y_{2}\right)}$ \\
$\gamma$ & $\frac{x_{1}-\left(y_{1}-y_{2}\right) x^{\prime}-a_{1}}{\omega r_{M}\left(y_{1}-y_{2}\right)}$ & $\frac{x_{1}-\left(y_{1}+d_{2}-y_{2}\right) x^{\prime}+a_{2}+r_{M} \theta}{\omega r_{M}\left(y_{1}+d_{2}-y_{2}\right)}$ \\
\hline
\end{tabular}

The location of the point under consideration (in space $a, b$, or $c$, and $\alpha, \beta, \beta^{\prime}$, or $\gamma$ ) is determined by its coordinates $\left(x_{1}, y_{1}, t_{1}\right)$ and the parameters of the experiment. When an entire distribution, $N_{t}\left(x_{1}, y_{1}\right)$ or $N_{y}\left(x_{1}, t_{1}\right)$, is to be mapped by integrations at a series of points, some points will be found in all three of the areas in $x-y$ space and in at least three of the four areas in $t-y$ space. Thus, the expression for $N$ is piecewise-analytic in its dependent variables, and separate expressions must be written for each of the nine or more analytic segments.

\section{References}

[1] R. G. J. Fraser, Molecular Rays (Cambridge Univ. Press, Cambridge, England, 1931).

[2] R. G. J. Fraser, Molecular Beams (Methuen \& Co., Ltd., London, England, 1937).

[3] M. Knudsen, Ann. Physik 28, 76, 999 (1909); 29, 179 (1909); and 48, 1113 (1915).

[4] E. H. Kennard, Kinetic Theory of Gases (McGrawHill Book Co., Inc., New York, 1938).

[5] M. Inudsen, Kinetic Theory of Gases (Methuen \& Co., Ltd., London, England, 1934).

[6] S. T'exler, Rev. Mod. Phys. 30, 402 (1958).

[7] J. E. Lennard-Jones and A. F. Devonshire, Nature 137, 1069 (1936); Proc. Roy. Soc. (London) A156, $6(1935)$.

[8] J. E. Lennard-Jones and C. Strachan, Proc. Roy. Soc. (London) A150, 442, 456 (1935).

[9] S. A. Schaaf, Discussion at Symposium on Rarefied Gas Dynamics, Berkeley, California (1960).

[10] S. Nocilla, Rarefied Gas Dynamics, p. 169 (Academic Press, Inc., New York, 1960).
[11] H. Oguchi, Rarefied Gas Dynamics, p. 501 (Academic Press, Inc., New York, 1960)

[12] J. Statder, G. Goodwin and M. O. Creuyer, NACA Report \# 1052 (1951).

[13] F. S. Sherman, NACA Tech. Note 3298 (1955).

[14] M. V. Morkoven, "Fluctuations and Hot wire Anemometry in Compressible Flows," Wind Tunnel Agardograph Series, NATO Advisory Group for Aeronautical Research and Development, Paris (1956).

[15] J. Winkler, Rev. Sci. Instr. 19, 307 (1948).

[16] H. Schardin, "Toepler's Schlieren Method," Translation 156, David W. Taylor Model Basin, U.S. Navy (1947).

[17] L. Dunoyer, Compt. Rend. 152, 594 (1911); J. Phys. Radium 8, 142 (1911).

[18] S. Estermann and O. Stern, Z. physik. Chem. 106, 399, 403 (1923).

[19] W. Gerlach and O. Stern, Ann. Physik 74, 673 (1924) and $\boldsymbol{7 6}_{6}, 163$ (1925).

[20] K. F. Smith, Nature 167, 942 (1951). 
[21] V. W. Cohen, D. A. Gilbert, S. Wexler, and I. S. Goodman, Phys. Rev. 95, 569, 570 (1954).

[22] J. B. Taylor, Phys. Rev. 35, 375 (1930).

[23] E. Langmuir and K. H. Kingdon, Proc. Roy. Soc. (London) A10\%, 61 (1925).

[24] G. Wessel and H. Lew, Phys. Rev. 92, 641 (1953).

[25] E. Winkler, U.S. Naval Ordnance Lab., White Oak, Md., Memo 10118 (1949).

[26] J. Dimeff, R. K. Hallett and C. F. Hansen, NASA Tech. Report TN-2845 (1952).

[27] W. B. Kunkel and F. C. Hurlbut, J. Appl. Phys. 28, 827 (1957).

[28] F. C. Hurlbut, "An Electron Beam Density Probe for Measurements in Rarefied Gas Flows," WADC TR-57-644 (1957).

[29] H. Boersch, Z. Physik 10\%, 493 (1937).

[30] L. Marton, J. Appl. Phys. 19, 687 (1948).

[31] L. Marton and S. H. Lachenbruch, J. Appl. Phys. 20, 1171 (1949).

[32] D. L. Reverdin, J. Appl. Phys. 22, 257 (1951).

[33] F. Ollendorff, Elektronik Freier Raumladungen Springer-Verlag, Vienna, (1951)

[34] I. Estermann, Rev. Mod. Phys. 18, 300 (1946).

[35] K. R. Spangenberg, Vaccum Tubes (McGraw-Hill Book Co., Inc., New York, 1948).

[36] E. Everhart, G. Stone and R. J. Carbone, Phys. Rev. 99, 1287 (1955).

[37] H. Bruck and M. Bricka, Ann. Radioelect. 3, 339 (1948).
[38] G. Liebmann and E. M. Grad, Proc. Roy. Soc. (London) B64, 1956 (1951).

[39] I. K. Zworykin, G. A. Mortin, E. G. Ramberg, J. Hillier, and A. W. Vance, Electron Optics and the Electron Microscope (John Wiley \& Sons, Inc., New York, 1945).

[40] W. Glaser, Elektronen-Optik (Springer-Verlag, Vienna, 1952).

[41] A. Sommerfeld, Electrodynamics (Academic Press, Inc., New York, 1952).

[42] N. F. Ramsey, Molecular Beams (Oxford University Press, London, England, 1956).

[43] J. G. King and J. R. Zacharias, Advances in Electronics and Electron Physics 8, 1 (Academic Press, Inc., New York, 1956).

[44] A. I. Reimann, Vacuum Technique (Chapman \& Hall, Ltd., London, England, 1952).

[45] L. S. Ornstein, W. J. H. Moll and H. C. Burger, Objektive Spectralphotometrie (F. Vieweg \& Sohn, Braunschweig, Germany, 1932).

[46] A. I. Bennett, Jr., and I. Estermann, "Distribution of Velocities in Molecular Beams of Potassium," Carnegie Institute of Technology, Tech. Report \#4 (1953).

[47] R. V. Churchill, Modern Operational Mathematics in Engineering (McGraw-Hill Book Co., Inc., New York, 1944). 




\section{THE NATIONAL BUREAU OF STANDARDS}

The scope of activities of the National Bureau of Standards at its major laboratories in Washington, D.C., and Boulder, Colorado, is suggested in the following listing of the divisions and sections cngaged in technical work. In general, each section carries out spccialized research, development, and engineering in the field indicatcd by its title. A brief description of the activities, and of the resultant publications, appears on the inside of the front cover.

\section{WASHINGTON, D.C.}

Electricity. Resistance and Reactance. Electrochemistry. Electrical Instrumcnts. Magnetic Measurements. Dielectrics. High Voltage. Absolute Electrical Measurements.

Metrology. Photometry and Colorimetry. Refractometry. Photographic Research. Length. Engineering Metrology. Mass and Scale. Volumetry and Densimetry.

Heat. Temperature Physics. Heat Measurcments. Cryogenic Physics. Equation of State. Statistical Physics. Radiation Physics. X-ray. Radioactivity. Radiation Theory. High Energy Radiation. Radiological Equipment. Nucleonic Instrumentation. Neutron Physics.

Analytical and Inorganic Chemistry. Pure Substances. Spectrochemistry. Solution Chemistry. Standard Reference Matcrials. Applied Analytical Research. Crystal Chemistry.

Mechanics. Sound. Pressure and Vacuum. Fluid Mechanics. Engineering Mechanics. Rheology. Combustion Controls.

Polymers. Macromolecules: Synthesis and Structure. Polymer Chemistry. Polymer Physics. Polymer Characterization. Polymer Evaluation and Testing. Applicd Polymer Standards and Research. Dental Rcscarch.

Metallurgy. Engineering Metallurgy. Microscopy and Diffraction. Metal Reactions. Metal Physics. Flectrolysis and Meial Deposition.

Inorganic Solids. Engineering Ceramics. Glass. Solid State Chemistry. Crystal Growth. Physical Properties. Crystallography.

Building Research. Structural Enginecring. Fire Rescarch. Mechanical Systems. Organic Building Materials. Codes and Safety Standards. Heat Transfer. Inorganic Building Materials. Metallic Building Materials.

Applied Mathematics. Numerical Analysis. Computation. Statistical Engineering. Mathematical Physics. Operations Research.

Data Processing Systems. Components and Techniques. Computer Technology. Measurements Automation. Engineering Applications. Systems Analysis.

Atomic Physics. Spectroscopy. Infrared Spcetroscopy. Far Ultraviolet Physics. Solid State Physics. Electron Physics. Atomic Physics. Plasma Spectroscopy.

Instrumentation. Engineering Electronics. Electron Devices. Electronic Instrumentation. Mechanical Instruments. Basic Instrumentation.

Physical Chemistry. Thermochemistry. Surface Chemistry. Organic Chemistry. Molecular Spcctroscopy. Elementary Processes. Mass Spectrometry. Photochemistry and Radiation Chemistry.

Office of Weights and Measures.

BOULDER, COLO.

Cryogenic Engineering Laboratory. Cryogenic Equipment. Cryogenic Processes. Cryogenic Properties of Solids. Cryogenic Technical Services. Properties of Cryogenic Fluids.

\section{CENTRAL RADIO PROPAGATION LABORATORY}

Ionosphere Research and Propagation. Low Frequency and Very Low Frequency Rescarch. Ionosphere Research. Prediction Services. Sun-Earth Relationships. Field Engincering. Radio Warning Scrvices. Vertical Soundings Research.

Radio Propagation Engineering. Data Reduction Instrumentation. Radio Noisc. Tropospheric Mcasurements. Tropospheric Analysis. Propagation-Terrain Effects. Radio-Meteorology. Lower Atmosphere Physics.

Radio Systems. Applied Electromagnetic Theory. High Frequency and Very High Frequency Research. Frequency Utilization. Modulation Research. Antenna Research. Radiodetermination.

Upper Atmosphere and Space Physics. Upper Atmosphere and Plasma Physics. High Latitude Ionospherc Physics. Ionosphere and Exosphere Scatter. Airglow and Aurora. Ionospheric Radio Astronomy.

\section{RADIO STANDARDS LABORATORY}

Radio Physics. Radio Broadcast Service. Radio and Microwave Materials. Atomic Frequency and Time-Interval Standards. Radio Plasma. Millimeter-Wave Rescarch.

Circuit Standards. High Frequency Electrical Standards. High Frequency Calibration Services. High Frequency Impedance Standards. Microwave Calibration Services. Microwave Circuit Standards. Low Frequency Calibration Services. 
